Supporting Information for

\title{
Thiostrepton Variants Containing a Contracted Quinaldic Acid Macrocycle Result from Mutagenesis of the Second Residue
}

\author{
Feifei Zhang, Chaoxuan Li and Wendy L. Kelly*
}

Table of Contents

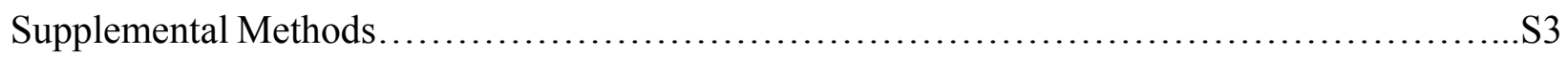

Figure S1. Two diagnostic daughter ions from thiostrepton MALDI MS/MS..................S5

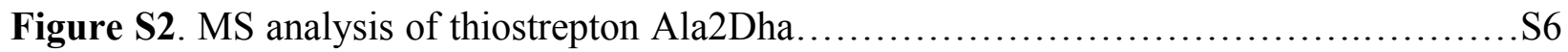

Figure S3. Structure and numbering system used for thiostrepton Ala2Dha................. S8

Figure S4. ${ }^{1} \mathrm{H}$ NMR spectrum of thiostrepton Ala2Dha..................................... 9

Figure S5. ${ }^{13} \mathrm{C}$ NMR spectrum of thiostrepton Ala2Dha.................................. 10

Figure S6. DEPT-135 spectrum of thiostrepton Ala2Dha.............................. S11

Figure S7. gHSQC spectrum of thiostrepton Ala2Dha......................................

Figure S8. gCOSY spectrum of thiostrepton Ala2Dha...................................... 13

Figure S9. gHMBC spectrum of thiostrepton Ala2Dha................................ 14

Table S1. ${ }^{1} \mathrm{H}$ and ${ }^{13} \mathrm{C}$ NMR assignments of thiostrepton Ala2Dha........................... 15

Figure S10. MS analysis of thiostrepton Ala2Dhb..................................... 17

Figure S11. Structure and numbering system used for thiostrepton Ala2Dhb ..................S19

Figure S12. ${ }^{1} \mathrm{H}$ NMR spectrum of thiostrepton Ala2Dhb.................................S20

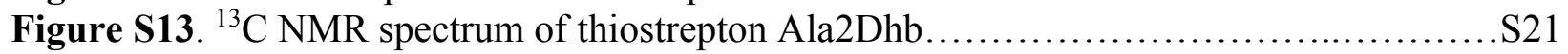

Figure S14. DEPT-135 spectrum of thiostrepton Ala2Dhb ..........................S22

Figure S15. gHSQC spectrum of thiostrepton Ala2Dhb .............................. 23

Figure S16. gCOSY spectrum of thiostrepton Ala2Dhb .............................. S24

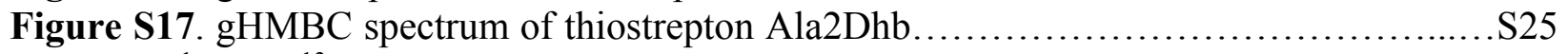

Table S2. ${ }^{1} \mathrm{H}$ and ${ }^{13} \mathrm{C}$ NMR assignments of thiostrepton Ala2Dhb.......................S26

Figure S18. MS analysis of thiostrepton Ala2Ile- $\Delta$ Ile1 ................................S28

Figure S19. Structure and numbering system used for thiostrepton Ala2Ile- $\Delta$ Ile $1 \ldots \ldots \ldots \ldots . .$. S30

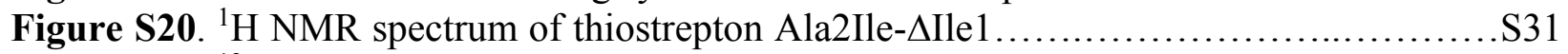

Figure S21. ${ }^{13} \mathrm{C}$ NMR spectrum of thiostrepton Ala2Ile- $\Delta$ Ile1 ............................S32

Figure S22. DEPT-135 spectrum of thiostrepton Ala2Ile- $\Delta$ Ile1 ...........................S33

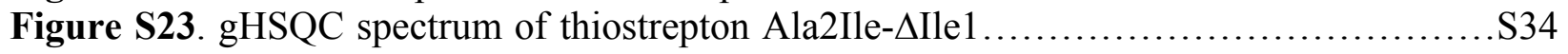

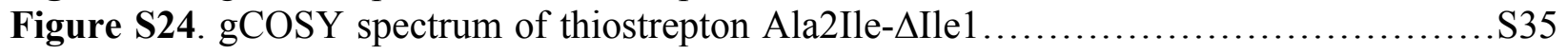

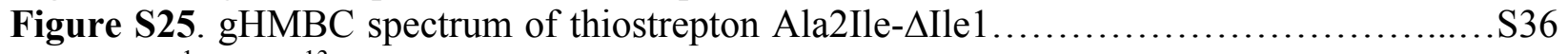

Table S3. ${ }^{1} \mathrm{H}$ and ${ }^{13} \mathrm{C}$ NMR assignments of thiostrepton Ala2Ile- $\Delta$ Ile1 ......................S37

Figure S26. MS analysis of thiostrepton Ala2Met...................................... 39

Figure S27. MS analysis of thiostrepton Ala2Phe........................................ 44

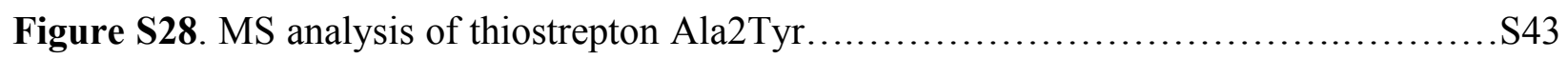


Figure S29. MS analysis of thiostrepton Ala2Val-Ile1 ................................. 45

Figure S30. Structure and numbering system used for thiostrepton Ala2Val- $\Delta$ Ile1 ...........S47

Figure S31. ${ }^{1} \mathrm{H}$ NMR spectrum of thiostrepton Ala2Val- $\Delta$ Ile1 ...........................S48

Figure S32. ${ }^{13} \mathrm{C}$ NMR spectrum of thiostrepton Ala2Val- $\Delta \mathrm{Ile} 1 \ldots \ldots \ldots \ldots \ldots \ldots \ldots \ldots \ldots \ldots . . . \ldots \ldots$

Figure S33. DEPT-135 spectrum of thiostrepton Ala2Val- $\Delta$ Ile1 $\ldots \ldots \ldots \ldots \ldots \ldots \ldots \ldots \ldots \ldots . . . . . \ldots 50$

Figure S34. gHSQC spectrum of thiostrepton Ala2Val- $\Delta$ Ile1 $\ldots \ldots \ldots \ldots \ldots \ldots \ldots \ldots \ldots \ldots \ldots$. 51

Figure S35. gCOSY spectrum of thiostrepton Ala2Val- $\Delta$ Ile1 $\ldots \ldots \ldots \ldots \ldots \ldots \ldots \ldots \ldots \ldots \ldots . . . \ldots \ldots 2$

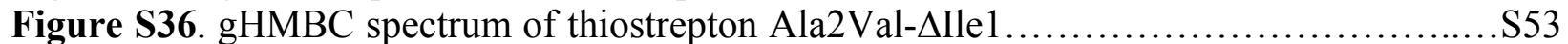

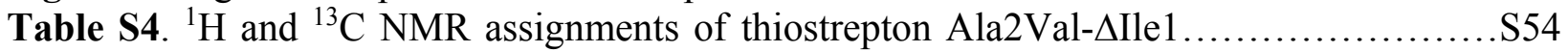

Figure S37. HPLC-MS analysis of a crude culture extract from S. laurentii NDS1/intA2L ..................................................................... 56

Figure S38. HPLC-MS analysis of a crude culture extract from S. laurentii NDS1/int-A2M....S58

Figure S39. HPLC-MS analysis of a crude culture extract from S. laurentii NDS1/int-A2F.....S60

Figure S40. A representative MS analysis revealing the presence of shunt metabolites of thiostrepton biosynthesis.............................................. 62

Table S5. Summary of HR-MALDI-MS results of thiostrepton Ala2 analogues...............S65

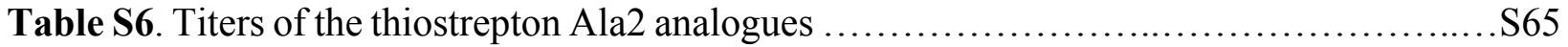

Table S7. Aqueous solubilities of thiostrepton Ala2 analogues............................S66

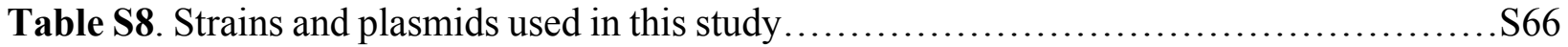

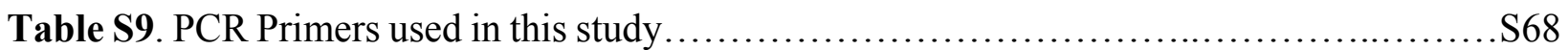

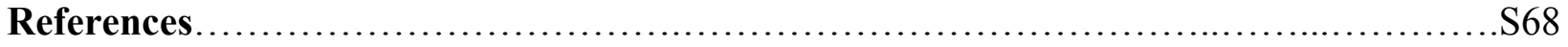




\section{Supplemental Methods}

General Materials and Methods. Unless otherwise indicated, all chemicals and solvents were purchased from common vendors and used as received. Enzymes and buffers were acquired from New England Biolabs. The Wizard ${ }^{\circledR}$ Genomic DNA Purification Kit was used to isolate Streptomyces genomic DNA. Isolation of plasmids and fosmids from E. coli strains were performed using the QIAprep Spin Miniprep Kit and DirectPrep 96 MiniPrep Kit from Qiagen. Polymerase chain reaction (PCR) products were cloned using the StrataClone Blunt PCR Cloning Kit (Agilent Technologies). 96-well tissue culture plates and 96-well black with clear flat bottom plates were obtained from Becton Dickinson and Corning, respectively. 384-well black plates were purchased from PerkinElmer. A BioTek H4 Multi-Mode Microplate Reader was used for optical density, fluorescence, and luminescence measurements. All oligonucleotides were synthesized by Integrated DNA Technologies. DNA sequencing was performed by Eurofins MWG Operon, and sequence analysis was completed using the VectorNTI software package from Invitrogen.

High performance liquid chromatography (HPLC) analysis was performed on a Beckman Coulter System Gold instrument. High-resolution matrix-assisted laser desorption/ionization mass spectrometry (HR-MALDI-MS), MALDI-MS/MS and HPLC-MS were conducted with an Applied Biosystems 4700 Proteomics Analyzer and a Micromass Quattro LC at the Georgia Institute of Technology Bioanalytical Mass Spectrometry Facility. High-resolution electrospray ionization mass spectrometry (HR-ESI-MS) was performed on a Thermo LTQ-FTMS at the Emory University Mass Spectrometry Center. For HPLC-MS, a Phenomenex Synergi RP column $(250 \mathrm{~mm} \times 2 \mathrm{~mm}, 4 \mu \mathrm{m})$ (Torrance, CA) was developed at $0.25 \mathrm{~mL} \mathrm{~min}^{-1}$ with $20 \%$ solvent B in solvent A for 8 min followed by a gradient from 20 to $100 \%$ solvent B over 35 min (Solvent A: $5 \%$ acetonitrile and $0.1 \%$ formic acid in water; solvent B: $5 \%$ water and $0.1 \%$ formic acid in acetonitrile). NMR experiments were completed on a Bruker $500 \mathrm{MHz}$ spectrometer according to standard pulse sequences supplied with the instrument at the Georgia Institute of Technology School of Chemistry and Biochemistry.

Bacterial Strains, Plasmids, and Growth Media. Streptomyces laurentii ATCC 31255 (S. laurentii), methicillin-resistant Staphylococcus aureus ATCC 10537 (MRSA), vancomycinresistant Enterococcus faecium ATCC 12952 (VRE), Bacillus sp. ATCC 27859 (Bacillus), and Escherichia coli (E. coli) ATCC 27856 were all purchased from American Type Culture Collection (ATCC). E. coli EPI300 was obtained from Epicentre ${ }^{\circledR}$. All strains, plasmids, and fosmids are itemized in Table S8, and primers are listed in Table S9. All E. coli strains were grown in Luria-Bertani liquid or solid medium with the appropriate antibiotic(s). For the selective growth of $E$. coli or Streptomyces, the following antibiotics and concentrations were used: apramycin (50 $\left.\mu \mathrm{g} \mathrm{mL} L^{-1}\right)$, kanamycin $\left(50 \mu \mathrm{g} \mathrm{mL}^{-1}\right)$, ampicillin $\left(100 \mu \mathrm{g} \mathrm{mL}^{-1}\right)$, nalidixic acid $\left(25 \mu \mathrm{g} \mathrm{mL}^{-1}\right)$, and chloramphenicol $\left(12.5 \mu \mathrm{g} \mathrm{mL}^{-1}\right)$. MS agar and ISP3 agar were used for the intergeneric conjugation of S. laurentii NDS1 and sporulation of S. laurentii strains, respectively. ${ }^{1,2}$

Purification and Mass Spectrometry Analyses of Thiostrepton Ala2 Analogues. S. laurentii variant culture extracts were first precipitated as previously described. ${ }^{3}$ The solid residue of the culture extract was dissolved in a minimum volume of chloroform and mixed with 10 volumes of $n$-hexane followed by centrifugation for $5 \mathrm{~min}$ at $4000 \mathrm{rpm}(2559 \times \mathrm{g})$ and $25^{\circ} \mathrm{C}$. The precipitate was then dissolved in a minimum volume of dichloromethane:ethanol (4:1). Diethyl ether (5 volumes) was added to the sample and the mixture was centrifuged at $4000 \mathrm{rpm}$ ( $2559 \mathrm{x}$ 
g) for $5 \mathrm{~min}$ at $25^{\circ} \mathrm{C}$. The solid residue was dissolved in chloroform:methanol (4:1) and further purified by semi-preparative HPLC using a Phenomenex Luna C18(2) column (250 x $10 \mathrm{~mm}, 5$ $\mu \mathrm{m})$ with water and acetonitrile, as described below, at $4.3 \mathrm{~mL} \mathrm{~min}^{-1}$ while monitoring absorbance at $254 \mathrm{~nm}$.

Purification of thiostreptons Ala2Dha, Ala2Dhb, Ala2Met, Ala2Phe, Ala2Tyr, and Ala2Ile- $\Delta$ Ile1, and Ala2Val- $\Delta$ Ile1 all utilized the same gradient: acetonitrile was increased from 0 to $100 \%$ in water over 30 min. Thiostrepton Ala2Ile- $\Delta$ Ile1 and Ala2Val- $\Delta$ Ile1 were subjected to a second round of semi-preparative HPLC purification by holding the mobile phase constant at $40 \%$ acetonitrile in water for $10 \mathrm{~min}$. Purified samples were analyzed by HPLC-MS, MALDI-MS/MS, either HR-ESI-MS or HR-MALDI-MS, and stored under argon at $-80^{\circ} \mathrm{C}$. Thiostreptons Ala2Dha, Ala2Dhb, Ala2Ile- $\Delta$ Ile1, and Ala2Val- $\Delta$ Ile1 were further analyzed by one- and two-dimensional NMR.

Kinetic solubilities of the thiostreptons were determined using a previously described protocol. $^{4,5}$ 
Figure S1. Two diagnostic daughter ions from the MALDI MS/MS of thiostrepton A and its analogues used to confirm the identity of the second residue. Fragment $\mathbf{A}$ corresponds to the loss of the quinaldic acid (QA) moiety and Ile1 from the parent ion, and fragment $\mathbf{B}$ additionally lacks the second residue $(\mathrm{Xxx} 2)$.

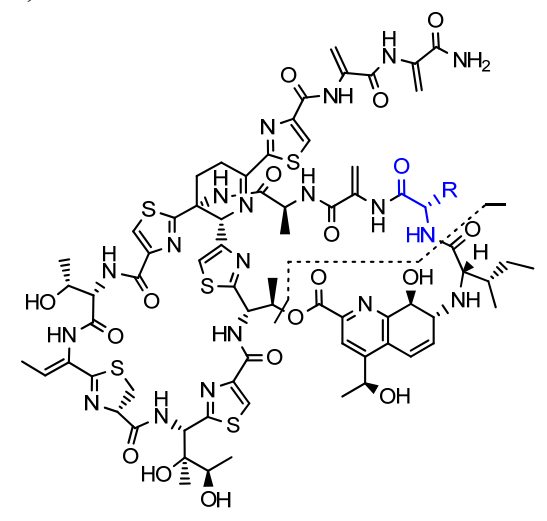

Parent lon: $[\mathrm{M}+\mathrm{H}]^{+}$
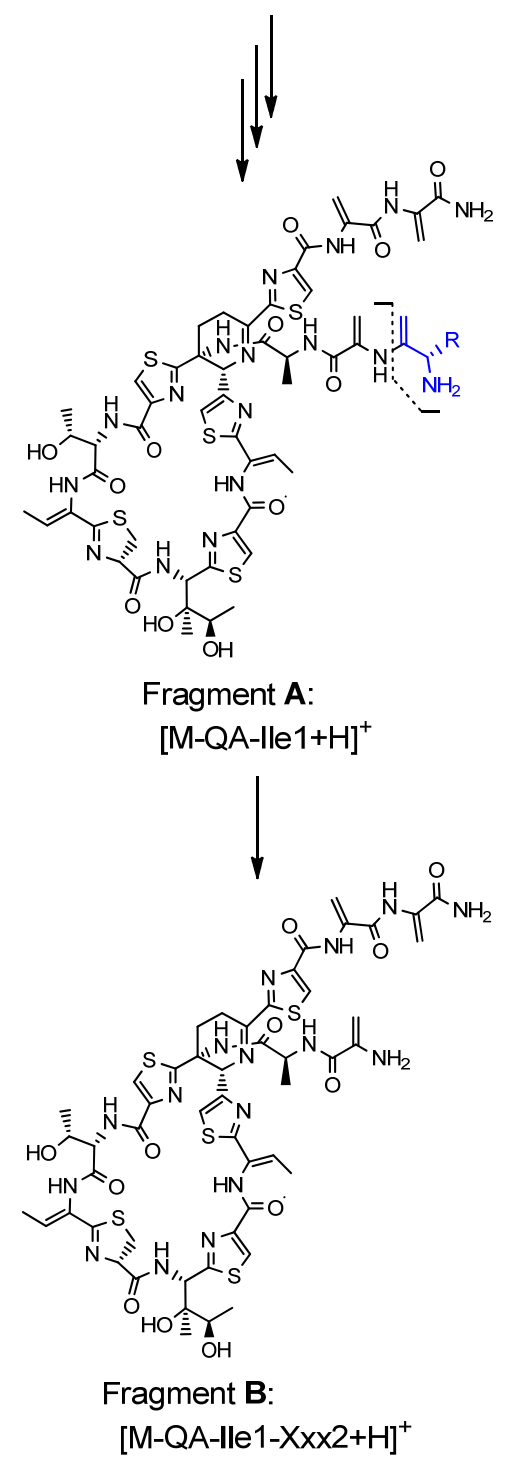
Figure S2. MS analysis of thiostrepton Ala2Dha isolated from S. laurentii NDS1/int-A2S.

(A) HPLC-MS analysis. (1) Chromatogram extracted for $\mathrm{m} / \mathrm{z} 831.7$, the calculated $[\mathrm{M}+2 \mathrm{H}]^{2+}$ ion of thiostrepton Ala2Dha. (2) Total ion chromatogram.

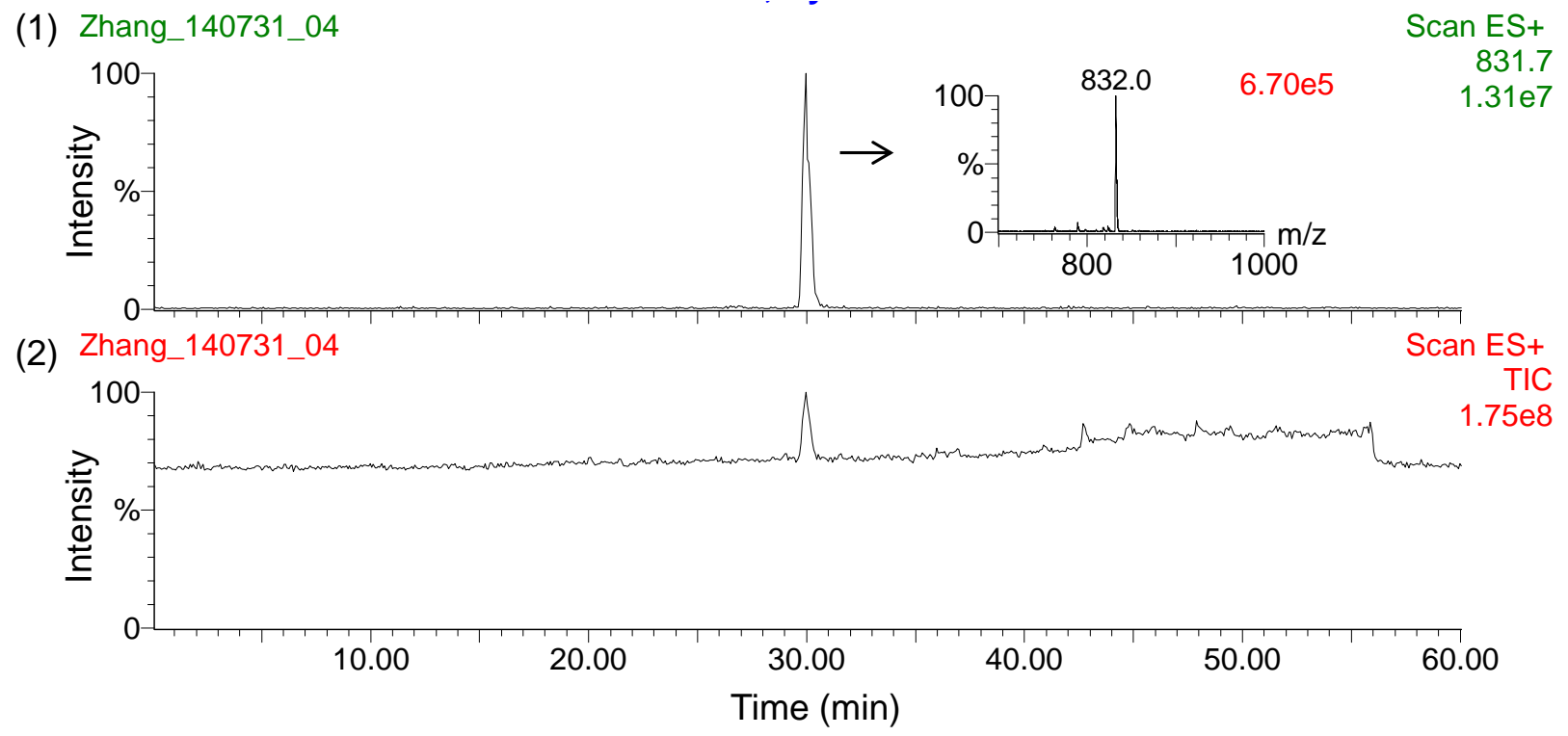

(B) MALDI MS spectrum of thiostrepton Ala2Dha.

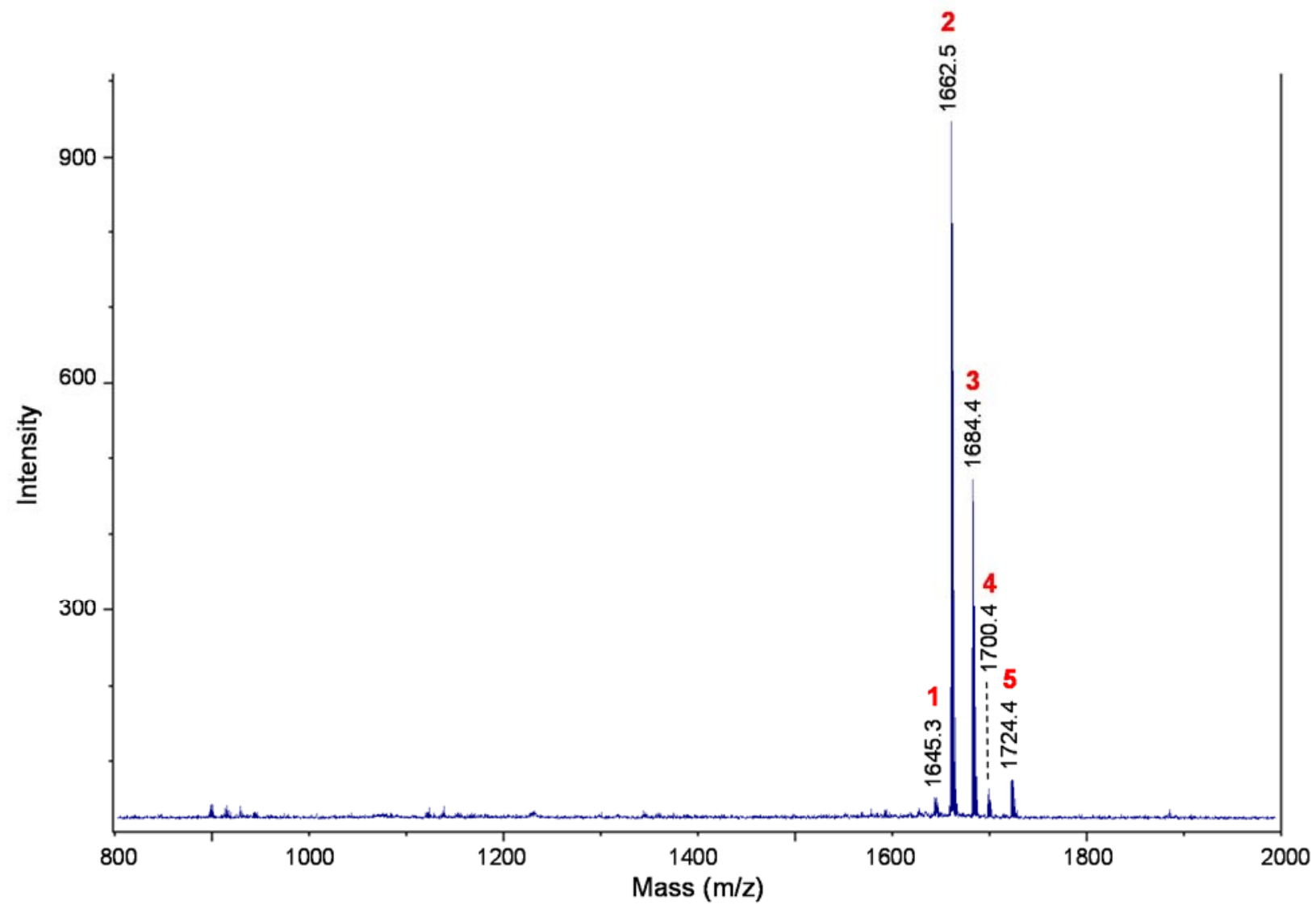


(C) MALDI MS/MS of parent ion $\mathrm{m} / \mathrm{z} 1662.5$.

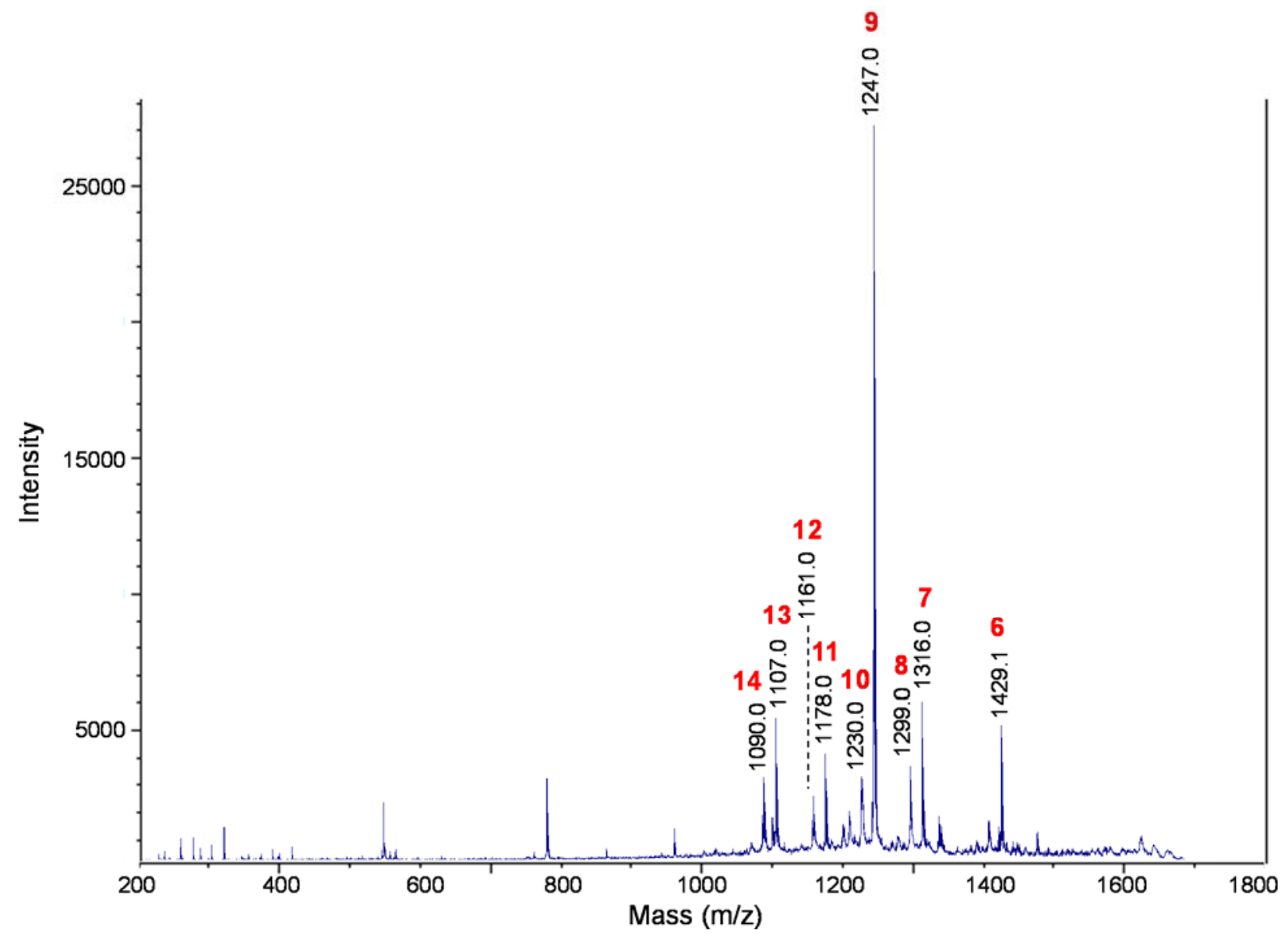

(D) Table and structure showing key ions and fragments in the MALDI MS and MS/MS of thiostrepton Ala2Dha.

\begin{tabular}{|c|c|c|}
\hline Fragment & Expected & Observed \\
\hline 1. $\mathrm{M}-\mathrm{OH}+\mathrm{H}^{+}$ & 1645.5 & 1645.3 \\
\hline 2. $\mathrm{M}+\mathrm{H}^{+}$(Parent ion) & 1662.5 & 1662.5 \\
\hline 3. $\mathrm{M}+\mathrm{Na}^{+}$ & 1684.5 & 1684.4 \\
\hline 4. $\mathrm{M}+\mathrm{K}^{+}$ & 1700.4 & 1700.4 \\
\hline 5. $\mathrm{M}+\mathrm{Cu}^{+}$ & 1724.4 & 1724.4 \\
\hline 6. $\mathrm{M}-\mathrm{QA}+\mathrm{H}^{+}$ & 1429.4 & 1429.1 \\
\hline 7. M-QA-Ile1+H ${ }^{+}$ & 1316.3 & 1316.0 \\
\hline 8. M-QA-Ile1-OH+H+ & 1299.3 & 1299.0 \\
\hline 9. M-QA-Ile1-Dha2+H+ & 1247.3 & 1247.0 \\
\hline 10. M-QA-Ile1-Dha2-OH+H+ & 1230.3 & 1230.0 \\
\hline 11. M-QA-Ile1-Dha2-Dha3+H+ ${ }^{+}$ & 1178.3 & 1178.0 \\
\hline 12. M-QA-Ile1-Dha2-Dha3-OH+H+ & 1161.3 & 1161.0 \\
\hline 13. M-QA-Ile1-Dha2-Dha3-Ala4+H+ & 1107.3 & 1107.0 \\
\hline 14. M-QA-Ile1-Dha2-Dha3-Ala4-OH+H+ & 1090.2 & 1090.0 \\
\hline
\end{tabular}

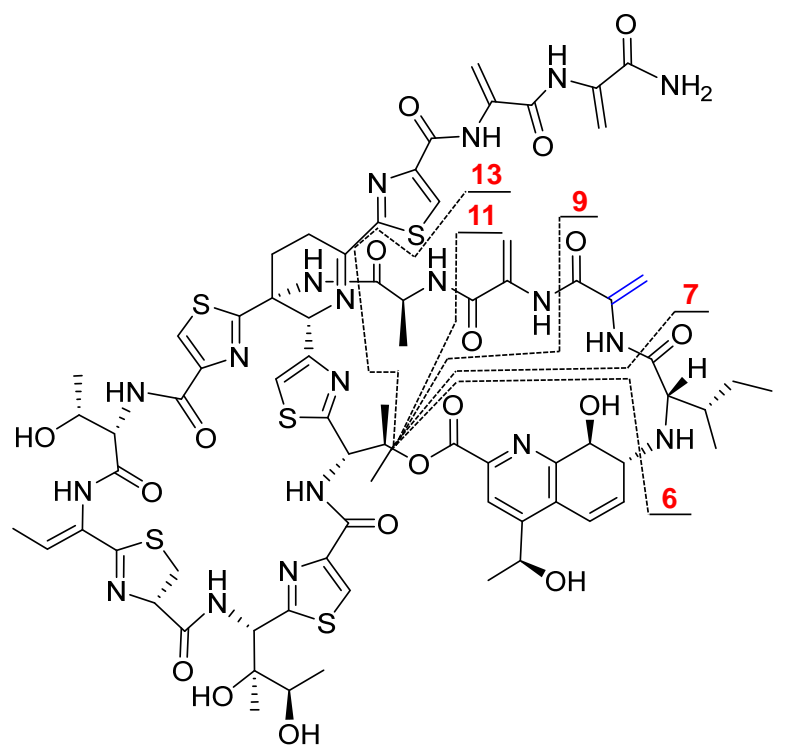


Figure S3. Structure and numbering system used for thiostrepton Ala2Dha.

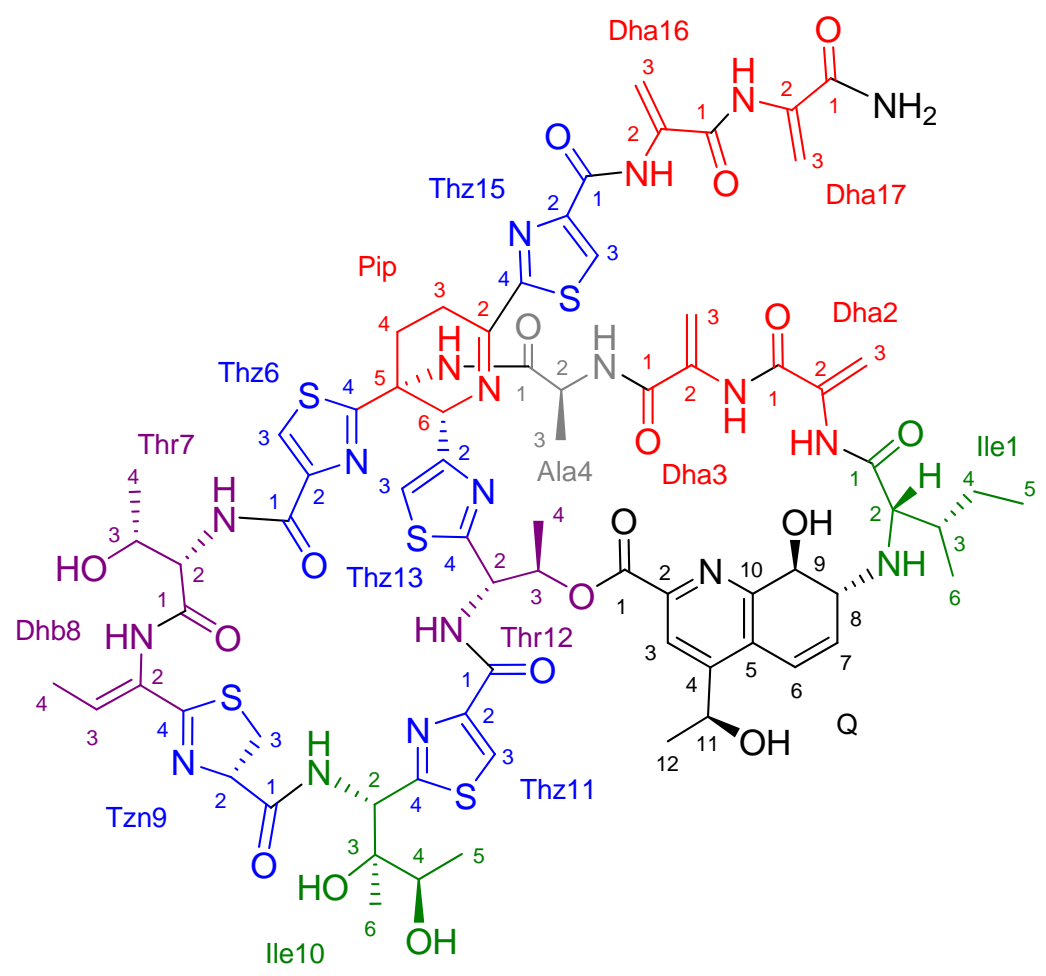




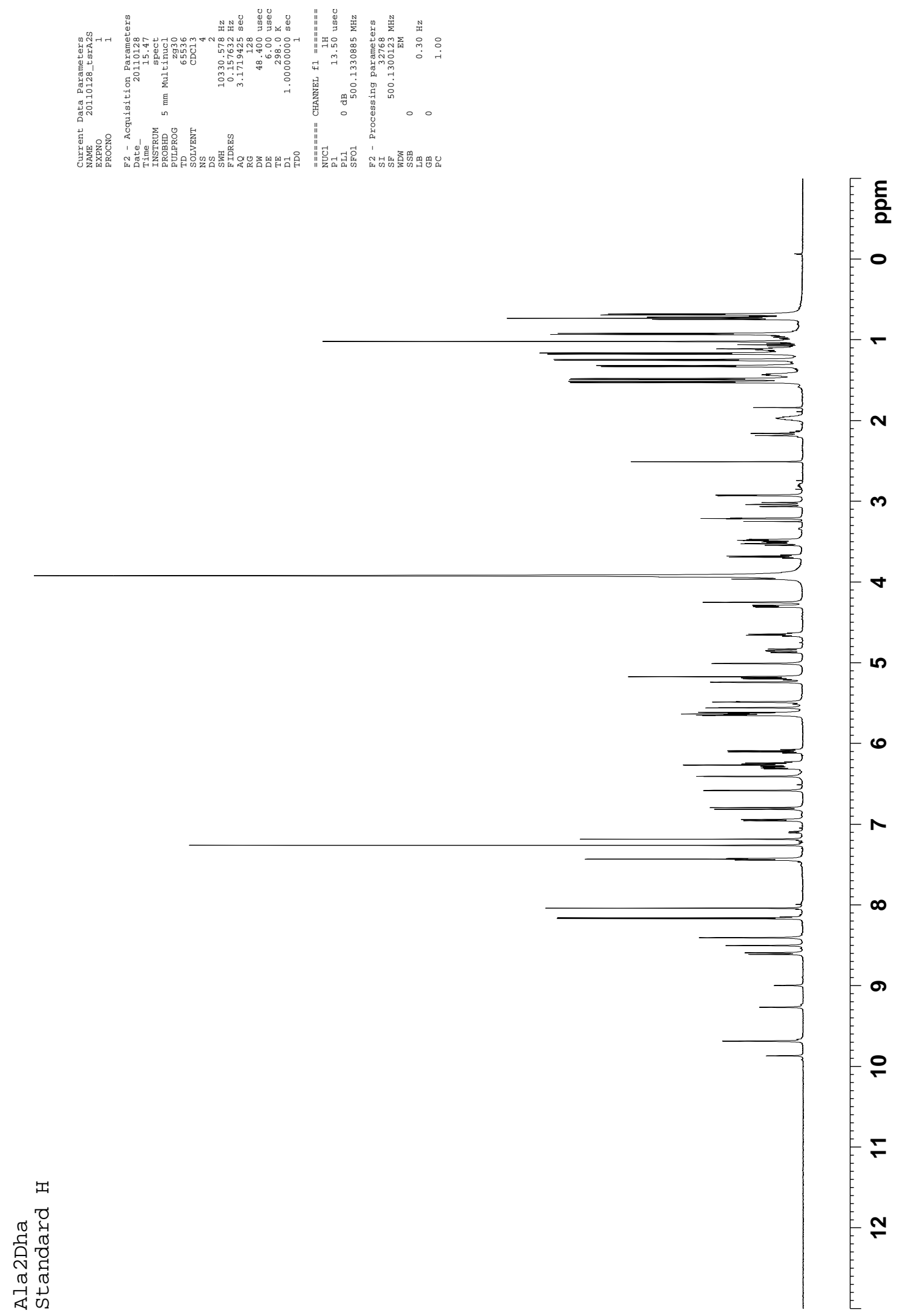

Figure S4. ${ }^{1} \mathrm{H}$ NMR spectrum of thiostrepton Ala2Dha $\left(500 \mathrm{MHz}, \mathrm{CDCl}_{3}-\mathrm{CD}_{3} \mathrm{OD} 4: 1,25{ }^{\circ} \mathrm{C}\right)$. 


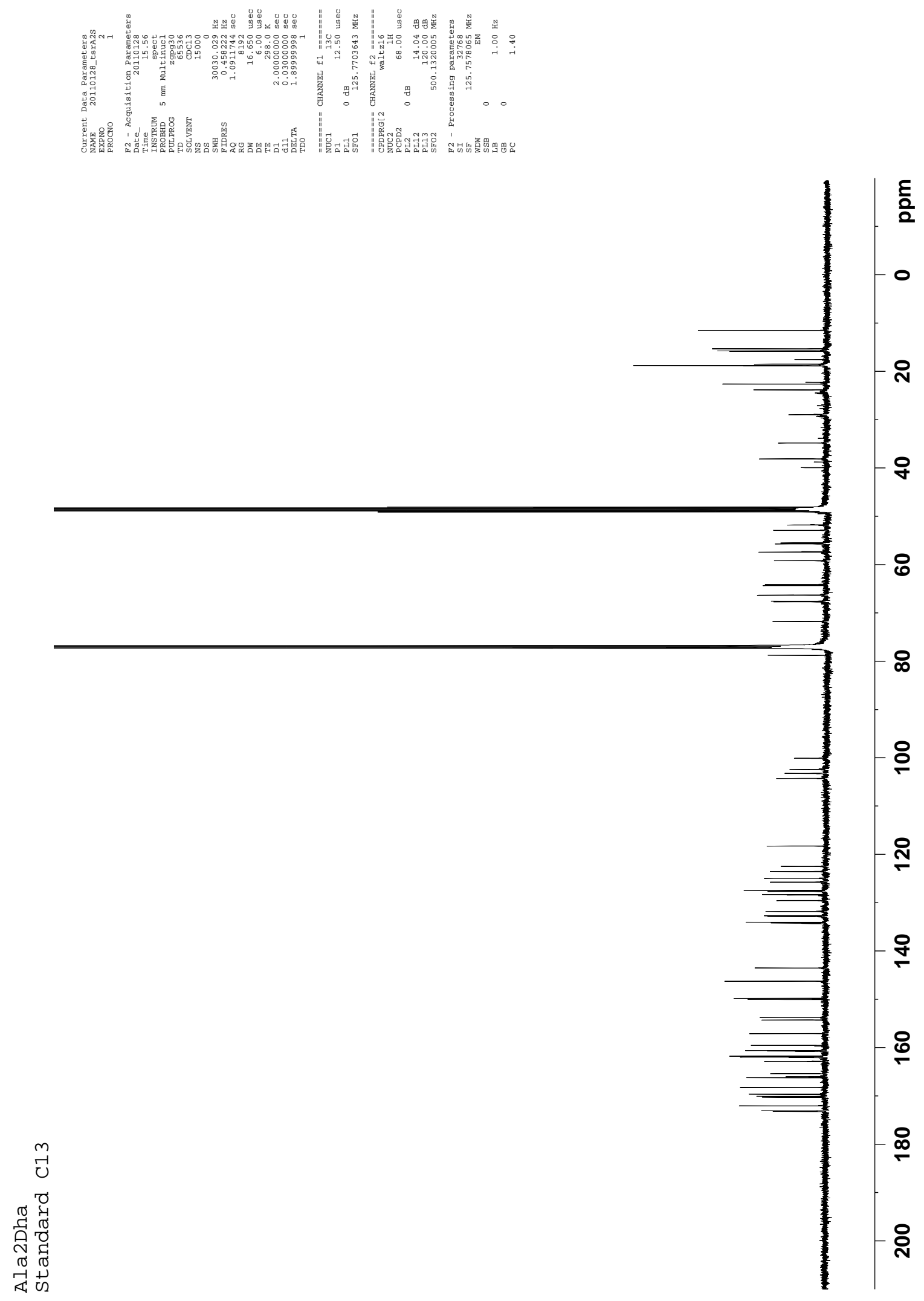

Figure S5. ${ }^{13} \mathrm{C}$ NMR spectrum of thiostrepton Ala2Dha $\left(125 \mathrm{MHz}, \mathrm{CDCl}_{3}-\mathrm{CD}_{3} \mathrm{OD} 4: 1,25{ }^{\circ} \mathrm{C}\right)$. 

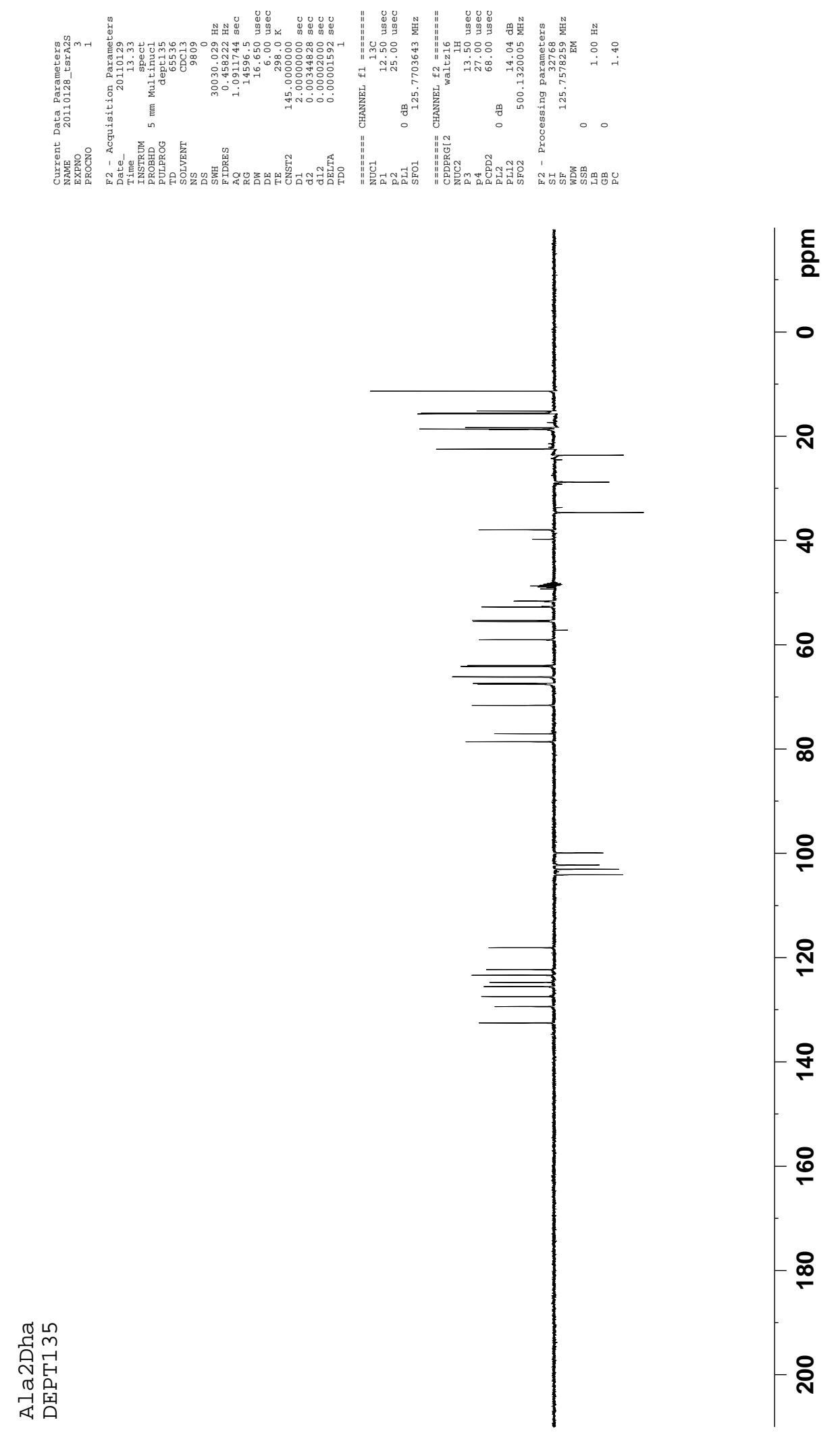

Figure S6. DEPT-135 NMR spectrum of thiostrepton Ala2Dha (125 MHz, $\mathrm{CDCl}_{3}-\mathrm{CD}_{3} \mathrm{OD}$ 4:1, $\left.25^{\circ} \mathrm{C}\right)$. 

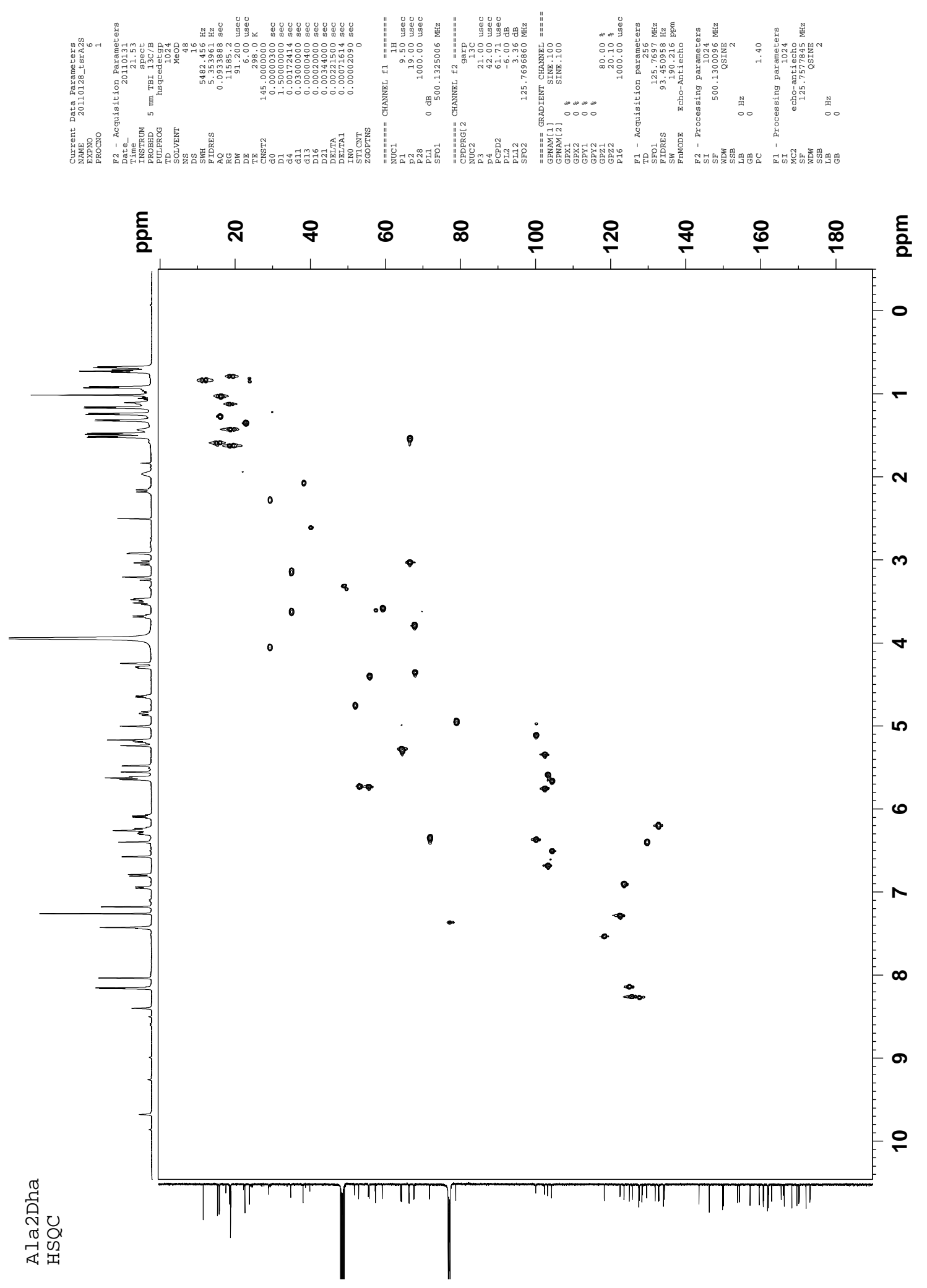

Figure S7. gHSQC spectrum of thiostrepton Ala2Dha (500 $\left.\mathrm{MHz}, \mathrm{CDCl}_{3}-\mathrm{CD}_{3} \mathrm{OD} 4: 1,25^{\circ} \mathrm{C}\right)$. 


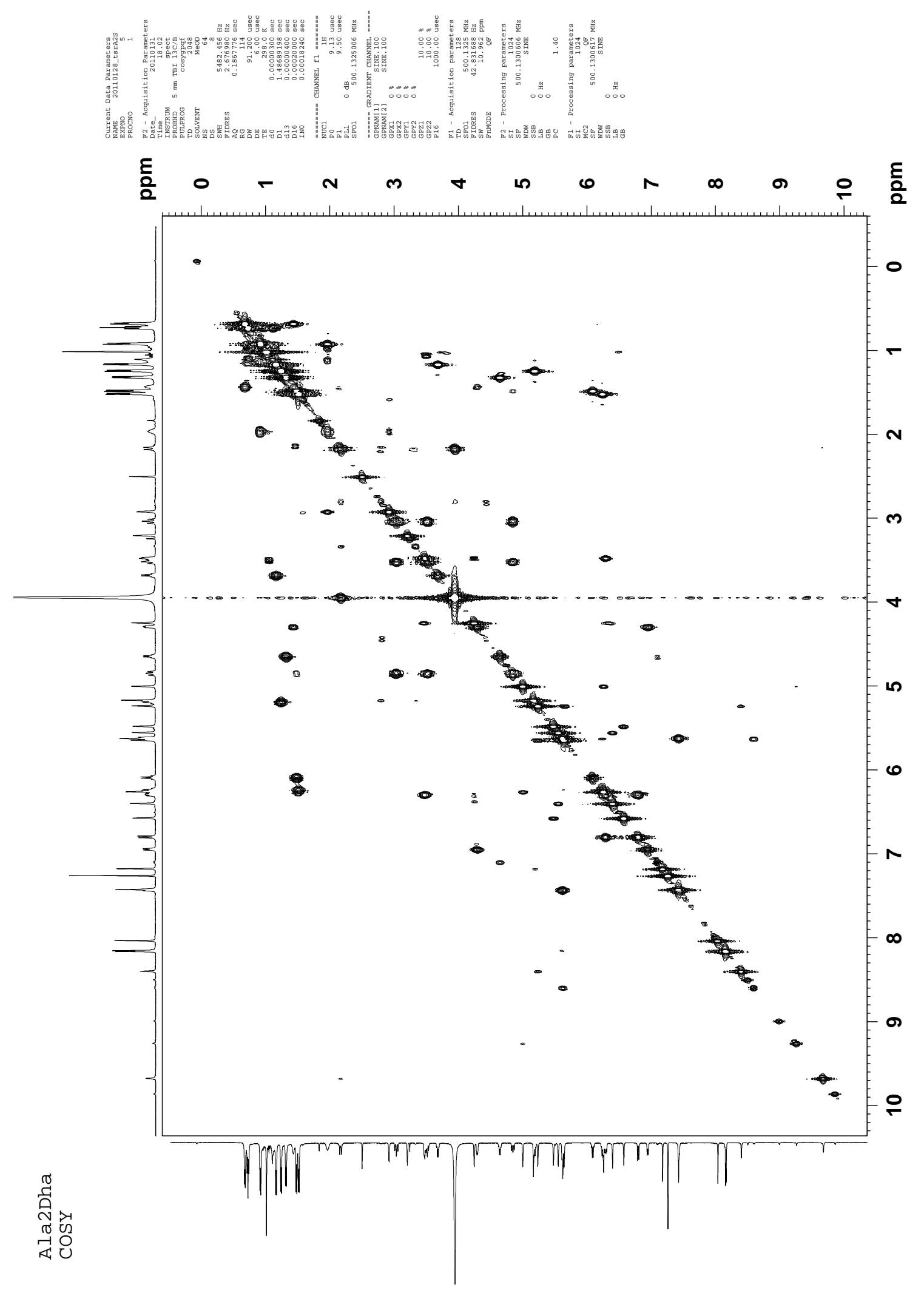

Figure S8. gCOSY spectrum of thiostrepton Ala2Dha (500 MHz, $\left.\mathrm{CDCl}_{3}-\mathrm{CD}_{3} \mathrm{OD} 4: 1,25^{\circ} \mathrm{C}\right)$. 


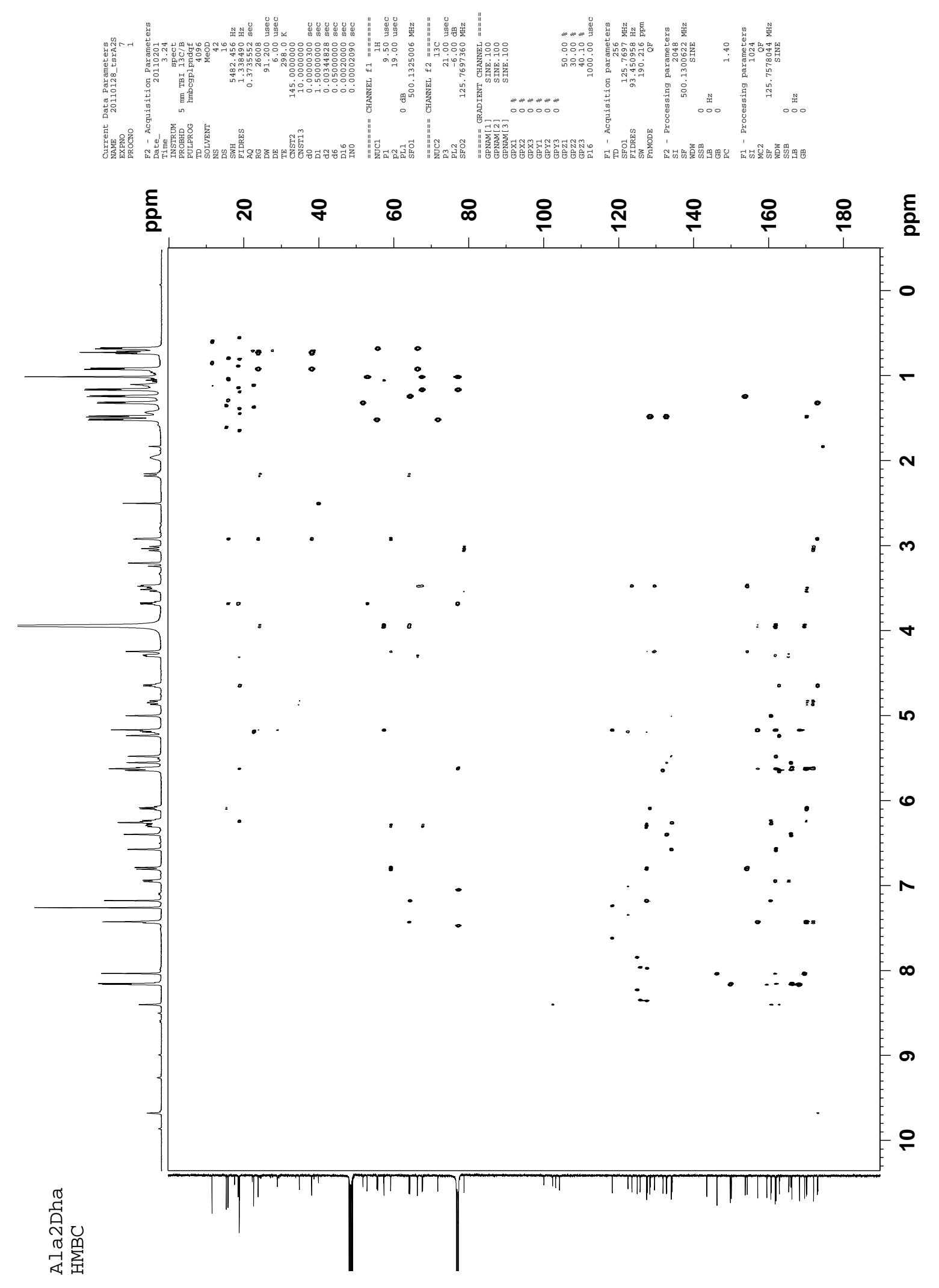

Figure S9. gHMBC spectrum of thiostrepton Ala2Dha (500 $\left.\mathrm{MHz}, \mathrm{CDCl}_{3}-\mathrm{CD}_{3} \mathrm{OD} 4: 1,25^{\circ} \mathrm{C}\right)$. 
Table S1. ${ }^{1} \mathrm{H}$ and ${ }^{13} \mathrm{C}$ NMR assignments of thiostrepton Ala2Dha

\begin{tabular}{|c|c|c|c|c|}
\hline Position & $\begin{array}{l}\delta_{\mathrm{C}}[\mathrm{ppm}] ; \\
\text { mult }\end{array}$ & $\begin{array}{l}\delta_{\mathrm{H}}[\mathrm{ppm}] ; \\
\text { (mult, } \mathrm{J} \text { in Hz) }\end{array}$ & HMBC $^{\text {a }}$ & $\operatorname{cosY}^{b}$ \\
\hline \multicolumn{5}{|l|}{ Ile1 } \\
\hline Ile1-1 & 173.0; $\mathrm{C} \mathrm{q}$ & & & \\
\hline Ile1-2 & $66.3 ; \mathrm{CH}$ & $2.93(\mathrm{~d}, 3.8)$ & Ile1-1; Ile1-3; Ile-4; Ile1-6; Q8 & Ile1-3 \\
\hline Ile1-3 & $38.1 ; \mathrm{CH}$ & $2.00-1.94(\mathrm{~m})$ & & 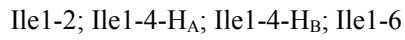 \\
\hline \multirow{2}{*}{ Ile1-4 } & $23.8 ; \mathrm{CH}_{2}$ & $\mathrm{H}_{\mathrm{A}}: 1.14-1.10(\mathrm{~m})$ & Ile1-5 & Ile1-3; Ile1-4-H ${ }_{B}$; Ile1-5 \\
\hline & & $\mathrm{H}_{\mathrm{B}}: 0.99-0.94(\mathrm{~m})$ & & Ile1-3, Ile1-4-H $\mathrm{H}_{\mathrm{A}}$; Ile1-5 \\
\hline Ile1-5 & $11.5 ; \mathrm{CH}_{3}$ & $0.73(\mathrm{t}, 7.1)$ & Ile1-3; Ile1-4 & Ile1-4- $\mathrm{H}_{\mathrm{A}} ;$ Ile1-4- $\mathrm{H}_{\mathrm{B}}$ \\
\hline Ile1-6 & $15.7 ; \mathrm{CH}_{3}$ & $0.92(\mathrm{~d}, 6.9)$ & Ile1-2; Ile1-3; Ile1-4 & Ile1-3 \\
\hline \multicolumn{5}{|l|}{ Dha2 } \\
\hline Dha2-1 & $160.7 ; \mathrm{C} \mathrm{q}$ & & & \\
\hline Dha2-2 & $134.2 ; \mathrm{C} \mathrm{q}$ & & & \\
\hline \multirow[t]{2}{*}{ Dha2-3 } & $100.1 ; \mathrm{CH}_{2}$ & $\mathrm{H}_{\mathrm{A}}: 6.27(\mathrm{br} \mathrm{s})$ & Dha2-1; Dha2-2 & Dha2-3- $\mathrm{H}_{\mathrm{B}}$ \\
\hline & & $\mathrm{H}_{\mathrm{B}}: 5.01(\mathrm{~d}, 1.4)$ & Dha2-1; Dha2-2 & Dha2-3- $\mathrm{H}_{\mathrm{A}}$ \\
\hline \multicolumn{5}{|l|}{ Dha3 } \\
\hline Dha3-1 & $162.8 ; \mathrm{C} \mathrm{q}$ & & & \\
\hline Dha3-2 & $131.8 ; \mathrm{C} \mathrm{q}$ & & & \\
\hline \multirow[t]{2}{*}{ Dha3-3 } & $102.4 ; \mathrm{CH}_{2}$ & $\mathrm{H}_{\mathrm{A}}: 5.65(\mathrm{~d}, 2.2)$ & Dha3-1; Dha3-2 & Dha3-3- $\mathrm{H}_{\mathrm{B}}$ \\
\hline & सר, & $\mathrm{H}_{\mathrm{B}}: 5.24(\mathrm{br} \mathrm{s})$ & Dha3-1 & Dha3-3-H $\mathrm{H}_{\mathrm{A}}$; Dha3-NH \\
\hline Dha3-NH ${ }^{\mathrm{d}}$ & & $8.40(\mathrm{~s})$ & Dha2-1; Dha3-1; Dha3-3 & Dha3-3- $\mathrm{H}_{\mathrm{B}}$ \\
\hline \multicolumn{5}{|l|}{ Ala4 } \\
\hline Ala4-1 & $173.1 ; \mathrm{C} \mathrm{q}$ & & & \\
\hline Ala4-2 & $51.8 ; \mathrm{CH}$ & $4.65(\mathrm{q}, 6.6)$ & Dha3-1; Ala4-1; Ala4-3 & Ala4-3; Ala4-NH \\
\hline Ala4-3 & $18.8 ; \mathrm{CH}_{3}$ & $1.32(\mathrm{~d}, 6.7)$ & Ala4-1; Ala4-2 & Ala4-2 \\
\hline Ala4-NH ${ }^{\mathrm{d}}$ & & $7.10(\mathrm{~d}, 8.2)$ & & Ala4-2 \\
\hline \multicolumn{5}{|l|}{ Pip } \\
\hline Pip-2 & 161.9; C q & & & \\
\hline \multirow[t]{2}{*}{ Pip-3 } & $24.5 ; \mathrm{CH}_{2}$ & $\mathrm{H}_{\mathrm{A}}: 3.36-3.32(\mathrm{~m})$ & & Pip-4- $\mathrm{H}_{\mathrm{B}}$ \\
\hline & & $\mathrm{H}_{\mathrm{B}}: 2.83-2.77(\mathrm{~m})$ & & Pip-4-H ${ }_{A} ;$ Pip-4- $H_{B}$ \\
\hline \multirow{2}{*}{ Pip-4 } & $28.9 ; \mathrm{CH}_{2}$ & $\mathrm{H}_{\mathrm{A}}: 3.95(\mathrm{~m})$ & Pip-2; Pip-3; Pip-5; Pip-6; Thz6-4; Thz13-2 & Pip-4- $\mathrm{H}_{\mathrm{B}}$ \\
\hline & & $\mathrm{H}_{\mathrm{B}}: 2.17(\mathrm{~m})$ & Pip-3; Pip-6 & Pip-3- $\mathrm{H}_{\mathrm{A}} ;$ Pip-4- $\mathrm{H}_{\mathrm{A}} ;$ Pip-3- $\mathrm{H}_{\mathrm{B}}$ \\
\hline Pip-5 & $57.3 ; \mathrm{C} \mathrm{q}$ & & & \\
\hline Pip-6 & $64.1 ; \mathrm{CH}$ & $5.17(\mathrm{~s})$ & Pip-2; Pip-4; Pip-5; Thz13-2; Thz13-3; Thz15-4 & Pip-3- $\mathrm{H}_{\mathrm{A}} ;$ Pip-3- $\mathrm{H}_{\mathrm{B}}$ \\
\hline \multicolumn{5}{|l|}{ Thz6 } \\
\hline Thz6-1 & $161.7 ; \mathrm{C} \mathrm{q}$ & & & \\
\hline Thz6-2 & 146.2; C q & & & \\
\hline Thz6-3 & $124.9 ; \mathrm{CH}$ & $8.04(\mathrm{~s})$ & Thz6-1; Thz6-2; Thz6-4 & \\
\hline Thz6-4 & $169.6 ; \mathrm{C} \mathrm{q}$ & & & \\
\hline \multicolumn{5}{|l|}{ Thr7 } \\
\hline Thr7-1 & $165.4 ; \mathrm{C} \mathrm{q}$ & & & \\
\hline Thr7-2 & $55.7 ; \mathrm{CH}$ & $4.30(\mathrm{dd}, 7.7,3.6)$ & Thz6-1; Thr7-1; Thr7-3; Thr7-4 & Thr7-3; Thr7-NH \\
\hline Thr7-3 & $66.3 ; \mathrm{CH}$ & $1.46-1.40(\mathrm{~m})$ & Thr7-4 & Thr7-2; Thr7-4 \\
\hline Thr7-4 & $18.8 ; \mathrm{CH}_{3}$ & $0.68(\mathrm{~d}, 6.3)$ & Thr7-2; Thr7-3 & Thr7-3 \\
\hline Thr7-NH ${ }^{\mathrm{d}}$ & & $6.95(d, 7.6)$ & Thz6-1; Thr7-1 & Thr7-2 \\
\hline \multicolumn{5}{|l|}{ Dhb8 } \\
\hline Dhb8-2 & $128.3 ; \mathrm{C} \mathrm{q}$ & & & \\
\hline Dhb8-3 & $132.7 ; \mathrm{CH}$ & $6.10(\mathrm{q}, 7.0)$ & Dhb8-2; Dhb8-4; Tzn9-4 & Dhb8-4 \\
\hline Dhb8-4 & $15.3 ; \mathrm{CH}_{3}$ & $1.48(\mathrm{~d}, 7.3)$ & Dhb8-2; Dhb8-3; Tzn9-4 & Dhb8-3 \\
\hline \multicolumn{5}{|l|}{ Tzn9 } \\
\hline Tzn9-1 & 172.0; C q & & & \\
\hline Tzn9-2 & $78.8 ; \mathrm{CH}$ & $4.85(\mathrm{dd}, 12.8,9.0)$ & Tzn9-1; Tzn9-3; Tzn9-4 & Tzn9-3- $\mathrm{H}_{\mathrm{A}} ; \mathrm{Tzn} 9-3-\mathrm{H}_{\mathrm{B}}$ \\
\hline \multirow[t]{2}{*}{ Tzn9-3 } & $34.8 ; \mathrm{CH}_{2}$ & $\mathrm{H}_{\mathrm{A}}: 3.52(\mathrm{dd}, 11.2,9.0)$ & Tzn9-2; Tzn9-4 & Tzn9-2; Tzn9-3- $\mathrm{H}_{\mathrm{B}}$ \\
\hline & & $\mathrm{H}_{\mathrm{B}}: 3.04(\mathrm{dd}, 12.8,11.5)$ & Tzn9-1; Tzn9-2 & Tzn9-2; Tzn9-3- $\mathrm{H}_{\mathrm{A}}$ \\
\hline Tzn9-4 & $170.2 ; \mathrm{C} \mathrm{q}$ & & & \\
\hline \multicolumn{5}{|l|}{ Ile10 } \\
\hline Ile10-2 & $52.9 ; \mathrm{CH}$ & $5.62(\mathrm{~d}, 5.1)$ & Tzn9-1; Ile10-3; Thz11-4 & Ile10-NH \\
\hline Ile10-3 & $77.1^{\mathrm{c}} ; \mathrm{C} \mathrm{q}$ & & & \\
\hline Ile $10-4$ & $67.6 ; \mathrm{CH}$ & $3.68(\mathrm{q}, 6.4)$ & Ile10-2; Ile10-3; Ile10-5; Ile10-6 & Ile 10-5 \\
\hline Ile $10-5$ & $15.8 ; \mathrm{CH}_{3}$ & $1.17(\mathrm{~d}, 6.3)$ & Ile10-3; Ile10-4 & Ile10-4 \\
\hline Ile10-6 & $18.5 ; \mathrm{CH}_{3}$ & $1.02(\mathrm{~s})$ & Ile10-2; Ile10-3; Ile10-4 & \\
\hline Ile10-NH ${ }^{\mathrm{d}}$ & & $7.43(\mathrm{~d}, 9.8)$ & Tzn9-1 & Ile 10-2 \\
\hline
\end{tabular}




\begin{tabular}{|c|c|c|c|c|}
\hline Position & $\begin{array}{l}\delta_{\mathrm{C}}[\mathrm{ppm}] \\
\text { mult }\end{array}$ & $\begin{array}{l}\delta_{\mathrm{H}}[\mathrm{ppm}] ; \\
\text { (mult, } \mathrm{J} \text { in } \mathrm{Hz})\end{array}$ & НМBC $^{\mathbf{a}}$ & $\operatorname{cosY}^{b}$ \\
\hline \multicolumn{5}{|l|}{ Thz11 } \\
\hline $\begin{array}{l}\text { Thz11-1 } \\
\text { Thz11-2 } \\
\text { Thz11-3 } \\
\text { Thz11-4 }\end{array}$ & $\begin{array}{l}162.0 ; \mathrm{C} \mathrm{q} \\
150.0 ; \mathrm{C} \mathrm{q} \\
125.7 ; \mathrm{CH} \\
166.2 ; \mathrm{C} \mathrm{q}\end{array}$ & $8.16(\mathrm{~s})$ & Thz11-1; Thz11-2; Thz11-4 & \\
\hline $\begin{array}{l}\text { Thr12 } \\
\text { Thr12-2 } \\
\text { Thr12-3 } \\
\text { Thr12-4 } \\
\text { Thr12-NH }\end{array}$ & $\begin{array}{l}55.5 ; \mathrm{CH} \\
71.8 ; \mathrm{CH} \\
18.8 ; \mathrm{CH}_{3}\end{array}$ & $\begin{array}{l}5.64(\mathrm{~d}, 4.1) \\
6.25(\mathrm{q}, 6.7) \\
1.52(\mathrm{~d}, 6.3) \\
8.60(\mathrm{~d}, 8.9)\end{array}$ & $\begin{array}{l}\text { Thz11-1; Thr12-4; Thz13-2; Thz13-4 } \\
\text { Thr12-4; Thz13-4; Q-1 } \\
\text { Thr12-2; Thr12-3 }\end{array}$ & $\begin{array}{l}\text { Thr12-3, Thr12-NH } \\
\text { Thr12-2, Thr12-4 } \\
\text { Thr12-3 } \\
\text { Thr12-2 }\end{array}$ \\
\hline $\begin{array}{l}\text { Thz13 } \\
\text { Thz13-2 } \\
\text { Thz13-3 } \\
\text { Thz13-4 }\end{array}$ & $\begin{array}{l}157.1 ; \mathrm{C} \mathrm{q} \\
118.3 ; \mathrm{CH} \\
170.0 ; \mathrm{C} \mathrm{q}\end{array}$ & $7.43(\mathrm{~s})$ & Pip-6; Thz13-2; Thz13-4 & \\
\hline $\begin{array}{l}\text { Thz15 } \\
\text { Thz15-1 } \\
\text { Thz15-2 } \\
\text { Thz15-3 } \\
\text { Thz15-4 }\end{array}$ & $\begin{array}{l}159.5 ; \mathrm{C} \mathrm{q} \\
149.8 ; \mathrm{C} \mathrm{q} \\
127.6 ; \mathrm{CH} \\
168.2 ; \mathrm{C} \mathrm{q}\end{array}$ & $8.17(\mathrm{~s})$ & Thz15-1; Thz15-2; Thz15-4 & \\
\hline $\begin{array}{l}\text { Dha16 } \\
\text { Dha16-1 } \\
\text { Dha16-2 } \\
\text { Dha16-3 }\end{array}$ & $\begin{array}{l}162.0 ; \mathrm{C} \mathrm{q} \\
134.0 ; \mathrm{C} \mathrm{q} \\
103.2 ; \mathrm{CH}_{2}\end{array}$ & $\begin{array}{l}\mathrm{H}_{\mathrm{A}}: 6.58(\mathrm{~d}, 2.2) \\
\mathrm{H}_{\mathrm{B}}: 5.48(\mathrm{~d}, 2.2)\end{array}$ & $\begin{array}{l}\text { Dha16-1; Dha16-2 } \\
\text { Dha16-1; Dha16-2 }\end{array}$ & $\begin{array}{l}\text { Dha16-3- } \mathrm{H}_{\mathrm{B}} \\
\text { Dha16-3- } \mathrm{H}_{\mathrm{A}}\end{array}$ \\
\hline \multicolumn{5}{|l|}{ Dha17 } \\
\hline $\begin{array}{l}\text { Dha17-1 } \\
\text { Dha17-2 } \\
\text { Dha17-3 }\end{array}$ & $\begin{array}{l}166.0 ; \mathrm{C} \mathrm{q} \\
132.8 ; \mathrm{C} \mathrm{q} \\
104.3 ; \mathrm{CH}_{2}\end{array}$ & $\begin{array}{l}\mathrm{H}_{\mathrm{A}}: 6.41(\mathrm{~d}, 1.2) \\
\mathrm{H}_{\mathrm{B}}: 5.56(\mathrm{~d}, 1.6)\end{array}$ & $\begin{array}{l}\text { Dha17-1; Dha17-2 } \\
\text { Dha17-1; Dha17-2 }\end{array}$ & $\begin{array}{l}\text { Dha17-3- } \mathrm{H}_{\mathrm{B}} \\
\text { Dha17-3- } \mathrm{H}_{\mathrm{A}}\end{array}$ \\
\hline \multicolumn{5}{|c|}{ 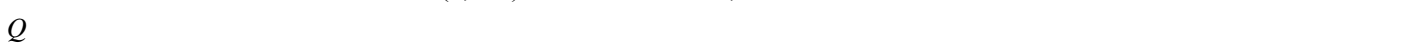 } \\
\hline $\begin{array}{l}\text { Q-1 } \\
\text { Q-2 } \\
\text { Q-3 } \\
\text { Q-4 } \\
\text { Q-5 }\end{array}$ & $\begin{array}{l}160.6 ; \mathrm{C} \mathrm{q} \\
143.5 ; \mathrm{C} \mathrm{q} \\
122.5 ; \mathrm{CH} \\
153.8 ; \mathrm{C} \mathrm{q} \\
127.4 ; \mathrm{C} \mathrm{q}\end{array}$ & $7.18(\mathrm{~s})$ & Q-1; Q-5; Q-11 & \\
\hline Q-6 & 123.5; CH & $6.80(\mathrm{~d}, 10.1)$ & Q-5; Q-8; Q-10 & Q-7 \\
\hline Q-7 & 129.5; $\mathrm{CH}$ & $6.30(\mathrm{dd}, 9.8,5.7)$ & Q-5; Q-8; Q-9 & Q-6; Q-8; Q-9 \\
\hline Q-8 & $59.2 ; \mathrm{CH}$ & $3.48(\mathrm{dd}, 5.9,1.8)$ & Q-6; Q-7; Q-9; Q-10; Ile1-2 & Q-7; Q-9 \\
\hline $\begin{array}{l}\text { Q-9 } \\
\text { Q-10 }\end{array}$ & $\begin{array}{l}\text { 67.7; } \mathrm{CH} \\
154.3 ; \mathrm{C} \mathrm{q}\end{array}$ & $4.25(\mathrm{~s})$ & Q-5; Q-7; Q-8; Q-10 & Q-7; Q-8 \\
\hline Q-11 & 64.3; $\mathrm{CH}$ & $5.19(\mathrm{q}, 6.6)$ & Q-3; Q-5; Q-12 & Q-12 \\
\hline Q-12 & $22.6 ; \mathrm{CH}_{3}$ & $1.25(\mathrm{~d}, 6.6)$ & Q-4; Q-11 & Q-11 \\
\hline
\end{tabular}

${ }^{a} \mathrm{HMBC}$ correlations are from the proton to the indicated carbon.

${ }^{\mathrm{b}} \mathrm{COSY}$ correlations are from the proton to the proton attached to the indicated position.

${ }^{\mathrm{c}}$ The $\delta$ of this resonance was determined by HMBC due to overlap with the $\mathrm{CDCl}_{3}$ resonance.

${ }^{\mathrm{d}}$ Only those amide resonances demonstrating HMBC or COSY correlations to neighboring carbons or protons, respectively, were assigned. 
Figure S10. MS analysis of thiostrepton Ala2Dhb isolated from S. laurentii NDS1/int-A2T.

(A) HPLC-MS analysis. (1) Chromatogram extracted for $\mathrm{m} / \mathrm{z} 838.8$, the calculated $[\mathrm{M}+2 \mathrm{H}]^{2+}$ ion of thiostrepton Ala2Dhb. (2) Total ion chromatogram.

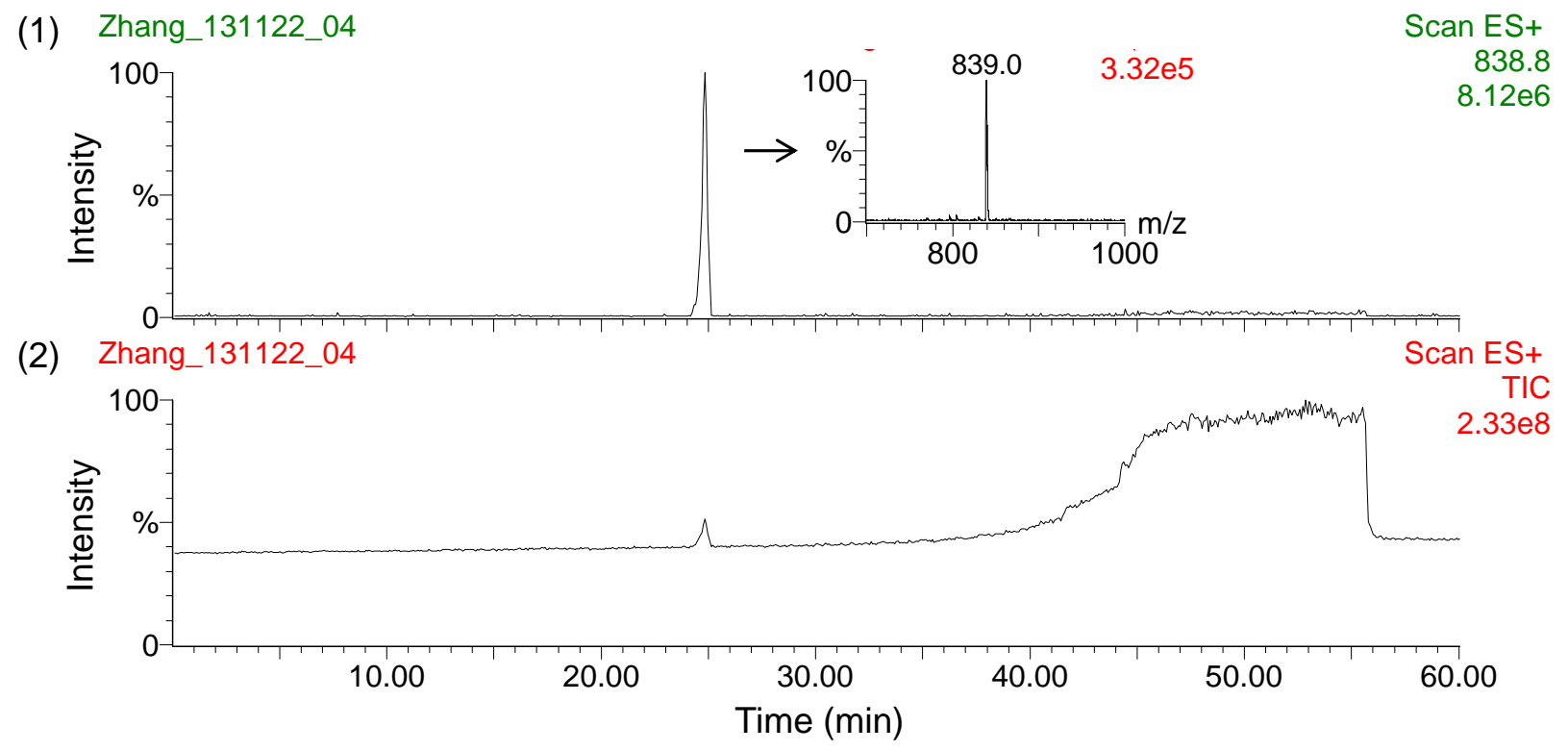

(B) MALDI MS spectrum of thiostrepton Ala2Dhb.

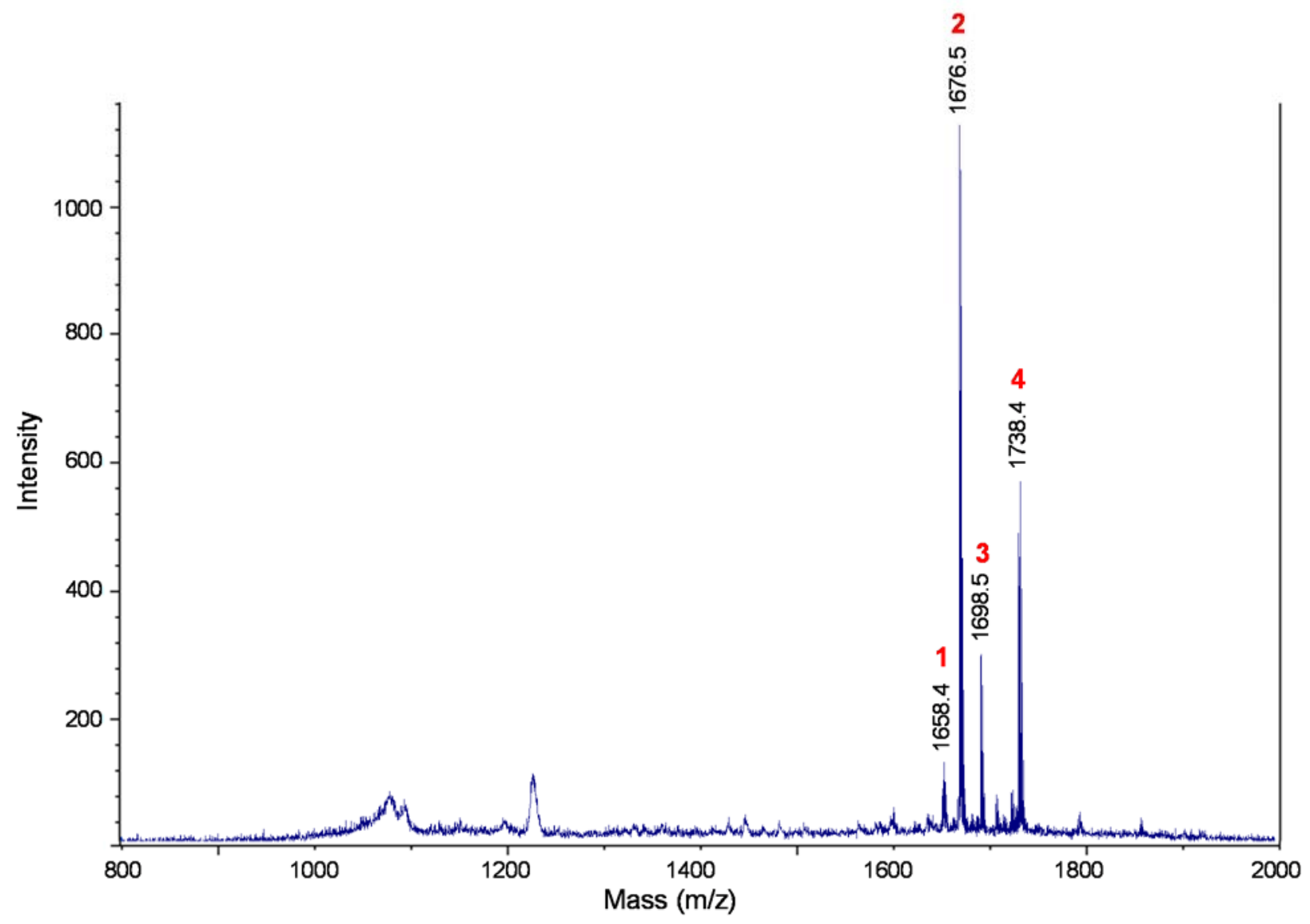


(C) MALDI MS/MS of parent ion $\mathrm{m} / \mathrm{z}$ 1676.5.

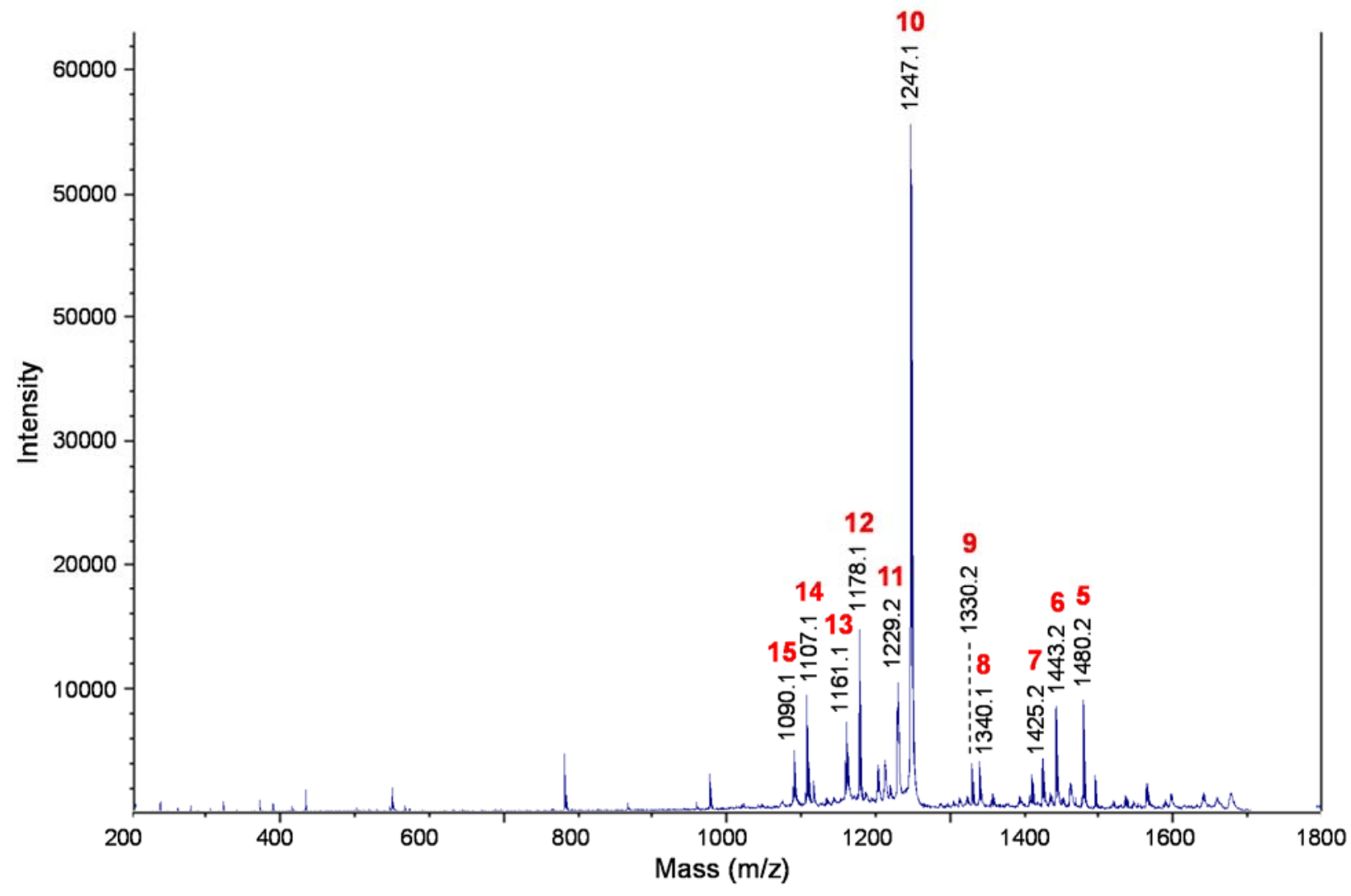

(D) Table and structure showing key ions and fragments in the MALDI MS and MS/MS of thiostrepton Ala2Dhb.

\begin{tabular}{|c|c|c|}
\hline Fragment & Expected & Observed \\
\hline 1. $\mathrm{M}-\mathrm{H}_{2} \mathrm{O}+\mathrm{H}^{+}$ & 1658.5 & 1658.4 \\
\hline 2. $\mathrm{M}+\mathrm{H}^{+}$(Parent ion) & 1676.5 & 1676.5 \\
\hline 3. $\mathrm{M}+\mathrm{Na}^{+}$ & 1698.5 & 1698.5 \\
\hline 4. $\mathrm{M}+\mathrm{Cu}^{+}$ & 1738.4 & 1738.4 \\
\hline 5. M-lle1-Dhb2+H+ & 1480.4 & 1480.2 \\
\hline 6. $\mathrm{M}-\mathrm{QA}+\mathrm{H}^{+}$ & 1443.4 & 1443.2 \\
\hline 7. $\mathrm{M}-\mathrm{QA}-\mathrm{H}_{2} \mathrm{O}+\mathrm{H}^{+}$ & 1425.4 & 1425.2 \\
\hline 8. M-Ile1-Dhb2-Dha3-Ala4+H+ ${ }^{+}$ & 1340.3 & 1340.1 \\
\hline 9. $\mathrm{M}-\mathrm{QA}-\mathrm{Ile} 1+\mathrm{H}^{+}$ & 1330.3 & 1330.2 \\
\hline 10. M-QA-Ile1-Dhb2+H+ & 1247.3 & 1247.1 \\
\hline 11. M-QA-Ile1-Dhb2- $\mathrm{H}_{2} \mathrm{O}+\mathrm{H}^{+}$ & 1229.3 & 1229.2 \\
\hline 12. M-QA-Ile1-Dhb2-Dha3+H+ & 1178.3 & 1178.1 \\
\hline 13. M-QA-Ile1-Dhb2-Dha3-OH+H+ & 1161.3 & 1161.1 \\
\hline 14. M-QA-Ile1-Dhb2-Dha3-Ala4+H+ & 1107.3 & 1107.1 \\
\hline 15. M-QA-Ile1-Dhb2-Dha3-Ala4- $\mathrm{OH}+\mathrm{H}^{+}$ & 1090.2 & 1090.1 \\
\hline
\end{tabular}

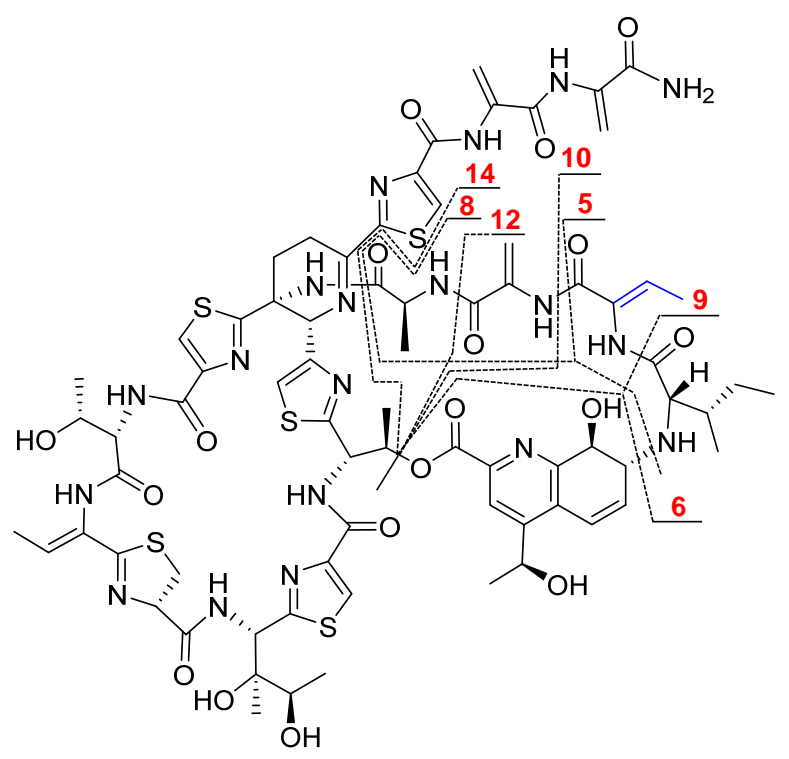


Figure S11. Structure and numbering system used for thiostrepton Ala2Dhb.

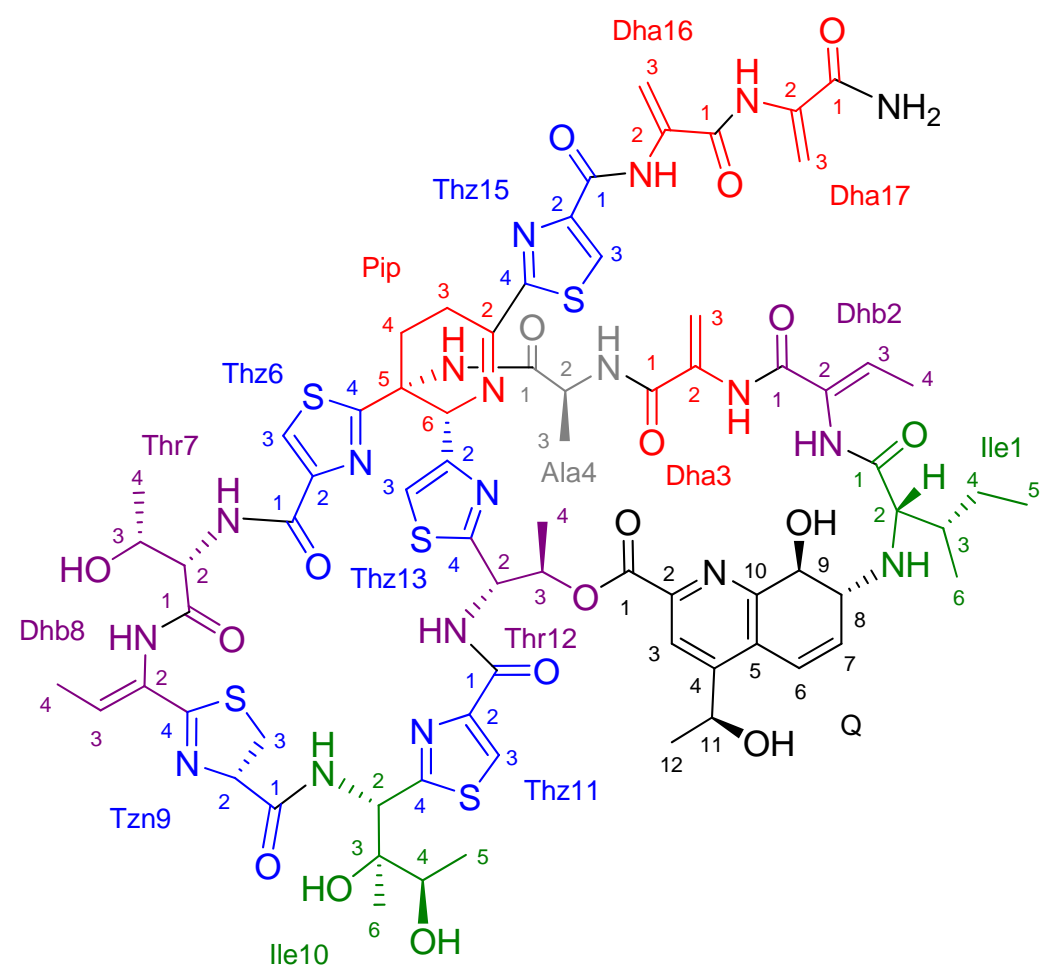




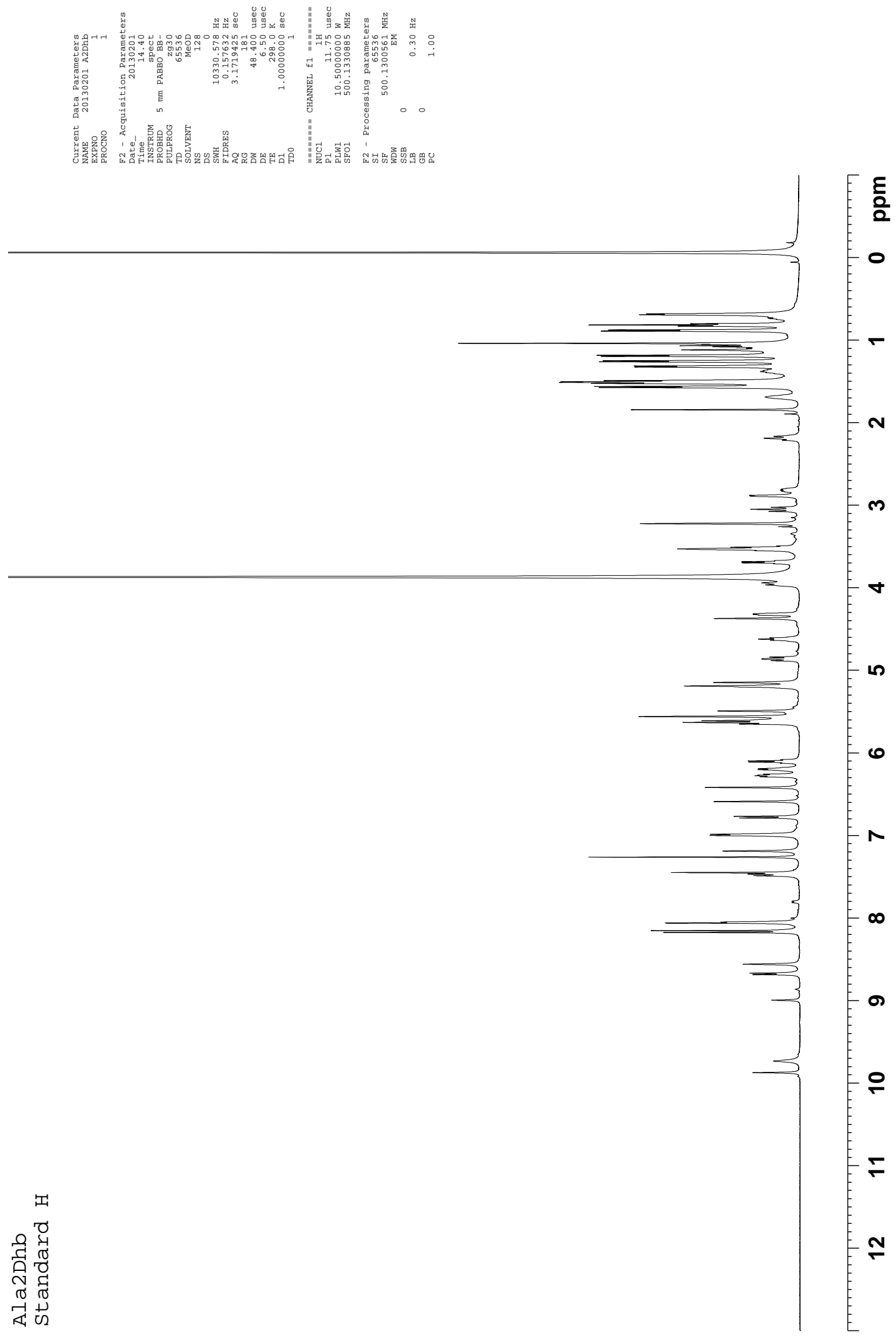

Figure S12. ${ }^{1} \mathrm{H}$ NMR spectrum of thiostrepton Ala2Dhb $\left(500 \mathrm{MHz}, \mathrm{CDCl}_{3}-\mathrm{CD}_{3} \mathrm{OD} 4: 1,25{ }^{\circ} \mathrm{C}\right)$. 

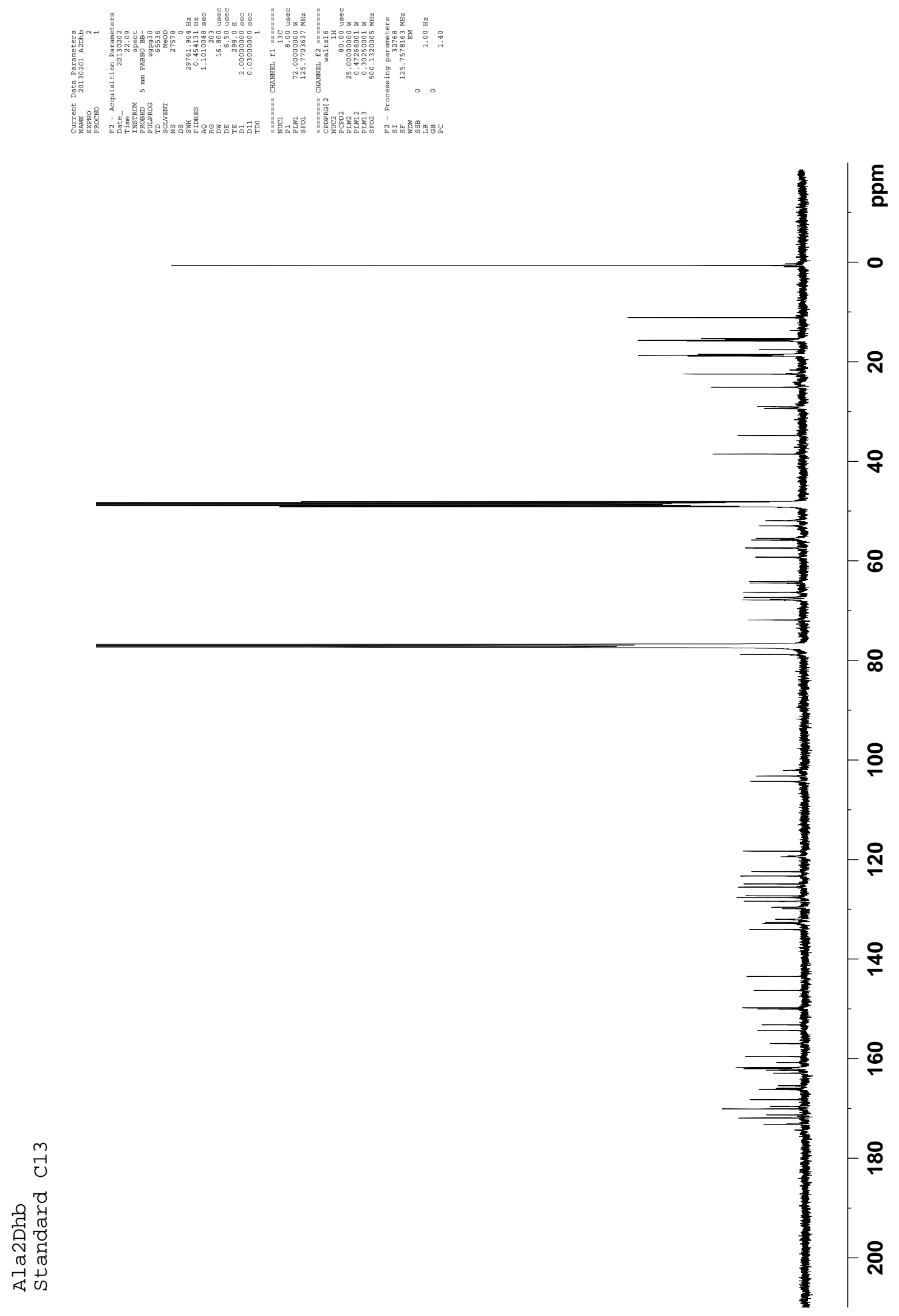

Figure S13. ${ }^{13} \mathrm{C}$ NMR spectrum of thiostrepton Ala2Dhb $\left(125 \mathrm{MHz}, \mathrm{CDCl}_{3}-\mathrm{CD}_{3} \mathrm{OD} 4: 1,25{ }^{\circ} \mathrm{C}\right)$. 

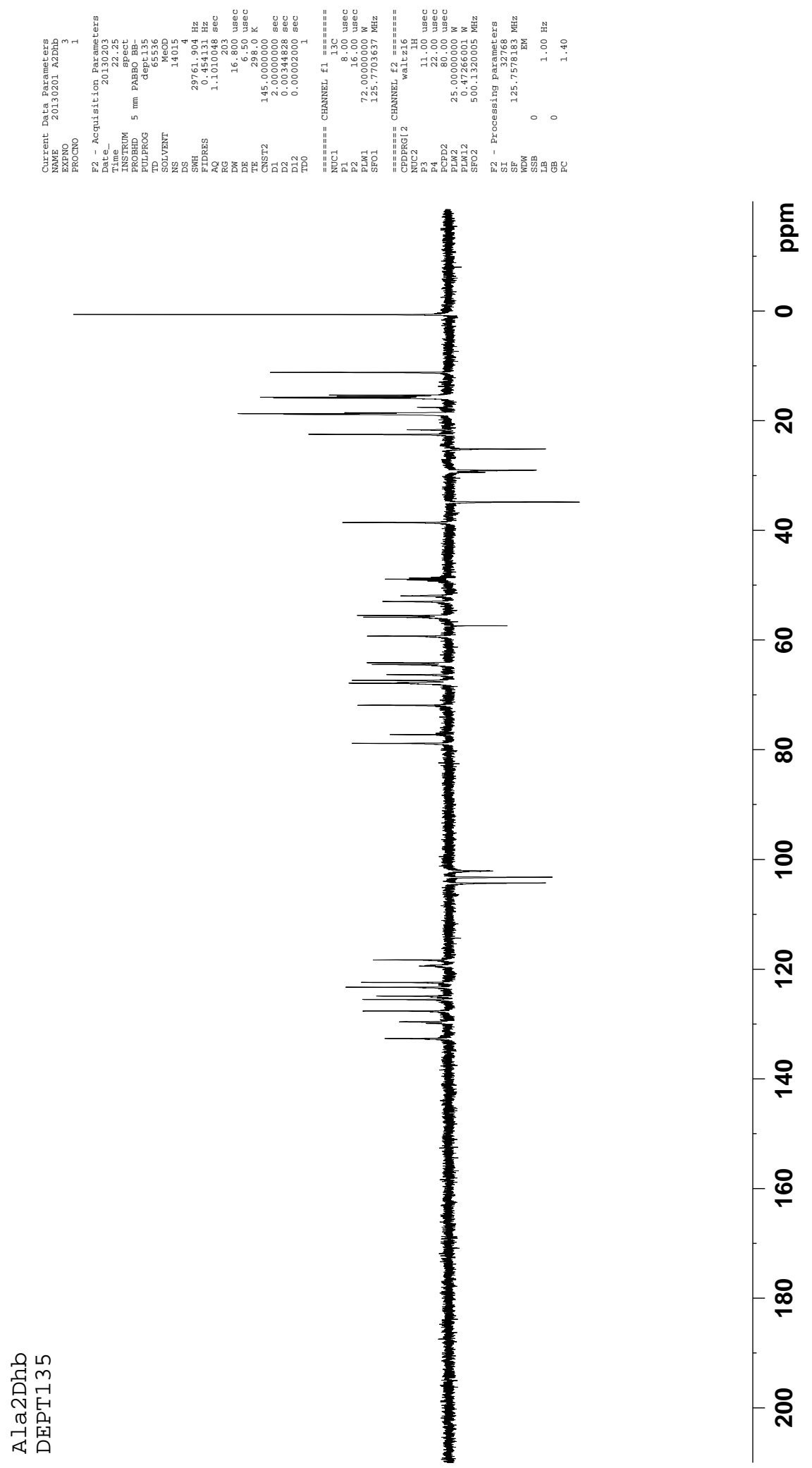

Figure S14. DEPT-135 NMR spectrum of thiostrepton Ala2Dhb (125 MHz, $\mathrm{CDCl}_{3}-\mathrm{CD}_{3} \mathrm{OD} 4: 1$, $25^{\circ} \mathrm{C}$ ). 


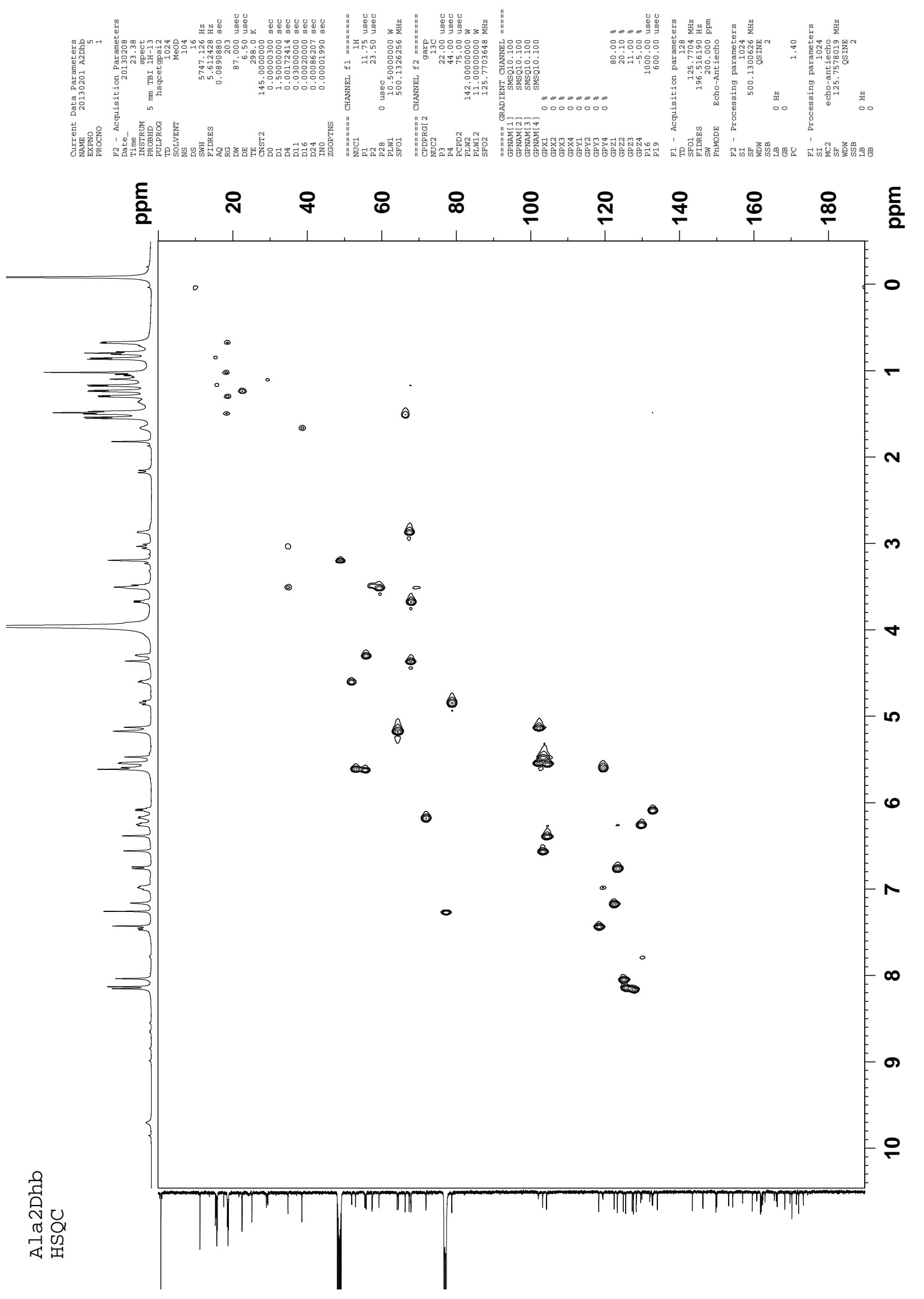

Figure S15. gHSQC spectrum of thiostrepton Ala2Dhb (500 MHz, $\left.\mathrm{CDCl}_{3}-\mathrm{CD}_{3} \mathrm{OD} 4: 1,25{ }^{\circ} \mathrm{C}\right)$. 


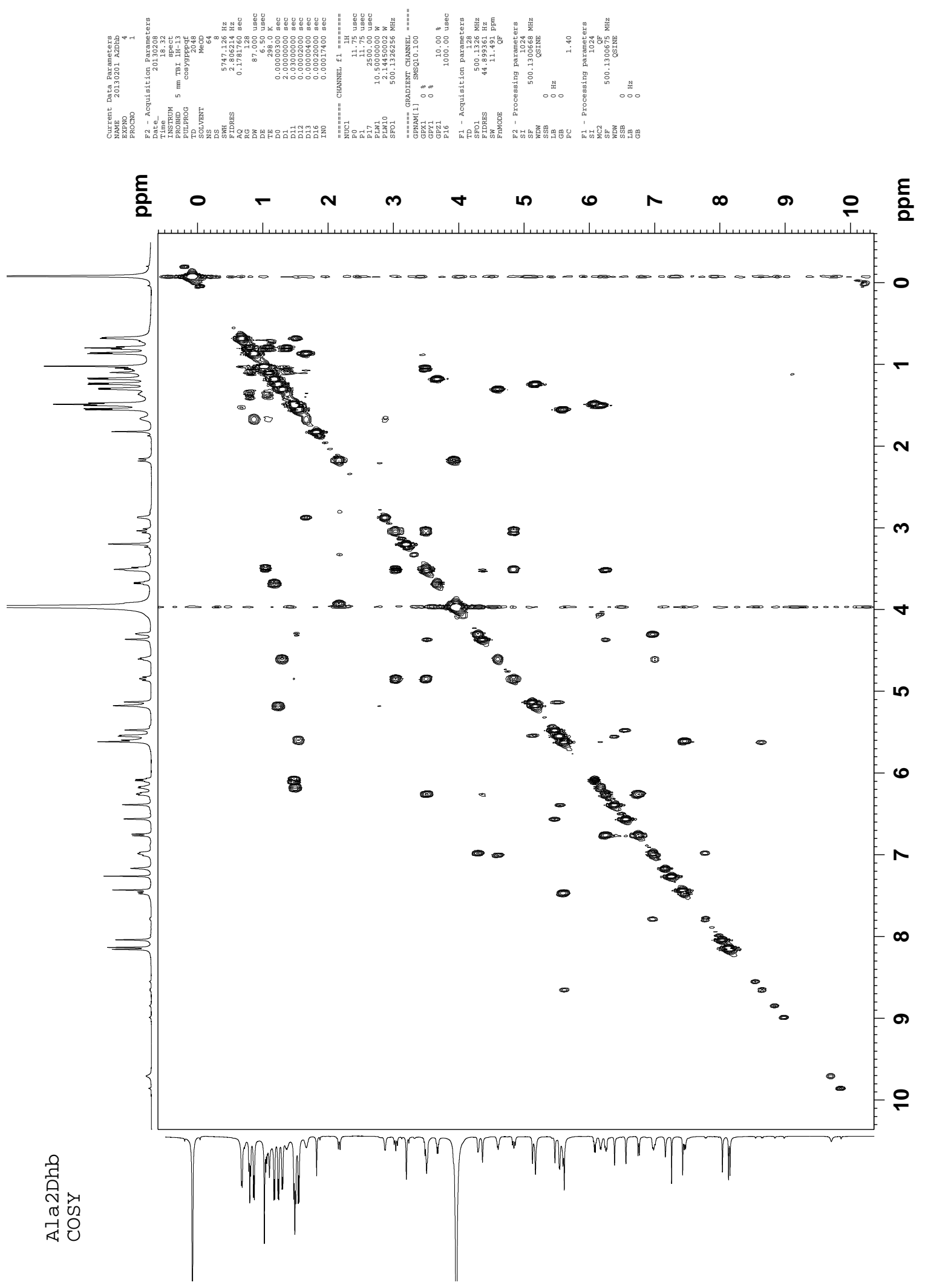

Figure S16. gCOSY spectrum of thiostrepton Ala2Dhb $\left(500 \mathrm{MHz}, \mathrm{CDCl}_{3}-\mathrm{CD}_{3} \mathrm{OD} 4: 1,25^{\circ} \mathrm{C}\right)$. 


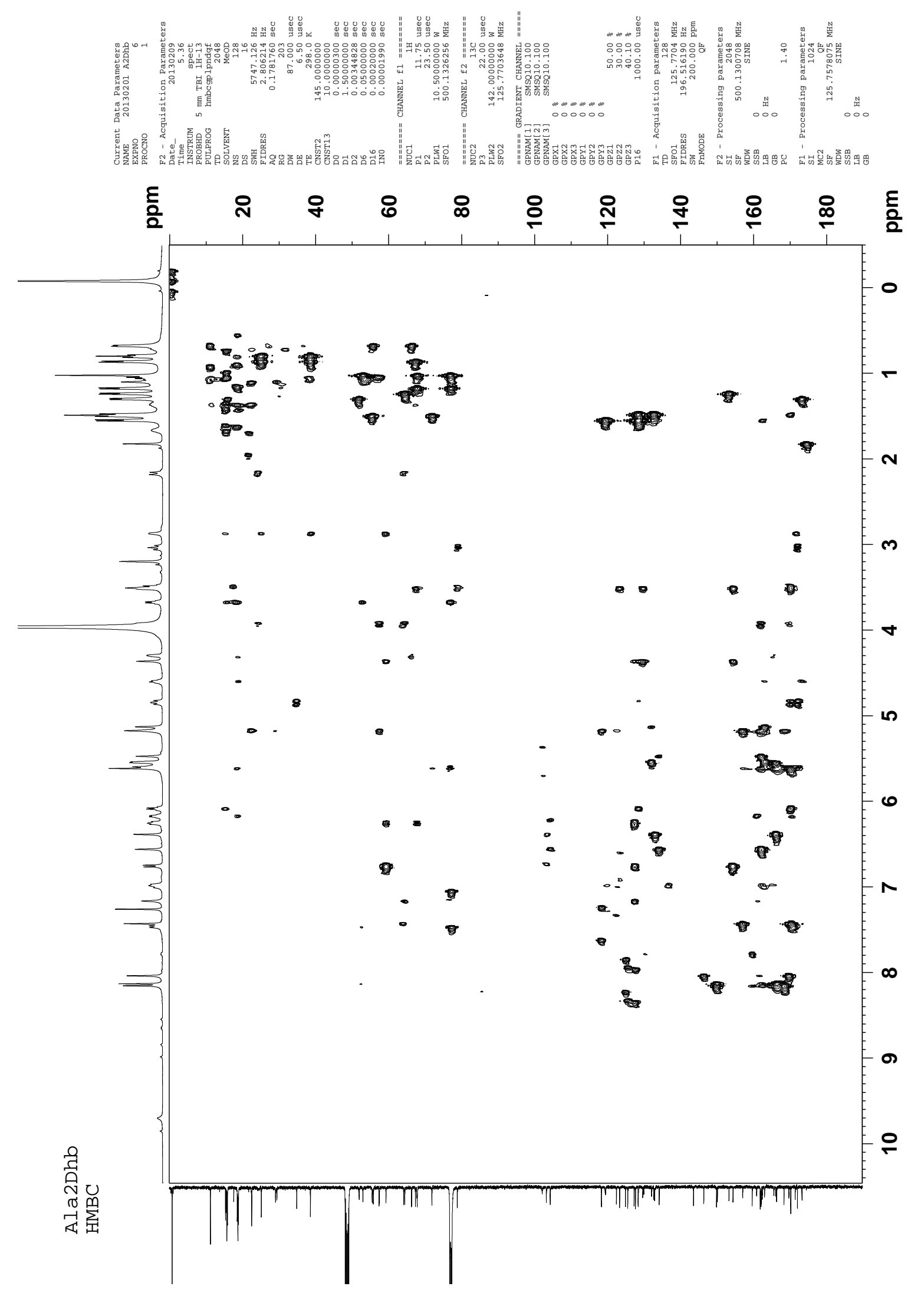

Figure S17. gHMBC spectrum of thiostrepton Ala2Dhb (500 MHz, $\left.\mathrm{CDCl}_{3}-\mathrm{CD}_{3} \mathrm{OD} 4: 1,25{ }^{\circ} \mathrm{C}\right)$. 
Table S2. ${ }^{1} \mathrm{H}$ and ${ }^{13} \mathrm{C}$ NMR assignments of thiostrepton Ala2Dhb

\begin{tabular}{|c|c|c|c|c|}
\hline Position & $\begin{array}{l}\delta_{\mathrm{C}}[\mathbf{p p m}] ; \\
\text { mult }\end{array}$ & $\begin{array}{l}\delta_{\mathrm{H}}[\mathrm{ppm}] \\
(\mathrm{mult}, \mathrm{J} \text { in } \mathrm{Hz})\end{array}$ & $\mathbf{H M B C}^{\mathbf{a}}$ & $\operatorname{COSY}^{\mathbf{b}}$ \\
\hline \multicolumn{5}{|l|}{ Ile1 } \\
\hline Ile1-1 & 171.3; C q & & & \\
\hline Ile1-2 & $67.3 ; \mathrm{CH}$ & $2.87(\mathrm{~d}, 4.1)$ & Ile1-1; Ile1-3; Ile1-4; Ile1-6; Q-8 & Ile1-3 \\
\hline Ile1-3 & $38.5 ; \mathrm{CH}$ & $1.70-1.63(\mathrm{~m})$ & Ile1-6 & 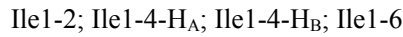 \\
\hline \multirow[t]{2}{*}{ Ile $1-4$} & $25.1 ; \mathrm{CH}_{2}$ & $\mathrm{H}_{\mathrm{A}}: 1.41-1.33(\mathrm{~m})$ & Ile1-2; Ile1-5; Ile1-6 & Ile1-3; Ile1-4-H \\
\hline & & $\mathrm{H}_{\mathrm{B}}: 1.12-1.07(\mathrm{~m})$ & Ile1-2; Ile1-3; Ile1-5; Ile1-6 & Ile1-3; Ile1-4-H $\mathrm{H}_{\mathrm{A}}$; Ile1-5 \\
\hline Ile1-5 & $11.2 ; \mathrm{CH}_{3}$ & $0.80(\mathrm{t}, 6.9)$ & Ile1-3; Ile1-4 & Ile1-4- $\mathrm{H}_{\mathrm{A}} ;$ Ile1-4- $\mathrm{H}_{\mathrm{B}}$ \\
\hline Ile1-6 & $15.3 ; \mathrm{CH}_{3}$ & $0.86(\mathrm{~d}, 6.7)$ & Ile1-2; Ile1-3; Ile1-4 & Ile1-3 \\
\hline \multicolumn{5}{|l|}{ Dhb2 } \\
\hline Dhb2-1 & $162.1 ; \mathrm{Cq}$ & & & \\
\hline Dhb2-2 & $128.4 ; \mathrm{C} \mathrm{q}$ & & & \\
\hline Dhb2-3 & $119.4 ; \mathrm{CH}$ & $5.64-5.60(\mathrm{~m})$ & Dhb2-1; Dhb2-4 & Dhb2-4 \\
\hline Dhb2-4 & $18.5 ; \mathrm{CH}_{3}$ & $1.55(\mathrm{~d}, 7.0)$ & Dhb2-1; Dhb2-2; Dhb2-3 & Dhb2-3 \\
\hline Dhb2-NH ${ }^{d}$ & & $7.02-6.94(\mathrm{~m})$ & Dha2-1; Dhb2-3 & \\
\hline \multicolumn{5}{|l|}{ Dha3 } \\
\hline Dha3-1 & $163.0 ; \mathrm{C} \mathrm{q}$ & & & \\
\hline Dha3-2 & $132.0 ; \mathrm{C} \mathrm{q}$ & & & \\
\hline \multirow[t]{2}{*}{ Dha3-3 } & $102.1 ; \mathrm{CH}_{2}$ & $\mathrm{H}_{\mathrm{A}}: 5.54(\mathrm{~s})$ & Dha3-1; Dha3-2 & Dha3-3-H \\
\hline & & $\mathrm{H}_{\mathrm{B}}: 5.13(\mathrm{~s})$ & Dha3-1; Dha3-2 & Dha3-3- $\mathrm{H}_{\mathrm{A}}$ \\
\hline \multicolumn{5}{|l|}{ Ala4 } \\
\hline Ala4-1 & $173.2 ; \mathrm{C} \mathrm{q}$ & & & \\
\hline Ala4-2 & $51.9 ; \mathrm{CH}$ & $4.60(\mathrm{q}, 6.0)$ & Dha3-1; Ala4-1; Ala4-3 & Ala4-3; Ala4-NH \\
\hline Ala4-3 & $18.8 ; \mathrm{CH}_{3}$ & $1.30(\mathrm{~d}, 6.2)$ & Ala4-1; Ala4-2 & Ala4-2 \\
\hline Ala4-NH ${ }^{d}$ & & $7.02-6.94(\mathrm{~m})$ & & Ala4-2 \\
\hline \multicolumn{5}{|l|}{ Pip } \\
\hline Pip-2 & $161.9 ; \mathrm{C} \mathrm{q}$ & & & \\
\hline \multirow[t]{2}{*}{ Pip-3 } & $24.1 ; \mathrm{CH}_{2}$ & $\mathrm{H}_{\mathrm{A}}: 3.34-3.30(\mathrm{~m})$ & & Pip-4- $\mathrm{H}_{\mathrm{B}}$ \\
\hline & & $\mathrm{H}_{\mathrm{B}}: 2.83-2.76(\mathrm{~m})$ & & Pip-4- $\mathrm{H}_{\mathrm{A}} ;$ Pip-4-H \\
\hline \multirow[t]{2}{*}{ Pip-4 } & $29.0 ; \mathrm{CH}_{2}$ & $\mathrm{H}_{\mathrm{A}}: 3.94-3.87(\mathrm{~m})$ & Pip-2; Pip-3; Pip-5; Pip-6; Thz6-4 & Pip-4- $\mathrm{H}_{\mathrm{B}}$ \\
\hline & & $\mathrm{H}_{\mathrm{B}}: 2.21-2.12(\mathrm{~m})$ & Pip-3; Pip-6 & 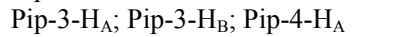 \\
\hline Pip-5 & $57.5 ; \mathrm{C} \mathrm{q}$ & & & \\
\hline Pip-6 & $64.1 ; \mathrm{CH}$ & 5.17 (br s) & Pip-2; Pip-4; Pip-5; Thz13-2; Thz13-3; Thz15-4 & Pip-3- $\mathrm{H}_{\mathrm{B}}$ \\
\hline \multicolumn{5}{|l|}{ Thz6 } \\
\hline Thz6-1 & 161.8; C q & & & \\
\hline Thz6-2 & $146.3 ; \mathrm{C} \mathrm{q}$ & & & \\
\hline Thz6-3 & $124.9 ; \mathrm{CH}$ & $7.98(\mathrm{~s})$ & Thz6-1; Thz6-2; Thz6-4 & \\
\hline Thz6-4 & 169.6; C q & & & \\
\hline \multicolumn{5}{|l|}{ Thr7 } \\
\hline Thr7-1 & $165.5 ; \mathrm{C} \mathrm{q}$ & & & \\
\hline Thr7-2 & $55.8 ; \mathrm{CH}$ & $4.32-4.27(\mathrm{~m})$ & Thr7-3; Thr7-4 & Thr7-3; Thr7-NH \\
\hline Thr7-3 & $66.3 ; \mathrm{CH}$ & $1.52-1.45(\mathrm{~m})$ & Thr7-2 & Thr7-4 \\
\hline Thr7-4 & $18.7 ; \mathrm{CH}_{3}$ & $0.68(\mathrm{~d}, 5.5)$ & Thr7-2; Thr7-3 & Thr7-3 \\
\hline Thr7-NH ${ }^{d}$ & & $7.02-6.94(\mathrm{~m})$ & Thr7-1 & Thr7-2 \\
\hline \multicolumn{5}{|l|}{ Dhb8 } \\
\hline Dhb8-2 & $128.4 ; \mathrm{C} \mathrm{q}$ & & & \\
\hline Dhb8-3 & $132.6 ; \mathrm{CH}$ & $6.08(\mathrm{q}, 6.6)$ & Dhb8-2; Dhb8-4; Tzn9-4 & Dhb8-4 \\
\hline Dhb8-4 & $15.3 ; \mathrm{CH}_{3}$ & $1.48(\mathrm{~d}, 7.8)$ & Dhb8-2; Dhb8-3; Tzn9-4 & Dhb8-3 \\
\hline \multicolumn{5}{|l|}{ Tzn9 } \\
\hline Tzn9-1 & $172.0 ; \mathrm{C} \mathrm{q}$ & & & \\
\hline Tzn9-2 & $78.8 ; \mathrm{CH}$ & $4.84(\mathrm{dd}, 11.8,9.8)$ & Dhb8-2; Tzn9-1; Tzn9-3; Tzn9-4 & Tzn9-3- $\mathrm{H}_{\mathrm{A}}$; Tzn9-3- $\mathrm{H}_{\mathrm{B}}$ \\
\hline \multirow[t]{2}{*}{ Tzn9-3 } & $34.8 ; \mathrm{CH}_{2}$ & $\mathrm{H}_{\mathrm{A}}: 3.54-3.46(\mathrm{~m})$ & Tzn9-2; Tzn9-4 & Tzn9-2; Tzn9-3- $\mathrm{H}_{\mathrm{B}}$ \\
\hline & & $\mathrm{H}_{\mathrm{B}}: 3.03(\mathrm{t}, 12.1)$ & Tzn9-1; Tzn9-2 & Tzn9-2; Tzn9-3- $\mathrm{H}_{\mathrm{A}}$ \\
\hline Tzn9-4 & $170.1 ; \mathrm{C} \mathrm{q}$ & & & \\
\hline \multicolumn{5}{|l|}{ Ile10 } \\
\hline Ile10-2 & $52.9 ; \mathrm{CH}$ & $5.59(\mathrm{~s})$ & Ile10-3; Thz11-4 & Ile10-NH \\
\hline Ile10-3 & $77.2^{\mathrm{c}} ; \mathrm{C} \mathrm{q}$ & & & \\
\hline Ile10-4 & $67.8 ; \mathrm{CH}$ & $3.67(\mathrm{q}, 6.3)$ & Ile10-2; Ile10-3; Ile10-5; Ile10-6 & Ile10-5 \\
\hline Ile10-5 & $15.7 ; \mathrm{CH}_{3}$ & $1.17(\mathrm{~d}, 6.2)$ & Ile10-3; Ile10-4; Ile10-6 & Ile $10-4$ \\
\hline Ile10-6 & $18.5 ; \mathrm{CH}_{3}$ & $1.02(\mathrm{~s})$ & Ile10-2; Ile10-3; Ile10-4 & \\
\hline Ile10-NH ${ }^{\mathrm{d}}$ & & $7.46(\mathrm{~d}, 9.8)$ & & Ile10-2 \\
\hline
\end{tabular}




\begin{tabular}{|c|c|c|c|c|}
\hline Position & $\begin{array}{l}\delta_{\mathrm{C}}[\mathrm{ppm}] \\
\text { mult }\end{array}$ & $\begin{array}{l}\delta_{\mathrm{H}}[\mathrm{ppm}] ; \\
\text { (mult, } \mathrm{J} \text { in } \mathrm{Hz} \text { ) }\end{array}$ & НМBC $^{\mathbf{a}}$ & $\operatorname{cosY}^{b}$ \\
\hline \multicolumn{5}{|l|}{ Thz11 } \\
\hline $\begin{array}{l}\text { Thz11-1 } \\
\text { Thz11-2 } \\
\text { Thz11-3 } \\
\text { Thz11-4 }\end{array}$ & $\begin{array}{l}162.4 ; \mathrm{C} \mathrm{q} \\
150.0 ; \mathrm{C} \mathrm{q} \\
125.5 ; \mathrm{CH} \\
166.2 ; \mathrm{C} \mathrm{q}\end{array}$ & $8.13(\mathrm{~s})$ & Thz11-1; Thz11-2; Thz11-4 & \\
\hline $\begin{array}{l}\text { Thr12 } \\
\text { Thr12-2 } \\
\text { Thr12-3 } \\
\text { Thr12-4 } \\
\text { Thr12-NH }\end{array}$ & $\begin{array}{l}55.5 ; \mathrm{CH} \\
71.9 ; \mathrm{CH} \\
18.7 ; \mathrm{CH}_{3}\end{array}$ & $\begin{array}{l}5.62(\mathrm{~s}) \\
6.17(\mathrm{q}, 6.2) \\
1.49(\mathrm{~d}, 7.4) \\
8.65(\mathrm{~d}, 9.1)\end{array}$ & $\begin{array}{l}\text { Thr12-3; Thr12-4; Thz13-2; Thz13-4 } \\
\text { Thr12-4; Thz13-4; Q-1 } \\
\text { Thr12-2; Thr12-3 }\end{array}$ & $\begin{array}{l}\text { Thr12-3; Thr12-NH } \\
\text { Thr12-4 } \\
\text { Thr12-3 } \\
\text { Thr12-2 }\end{array}$ \\
\hline $\begin{array}{l}\text { Thz13 } \\
\text { Thz13-2 } \\
\text { Thz13-3 } \\
\text { Thz13-4 }\end{array}$ & $\begin{array}{l}157.0 ; \mathrm{C} \mathrm{q} \\
118.3 ; \mathrm{CH} \\
170.1 ; \mathrm{C} \mathrm{q}\end{array}$ & $7.43(\mathrm{~s})$ & Pip-6; Thz13-2; Thz13-4 & \\
\hline $\begin{array}{l}\text { Thz15 } \\
\text { Thz15-1 } \\
\text { Thz15-2 } \\
\text { Thz15-3 } \\
\text { Thz15-4 }\end{array}$ & $\begin{array}{l}159.6 ; \mathrm{C} \mathrm{q} \\
149.8 ; \mathrm{C} \mathrm{q} \\
127.6 ; \mathrm{CH} \\
168.3 ; \mathrm{C} \mathrm{q}\end{array}$ & $8.15(\mathrm{~s})$ & Thz15-1; Thz15-2; Thz15-4 & \\
\hline $\begin{array}{l}\text { Dha16 } \\
\text { Dha16-1 } \\
\text { Dha16-2 } \\
\text { Dha16-3 }\end{array}$ & $\begin{array}{l}162.0 ; \mathrm{C} \mathrm{q} \\
134.0 ; \mathrm{C} \mathrm{q} \\
103.2 ; \mathrm{CH}_{2}\end{array}$ & $\begin{array}{l}\mathrm{H}_{\mathrm{A}}: 6.56(\mathrm{~s}) \\
\mathrm{H}_{\mathrm{B}}: 5.47(\mathrm{~s})\end{array}$ & $\begin{array}{l}\text { Dha16-1; Dha16-2 } \\
\text { Dha16-1; Dha16-2 }\end{array}$ & $\begin{array}{l}\text { Dha16-3- } \mathrm{H}_{\mathrm{B}} \\
\text { Dha16-3- } \mathrm{H}_{\mathrm{A}}\end{array}$ \\
\hline \multicolumn{5}{|l|}{ Dha17 } \\
\hline $\begin{array}{l}\text { Dha17-1 } \\
\text { Dha17-2 } \\
\text { Dha17-3 }\end{array}$ & $\begin{array}{l}166.0 ; \mathrm{C} \mathrm{q} \\
132.8 ; \mathrm{C} \mathrm{q} \\
104.3 ; \mathrm{CH}_{2}\end{array}$ & $\begin{array}{l}\mathrm{H}_{\mathrm{A}}: 6.39(\mathrm{~s}) \\
\mathrm{H}_{\mathrm{B}}: 5.55(\mathrm{~s})\end{array}$ & $\begin{array}{l}\text { Dha17-1; Dha17-2 } \\
\text { Dha17-1; Dha17-2 }\end{array}$ & $\begin{array}{l}\text { Dha17-3- } \mathrm{H}_{\mathrm{B}} \\
\text { Dha17-3- } \mathrm{H}_{\mathrm{A}}\end{array}$ \\
\hline \multicolumn{5}{|r|}{ 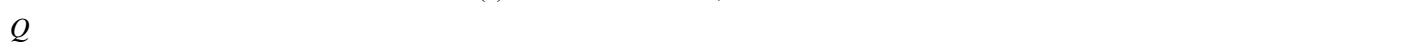 } \\
\hline $\begin{array}{l}\text { Q-1 } \\
\text { Q-2 } \\
\text { Q-3 } \\
\text { Q-4 } \\
\text { Q-5 }\end{array}$ & $\begin{array}{l}160.8 ; \mathrm{C} \mathrm{q} \\
143.5 ; \mathrm{C} \mathrm{q} \\
122.4 ; \mathrm{CH} \\
153.2 ; \mathrm{C} \mathrm{q} \\
127.3 ; \mathrm{C} \mathrm{q}\end{array}$ & $7.16(\mathrm{~s})$ & Q-1; Q-5; Q-11 & \\
\hline Q-6 & 123.3; $\mathrm{CH}$ & $6.76(d, 9.9)$ & Q-5; Q-8; Q-10 & Q-7 \\
\hline Q-7 & $129.6 ; \mathrm{CH}$ & $6.25(\mathrm{dd}, 9.0,5.8)$ & Q-5; Q-8; Q-9 & Q-6; Q-8; Q-9 \\
\hline Q-8 & $59.2 ; \mathrm{CH}$ & $3.54-3.46(\mathrm{~m})$ & Ile1-2; Q-6; Q-7; Q-9; Q-10 & Q-7; Q-9 \\
\hline $\begin{array}{l}\text { Q-9 } \\
\text { Q-10 }\end{array}$ & $\begin{array}{l}67.7 ; \mathrm{CH} \\
154.3 ; \mathrm{C} \mathrm{q}\end{array}$ & $4.36(\mathrm{~s})$ & Q-5; Q-7; Q-8; Q-10 & Q-7; Q-8 \\
\hline Q-11 & $64.4 ; \mathrm{CH}$ & $5.17(\mathrm{br} \mathrm{s})$ & Q-3; Q-12 & Q-12 \\
\hline Q-12 & $22.5 ; \mathrm{CH}_{3}$ & $1.24(\mathrm{~d}, 6.4)$ & Q-4; Q-11 & Q-11 \\
\hline
\end{tabular}

${ }^{\mathrm{a}} \mathrm{HMBC}$ correlations are from the proton to the indicated carbon.

${ }^{\mathrm{b}} \mathrm{COSY}$ correlations are from the proton to the proton attached to the indicated position.

${ }^{c}$ The $\delta$ of this resonance was determined by HMBC due to overlap with the $\mathrm{CDCl}_{3}$ resonance.

${ }^{\mathrm{d}}$ Only those amide resonances demonstrating either HMBC or COSY correlations to neighboring carbons or protons, respectively, were assigned. 
Figure S18. MS analysis of thiostrepton Ala2Ile- $\Delta$ Ile1 isolated from S. laurentii NDS1/int-A2I.

(A) HPLC-MS analysis. (1) Chromatogram extracted for $\mathrm{m} / \mathrm{z} 797.2$, the calculated $[\mathrm{M}+2 \mathrm{H}]^{2+}$ ion of thiostrepton Ala2Ile- $\Delta$ Ile1. (2) Total ion chromatogram.

(1) Zhang_121217_02

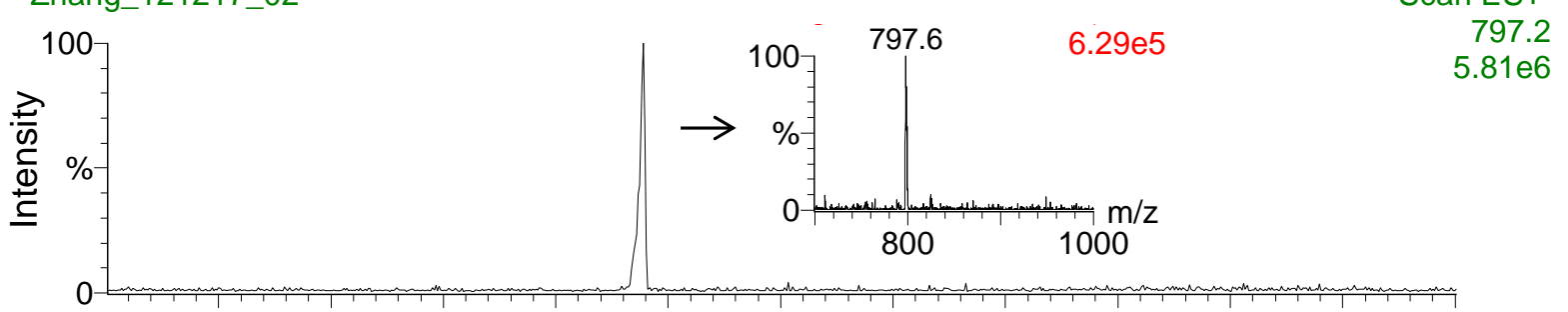

(2) Zhang_121217_02

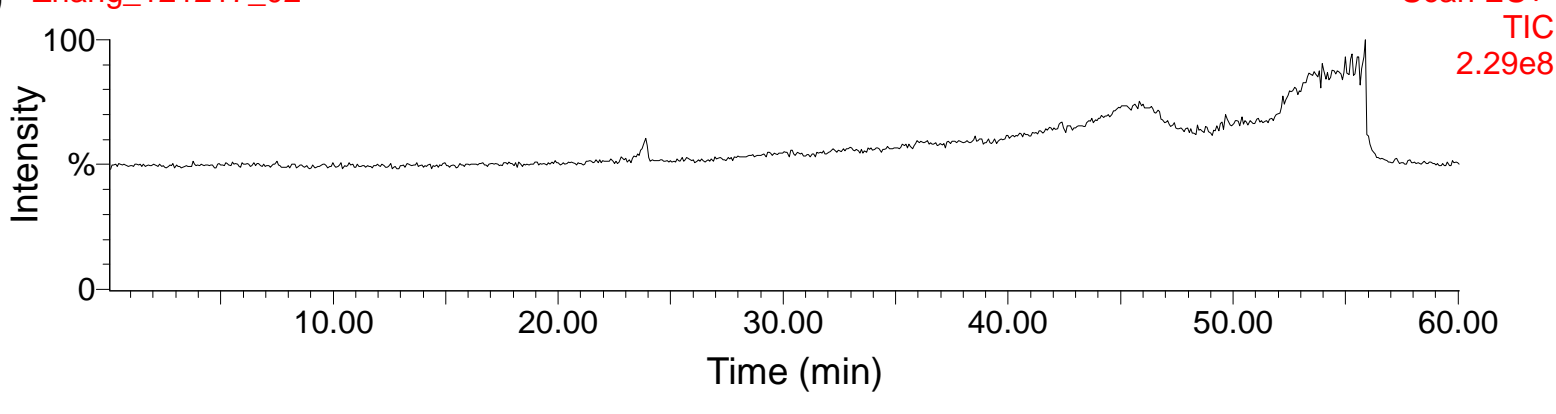

(B) MALDI MS spectrum of thiostrepton Ala2Ile- $\Delta$ Ile1.

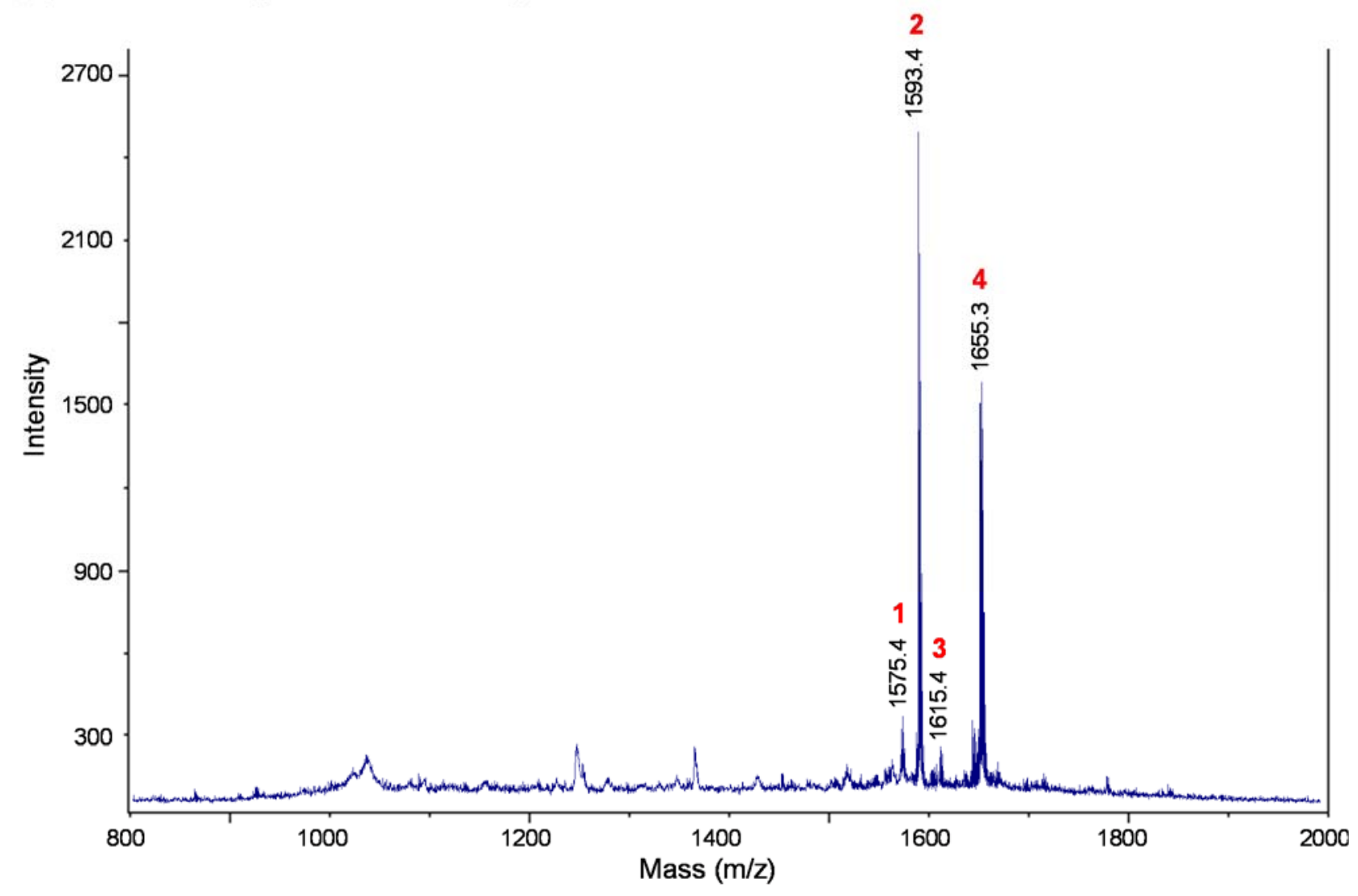


(C) MALDI MS/MS of parent ion $m / z$ 1593.4.

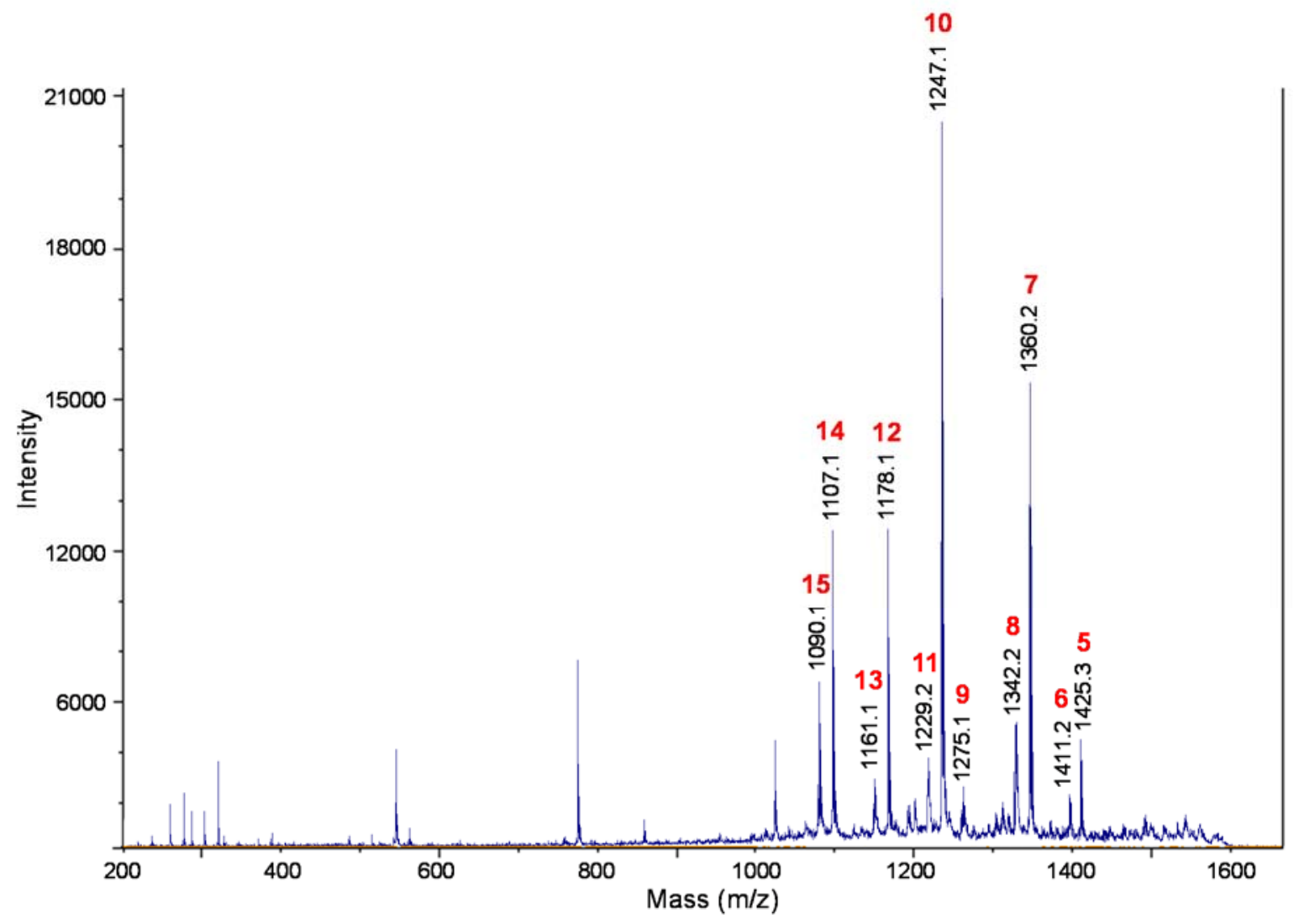

(D) Table and structure showing key ions and fragments in the MALDI MS and MS/MS of thiostrepton Ala2Ile- $\Delta$ Ile1.

\begin{tabular}{|c|c|c|}
\hline Fragment & Expected & Observed \\
\hline 1. $\mathrm{M}-\mathrm{H}_{2} \mathrm{O}+\mathrm{H}^{+}$ & 1575.5 & 1575.4 \\
\hline 2. $\mathrm{M}+\mathrm{H}^{+}$(Parent ion) & 1593.5 & 1593.4 \\
\hline 3. $\mathrm{M}+\mathrm{Na}^{+}$ & 1615.4 & 1615.4 \\
\hline 4. $\mathrm{M}+\mathrm{Cu}^{+}$ & 1655.4 & 1655.3 \\
\hline 5. M-Dhb8-Tzn9+H ${ }^{+}$ & 1425.4 & 1425.3 \\
\hline 6. M-Ile2-Dha3+H ${ }^{+}$ & 1411.4 & 1411.2 \\
\hline 7. $M-Q A+H^{+}$ & 1360.4 & 1360.2 \\
\hline 8. M-QA- $\mathrm{H}_{2} \mathrm{O}+\mathrm{H}^{+}$ & 1342.4 & 1342.2 \\
\hline 9. M-QA-(lle2-CO)+H+ & 1275.3 & 1275.1 \\
\hline 10. M-QA-Ile2+H ${ }^{+}$ & 1247.3 & 1247.1 \\
\hline 11. M-QA-Ile2- $\mathrm{H}_{2} \mathrm{O}+\mathrm{H}^{+}$ & 1229.3 & 1229.2 \\
\hline 12. M-QA-Ile2-Dha3+H+ & 1178.3 & 1178.1 \\
\hline 13. M-QA-Ile2-Dha3-OH+H ${ }^{+}$ & 1161.3 & 1161.1 \\
\hline 14. M-QA-Ile2-Dha3-Ala4+H' & 1107.3 & 1107.1 \\
\hline 15. M-QA-lle2-Dha3-Ala4-OH+H+ & 1090.2 & 1090.1 \\
\hline
\end{tabular}

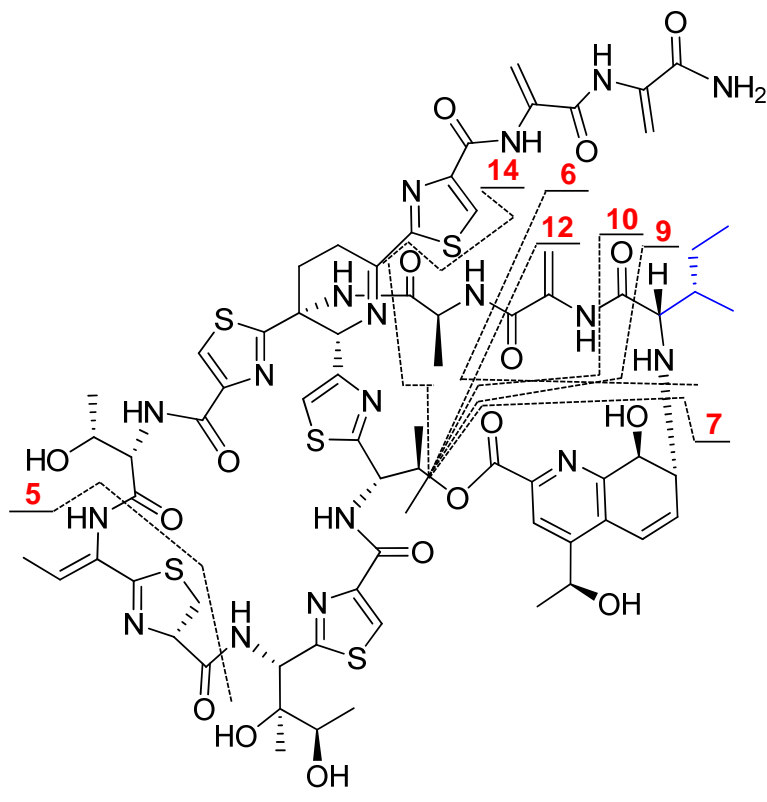


Figure S19. Structure and numbering system used for thiostrepton Ala2Ile- $\Delta$ Ile1.

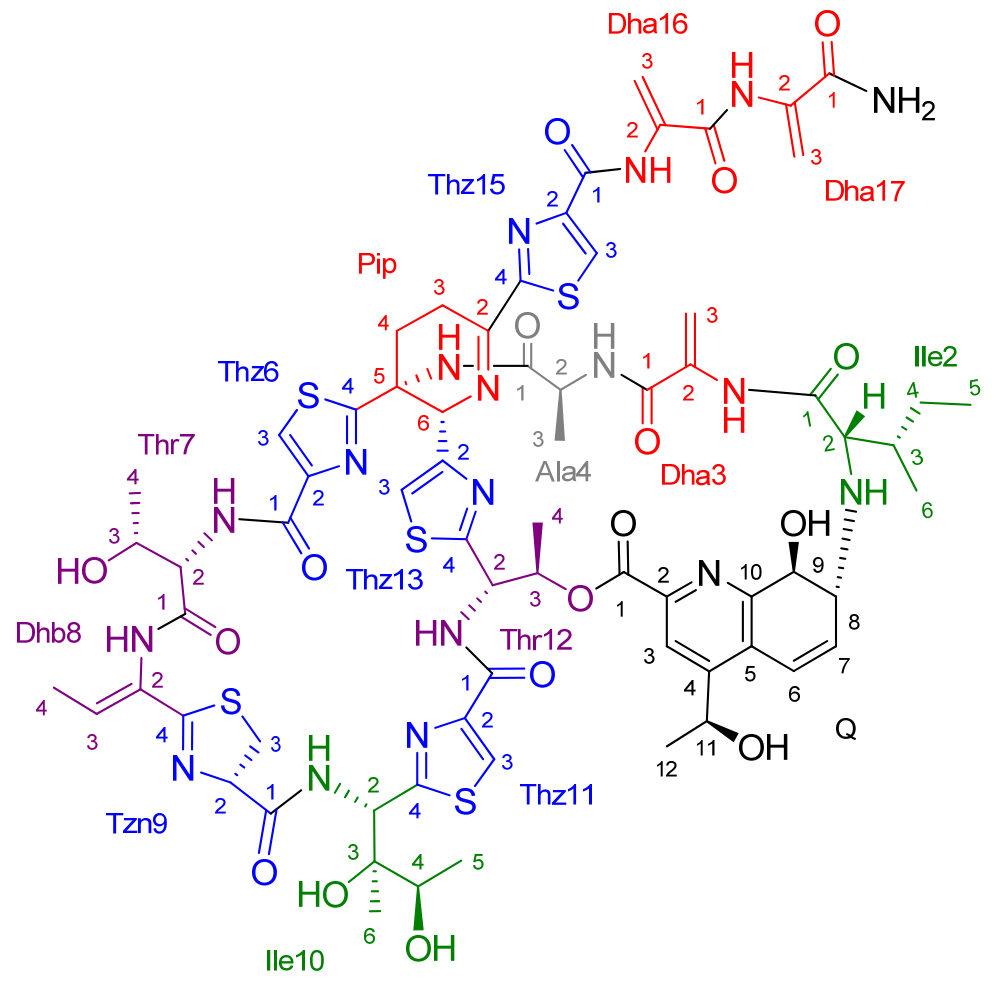




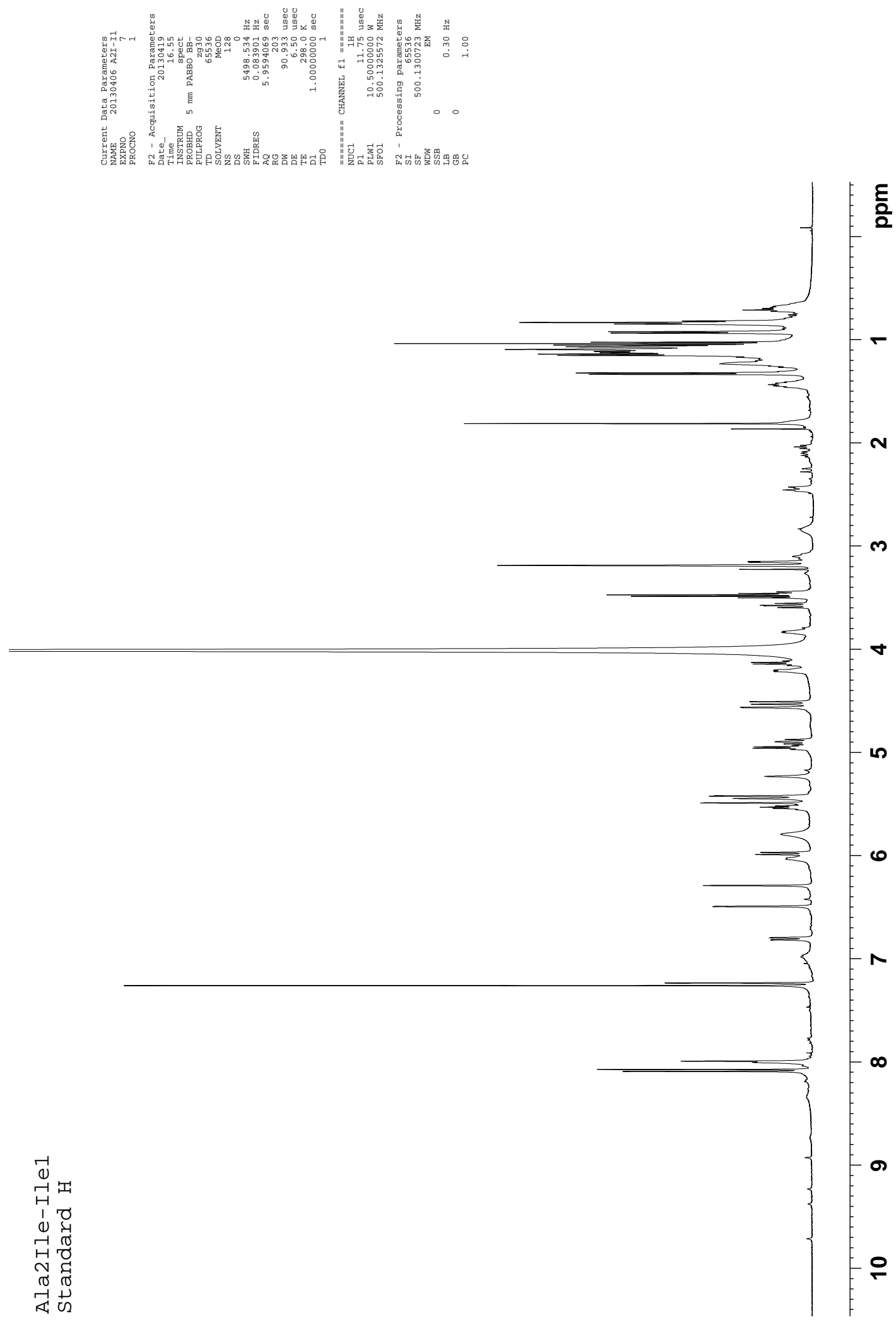

Figure S20. ${ }^{1} \mathrm{H}$ NMR spectrum of thiostrepton Ala2Ile- $\Delta \mathrm{Ile} 1\left(500 \mathrm{MHz}, \mathrm{CDCl}_{3}-\mathrm{CD}_{3} \mathrm{OD}\right.$ 4:1, $25^{\circ} \mathrm{C}$ ). 


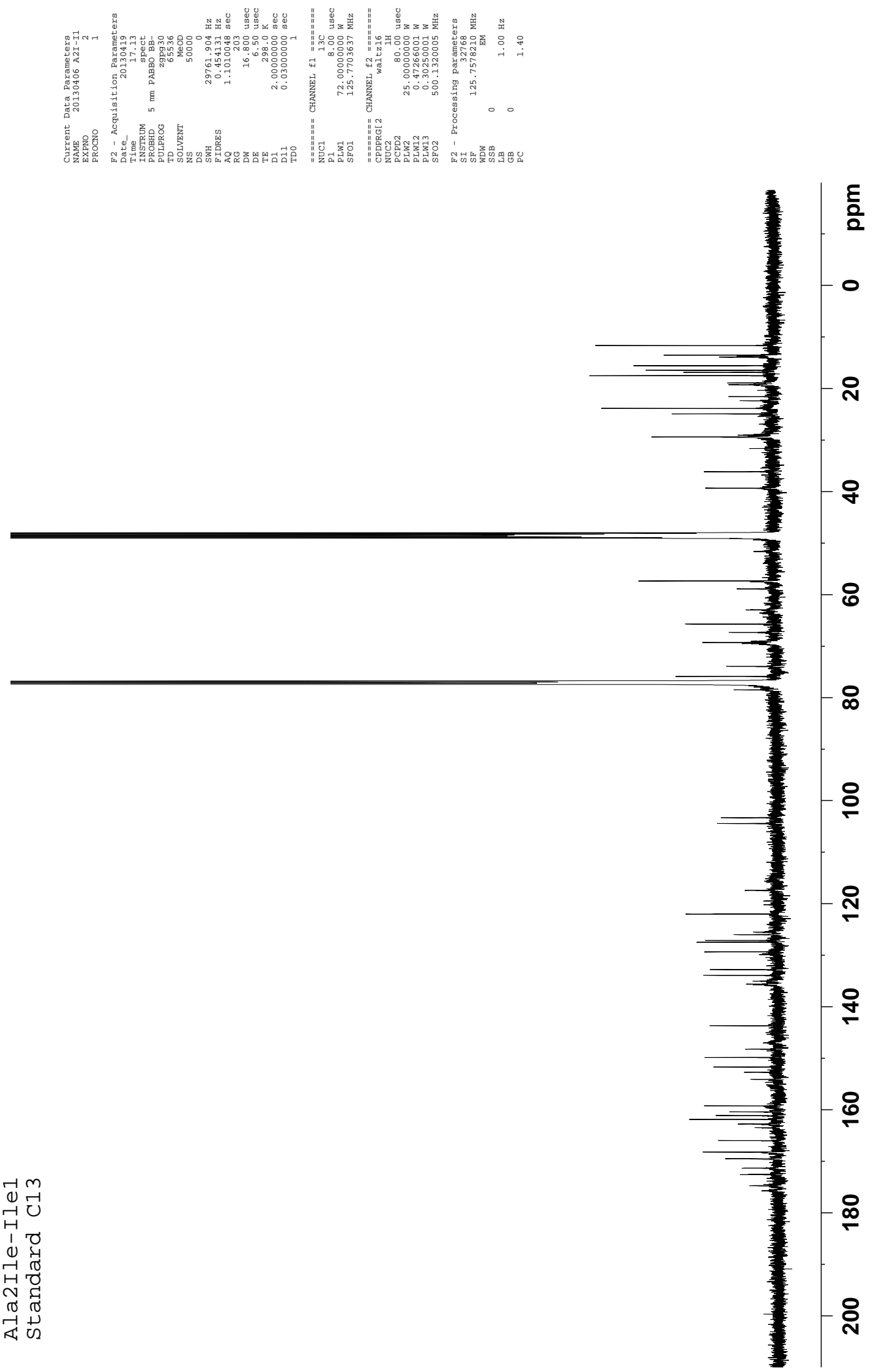

Figure S21. ${ }^{13} \mathrm{C}$ NMR spectrum of thiostrepton Ala2Ile- $\Delta \mathrm{Ile} 1\left(125 \mathrm{MHz}, \mathrm{CDCl}_{3}-\mathrm{CD}_{3} \mathrm{OD}\right.$ 4:1, $\left.25^{\circ} \mathrm{C}\right)$. 

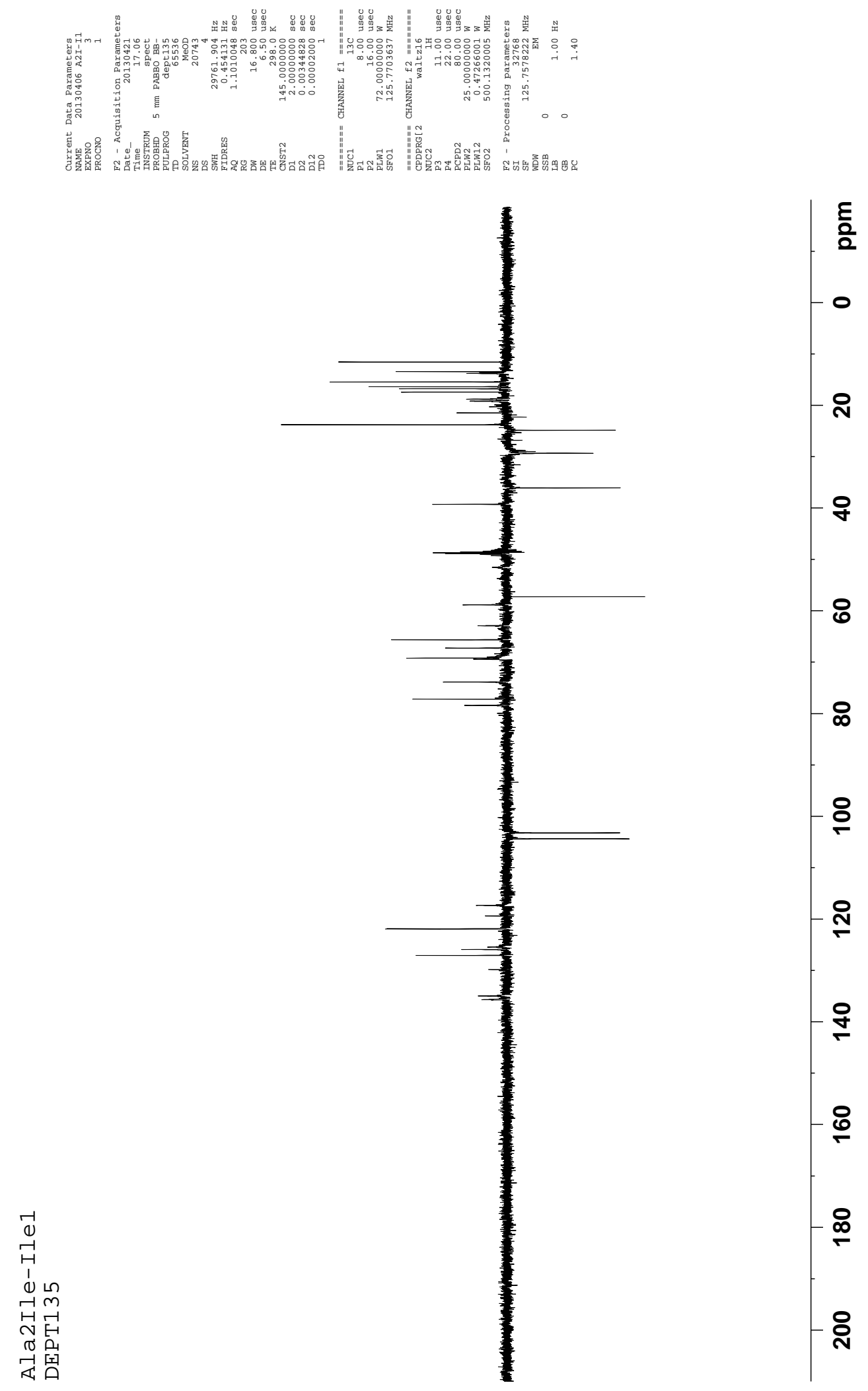

Figure S22. DEPT-135 NMR spectrum of thiostrepton Ala2Ile- $\Delta$ Ile1 $\left(125 \mathrm{MHz}, \mathrm{CDCl}_{3}-\mathrm{CD}_{3} \mathrm{OD}\right.$ $\left.4: 1,25^{\circ} \mathrm{C}\right)$. 


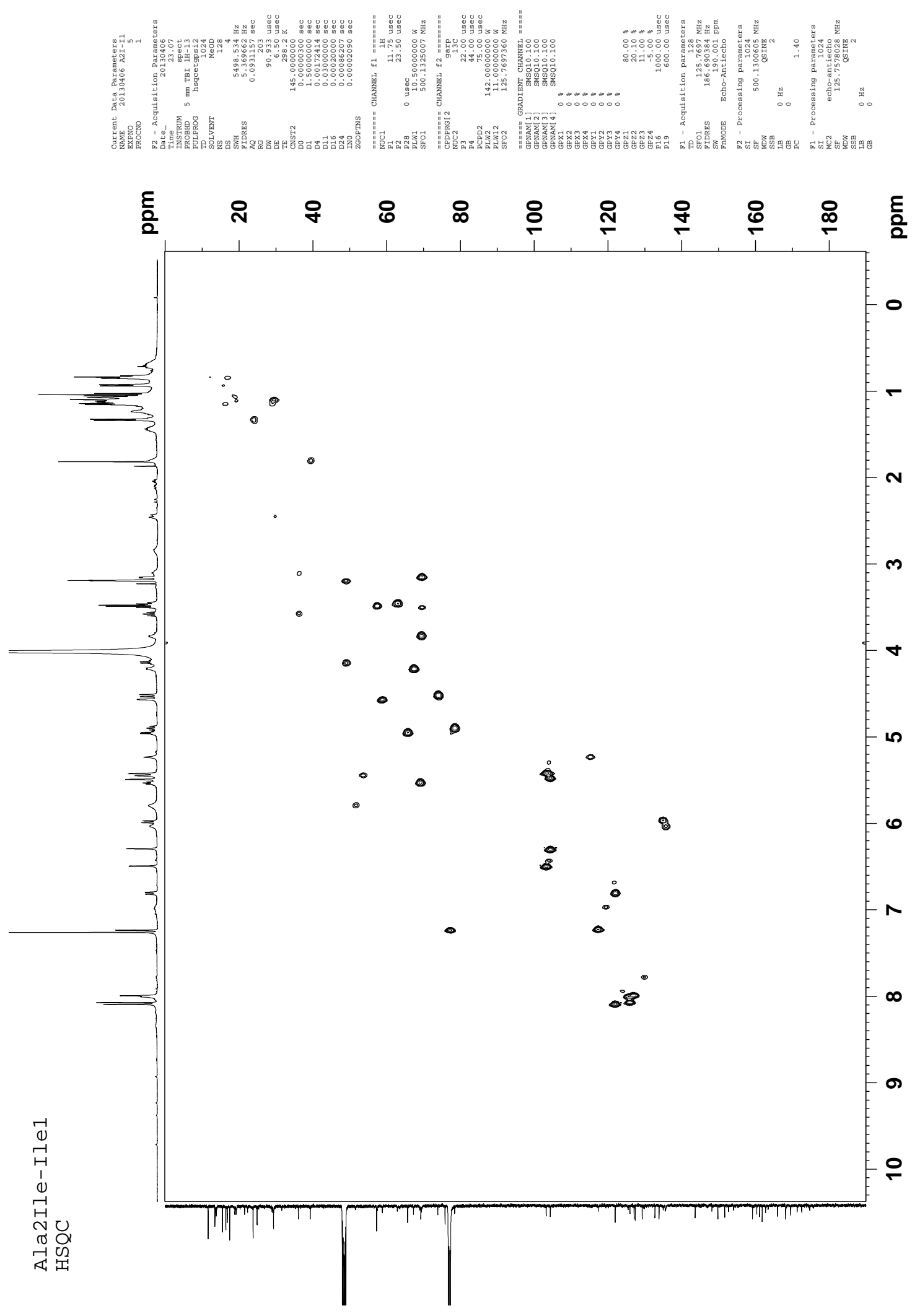

Figure S23. gHSQC NMR spectrum of thiostrepton Ala2Ile- $\Delta$ Ile1 $\left(500 \mathrm{MHz}, \mathrm{CDCl}_{3}-\mathrm{CD}_{3} \mathrm{OD} 4: 1\right.$, $25^{\circ} \mathrm{C}$ ). 


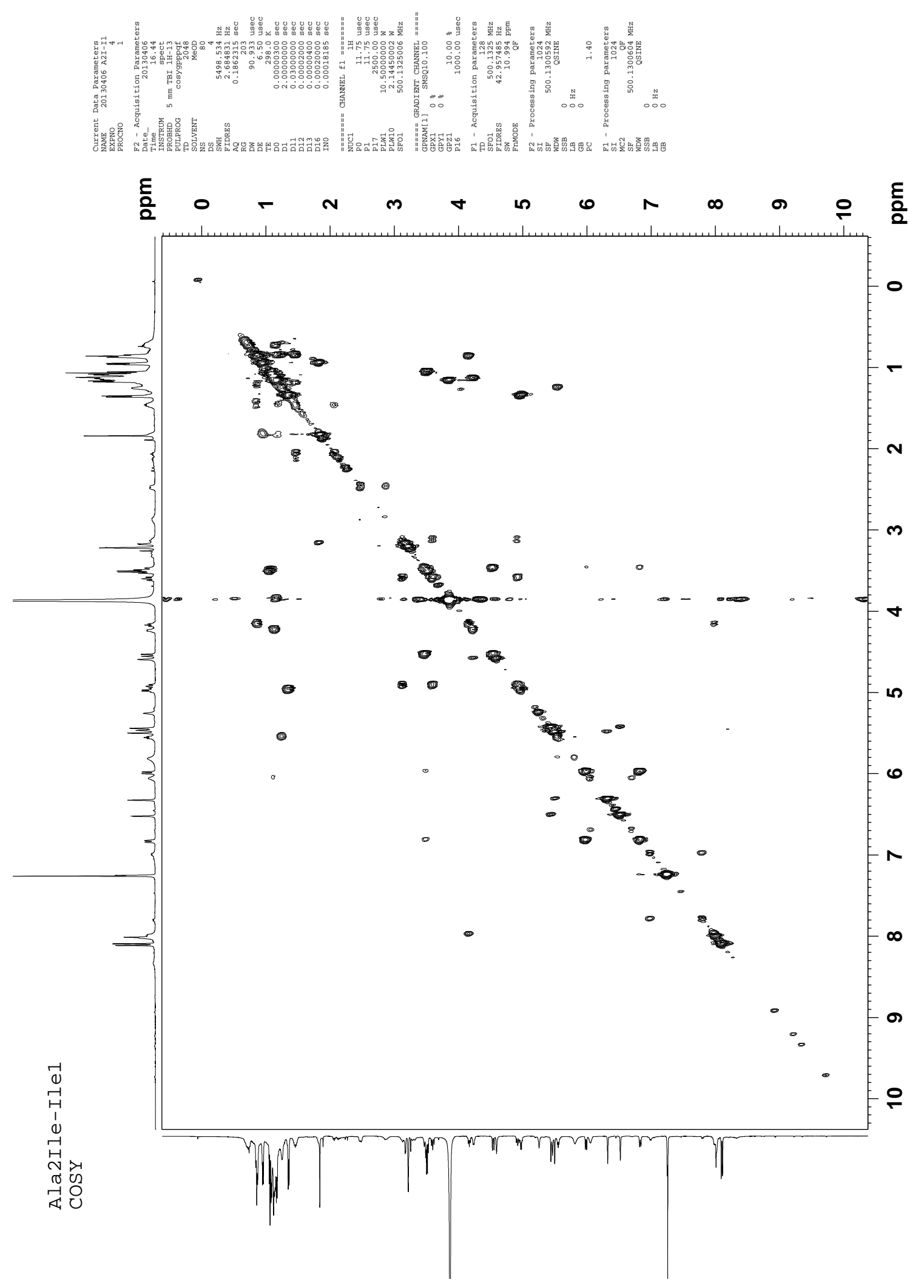

Figure S24. gCOSY NMR spectrum of thiostrepton Ala2Ile- $\Delta \mathrm{Ile} 1\left(125 \mathrm{MHz}, \mathrm{CDCl}_{3}-\mathrm{CD}_{3} \mathrm{OD}\right.$ 4:1, $\left.25^{\circ} \mathrm{C}\right)$. 


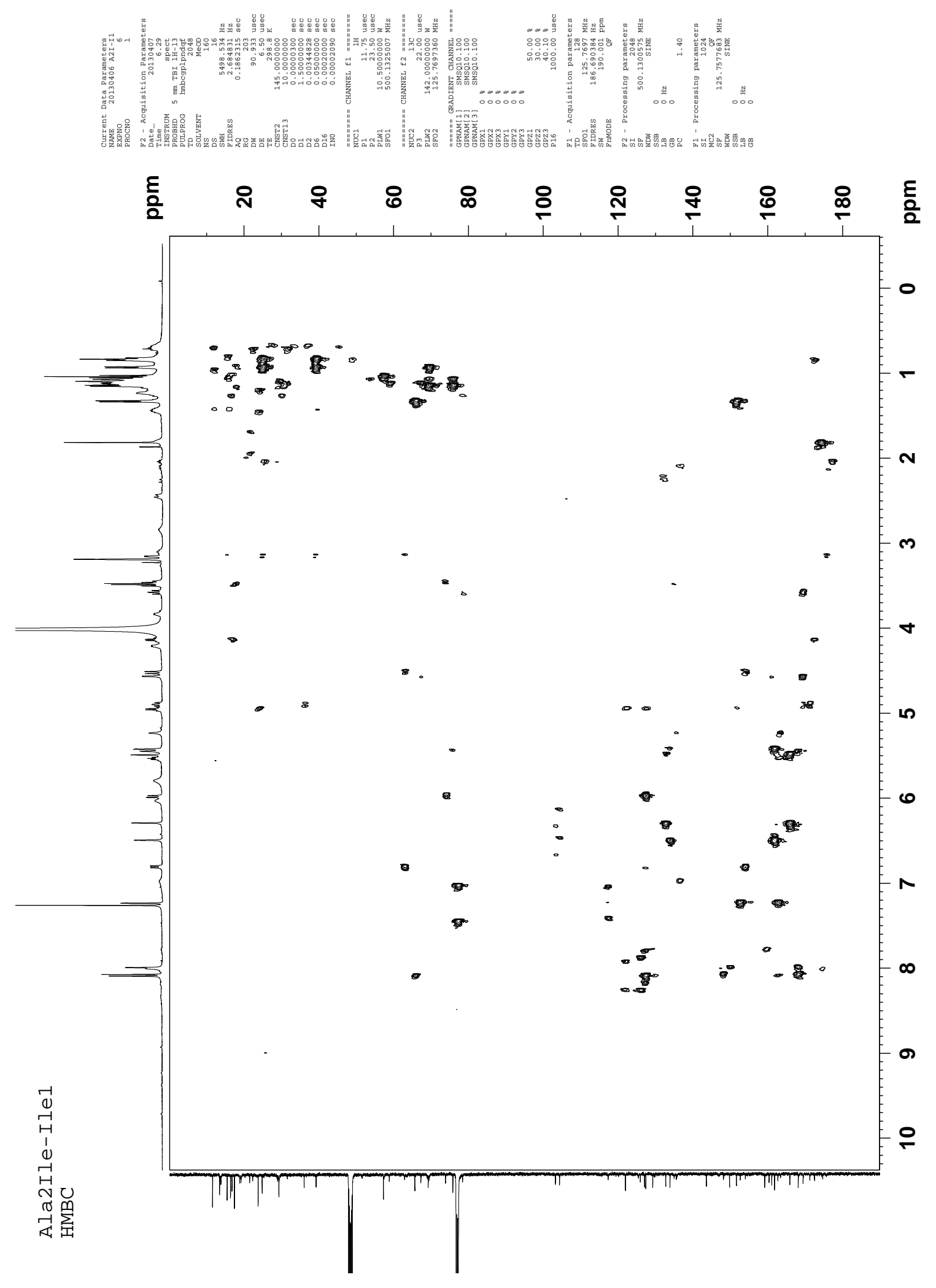

Figure S25. gHMBC NMR spectrum of thiostrepton Ala2Ile- $\Delta$ Ile1 (125 MHz, CDCl3-CD 3 OD 4:1, $25^{\circ} \mathrm{C}$ ). 
Table S3. ${ }^{1} \mathrm{H}$ and ${ }^{13} \mathrm{C}$ NMR assignments of thiostrepton Ala2Ile- $\Delta$ Ile1

\begin{tabular}{|c|c|c|c|c|}
\hline Position & $\begin{array}{l}\delta_{\mathrm{C}}[\mathrm{ppm}] ; \\
\text { mult }\end{array}$ & $\begin{array}{l}\delta_{\mathrm{H}}[\mathrm{ppm}] ; \\
\text { (mult, } \mathrm{J} \text { in } \mathrm{Hz})\end{array}$ & HMBC $^{\mathbf{a}}$ & $\operatorname{COSY}^{b}$ \\
\hline \multicolumn{5}{|l|}{ Ile2 } \\
\hline Ile2-1 & 174.7; C q & & & \\
\hline Ile2-2 & $69.4 ; \mathrm{CH}$ & $3.15(\mathrm{~d}, 3.8)$ & Ile2-3; Ile2-4; Ile2-6 & Ile2-3 \\
\hline Ile2-3 & $39.3 ; \mathrm{CH}$ & $1.82-1.78(\mathrm{~m})$ & Ile2-1 & Ile2-2; Ile2-4-H $\mathrm{H}_{\mathrm{A}}$; Ile2-4-H $\mathrm{H}_{\mathrm{B}}$; Ile2-6 \\
\hline \multirow[t]{2}{*}{ Ile2-4 } & $24.9 ; \mathrm{CH}_{2}$ & $\mathrm{H}_{\mathrm{A}}: 1.48-1.39(\mathrm{~m})$ & Ile2-3; Ile2-5; Ile2-6 & Ile2-3; Ile2-4- $\mathrm{H}_{\mathrm{B}} ;$ Ile2-5 \\
\hline & & $\mathrm{H}_{\mathrm{B}}: 1.21-1.16(\mathrm{~m})$ & Ile2-2 & Ile2-3; Ile2-4-H $\mathrm{H}_{\mathrm{A}}$; Ile2-5 \\
\hline Ile2-5 & $11.6 ; \mathrm{CH}_{3}$ & $0.83(\mathrm{t}, 7.3)$ & Ile2-3; Ile2-4; Ile2-6 & Ile2-4- $\mathrm{H}_{\mathrm{A}} ; \mathrm{Ile} 2-4-\mathrm{H}_{\mathrm{B}}$ \\
\hline Ile2-6 & $15.5 ; \mathrm{CH}_{3}$ & $0.93(\mathrm{~d}, 6.9)$ & Ile2-2; Ile2-3; Ile2-4; Ile2-5 & Ile2-3 \\
\hline \multicolumn{5}{|l|}{ Dha3 } \\
\hline Dha3-1 & $163.4 ; \mathrm{C} \mathrm{q}$ & & & \\
\hline Dha3-2 & 135.6; $\mathrm{C} \mathrm{q}$ & & & \\
\hline \multirow[t]{2}{*}{ Dha3-3 } & $115.2 ; \mathrm{CH}_{2}$ & $\mathrm{H}_{\mathrm{A}}: 5.79($ br s $)$ & & \\
\hline & & $\mathrm{H}_{\mathrm{B}}: 5.23(\mathrm{br} \mathrm{s})$ & Dha3-1; Dha3-2 & \\
\hline \multicolumn{5}{|l|}{ Ala4 } \\
\hline Ala4-1 & $172.5 ; \mathrm{C} \mathrm{q}$ & & & \\
\hline Ala4-2 & $49.3 ; \mathrm{CH}$ & $4.14(\mathrm{q}, 7.4)$ & Ala4-1; Ala4-3 & Ala4-3 \\
\hline Ala4-3 & $16.8 ; \mathrm{CH}_{3}$ & $0.84(\mathrm{~d}, 7.3)$ & Ala4-1; Ala4-2 & Ala4-2 \\
\hline \multicolumn{5}{|l|}{ Pip } \\
\hline Pip-2 & 159.2; C q & & & \\
\hline \multirow[t]{2}{*}{ Pip-3 } & $22.3 ; \mathrm{CH}_{2}$ & $\mathrm{H}_{\mathrm{A}}: 3.50-3.40(\mathrm{~m})$ & & Pip-3- $\mathrm{H}_{\mathrm{B}} ;$ Pip-4- $\mathrm{H}_{\mathrm{B}}$ \\
\hline & & $\mathrm{H}_{\mathrm{B}}: 2.89-2.80(\mathrm{~m})$ & & Pip-4- $\mathrm{H}_{\mathrm{B}}$ \\
\hline \multirow[t]{2}{*}{ Pip-4 } & $29.3 ; \mathrm{CH}_{2}$ & $\mathrm{H}_{\mathrm{A}}: 3.86-3.80(\mathrm{~m})$ & & \\
\hline & & $\mathrm{H}_{\mathrm{B}}: 2.48-2.42(\mathrm{~m})$ & & Pip-3- $\mathrm{H}_{\mathrm{A}} ;$ Pip-3- $\mathrm{H}_{\mathrm{B}}$ \\
\hline Pip-5 & $57.5 ; \mathrm{C} \mathrm{q}$ & & & \\
\hline Pip-6 & $63.6 ; \mathrm{CH}$ & 5.23 (br s) & & \\
\hline \multicolumn{5}{|l|}{ Thz6 } \\
\hline Thz6-1 & 161.1; C q & & & \\
\hline Thz6-2 & $149.8 ; \mathrm{C} \mathrm{q}$ & & & \\
\hline Thz6-3 & 127.1; $\mathrm{CH}$ & $7.99(\mathrm{~s})$ & Thz6-2; Thz6-4 & \\
\hline Thz6-4 & $168.2 ; \mathrm{C} \mathrm{q}$ & & & \\
\hline \multicolumn{5}{|l|}{ Thr7 } \\
\hline Thr7-1 & 169.5; C q & & & \\
\hline Thr7-2 & $58.8 ; \mathrm{CH}^{1}$ & $4.56(\mathrm{~d}, 2.0)$ & Thz6-1; Thr7-1; Thr7-3 & Thr7-3 \\
\hline Thr7-3 & $67.3 ; \mathrm{CH}$ & $4.23-4.18(\mathrm{~m})$ & & Thr7-2; Thr7-4 \\
\hline Thr7-4 & $19.2 ; \mathrm{CH}_{3}$ & $1.12(\mathrm{~d}, 6.0)$ & Thr7-2; Thr7-3 & Thr7-3 \\
\hline \multicolumn{5}{|l|}{ Dhb8 } \\
\hline Dhb8-2 & 127.4; C q & & & \\
\hline Dhb8-3 & $135.7 ; \mathrm{CH}$ & $6.08-6.03(\mathrm{~m})$ & Dhb8-2 & Dhb8-4 \\
\hline Dhb8-4 & $19.2 ; \mathrm{CH}_{3}$ & $1.13-1.09(\mathrm{~m})$ & & \\
\hline \multicolumn{5}{|l|}{ Tzn9 } \\
\hline Tzn9-1 & 171.3; $\mathrm{C} \mathrm{q}$ & & & \\
\hline Tzn9-2 & $78.4 ; \mathrm{CH}$ & $4.90(\mathrm{dd}, 11.4,9.8)$ & Tzn9-1; Tzn9-3; Tzn9-4 & Tzn9-3- $\mathrm{H}_{\mathrm{A}} ; \mathrm{Tzn} 9-3-\mathrm{H}_{\mathrm{B}}$ \\
\hline \multirow[t]{2}{*}{ Tzn9-3 } & $36.1 ; \mathrm{CH}_{2}$ & $\mathrm{H}_{\mathrm{A}}: 3.58(\mathrm{dd}, 11.1,9.2)$ & Tzn9-2; Tzn9-4 & Tzn9-2; Tzn9-3- $\mathrm{H}_{\mathrm{B}}$ \\
\hline & & $\mathrm{H}_{\mathrm{B}}: 3.10(\mathrm{t}, 12.0)$ & & Tzn9-2; Tzn9-3- $\mathrm{H}_{\mathrm{A}}$ \\
\hline Tzn9-4 & $169.5 ; \mathrm{C} \mathrm{q}$ & & & \\
\hline \multicolumn{5}{|l|}{ Ile10 } \\
\hline Ile10-2 & 53.7; CH & $5.45(\mathrm{~s})$ & Ile10-3; Thz11-4 & \\
\hline Ile10-3 & $75.8 ; \mathrm{C} \mathrm{q}$ & & & \\
\hline Ile10-4 & $69.3 ; \mathrm{CH}$ & $3.86-3.80(\mathrm{~m})$ & & Ile $10-5$ \\
\hline Ile10-5 & $16.4 ; \mathrm{CH}_{3}$ & $1.15(\mathrm{~d}, 6.5)$ & Ile10-3; Ile10-4 & Ile10-4 \\
\hline Ile10-6 & $18.8 ; \mathrm{CH}_{3}$ & $1.07(\mathrm{~s})$ & Ile10-2; Ile10-3; Ile10-4; Ile10-5 & \\
\hline \multicolumn{5}{|l|}{ Thz11 } \\
\hline Thz11-1 & 162.7; C q & & & \\
\hline Thz11-2 & $149.8 ; \mathrm{C} \mathrm{q}$ & & & \\
\hline Thz11-3 & $121.9 ; \mathrm{CH}$ & $8.09(\mathrm{~s})$ & Thz11-1; Thz11-4 & \\
\hline Thz11-4 & $168.3 ; \mathrm{C} \mathrm{q}$ & & & \\
\hline \multicolumn{5}{|l|}{ Thr12 } \\
\hline Thr12-2 & $51.6 ; \mathrm{CH}$ & 5.79 (br s) & & Thr12-3 \\
\hline Thr12-3 & 69.3; CH & 5.53 (pentet, 6.1 ) & Q-1 & Thr12-2; Thr12-4 \\
\hline Thr12-4 & $13.8 ; \mathrm{CH}_{3}$ & $1.26-1.21(\mathrm{~m})$ & & Thr12-3 \\
\hline
\end{tabular}




\begin{tabular}{|c|c|c|c|c|}
\hline Position & $\begin{array}{l}\delta_{\mathrm{C}}[\mathrm{ppm}] ; \\
\text { mult }\end{array}$ & $\begin{array}{l}\delta_{\mathrm{H}}[\mathrm{ppm}] ; \\
\text { (mult, J in Hz) }\end{array}$ & HMBC $^{\text {a }}$ & $\cos ^{b}$ \\
\hline \multicolumn{5}{|l|}{ Thz13 } \\
\hline Thz13-2 & $159.2 ; \mathrm{C} \mathrm{q}$ & & & \\
\hline Thz13-3 & $125.5 ; \mathrm{CH}$ & $8.00(\mathrm{~s})$ & Thz13-4 & \\
\hline Thz13-4 & 168.2; $\mathrm{C} \mathrm{q}$ & & & \\
\hline \multicolumn{5}{|l|}{ Thz15 } \\
\hline Thz15-1 & $160.4 ; \mathrm{C} \mathrm{q}$ & & & \\
\hline Thz15-2 & $148.2 ; \mathrm{C} \mathrm{q}$ & & & \\
\hline Thz15-3 & 126.0; $\mathrm{CH}$ & $8.07(\mathrm{~s})$ & Thz15-2; Thz15-4 & \\
\hline Thz15-4 & $168.3 ; \mathrm{C} \mathrm{q}$ & & & \\
\hline \multicolumn{5}{|l|}{ Dha16 } \\
\hline Dha16-1 & 161.8; C q & & & \\
\hline Dha16-2 & $133.9 ; \mathrm{C} \mathrm{q}$ & & & \\
\hline \multirow[t]{2}{*}{ Dha16-3 } & $103.2 ; \mathrm{CH}_{2}$ & $\mathrm{H}_{\mathrm{A}}: 6.49(\mathrm{~d}, 2.2)$ & Dha16-1; Dha16-2 & Dha16-3- $\mathrm{H}_{\mathrm{B}}$ \\
\hline & & $\mathrm{H}_{\mathrm{B}}: 5.42(\mathrm{~d}, 2.1)$ & Dha16-1; Dha16-2 & Dha16-3- $\mathrm{H}_{\mathrm{A}}$ \\
\hline \multicolumn{5}{|l|}{ Dha17 } \\
\hline Dha17-1 & $165.9 ; \mathrm{Cq}$ & & & \\
\hline Dha17-2 & $132.7 ; \mathrm{C} \mathrm{q}$ & & & \\
\hline \multirow[t]{2}{*}{ Dha17-3 } & $104.4 ; \mathrm{CH}_{2}$ & $\mathrm{H}_{\mathrm{A}}: 6.29(\mathrm{~d}, 1.4)$ & Dha17-1; Dha17-2 & Dha17-3- $\mathrm{H}_{\mathrm{B}}$ \\
\hline & & $\mathrm{H}_{\mathrm{B}}: 5.49(\mathrm{~d}, 1.3)$ & Dha17-1; Dha17-2 & Dha17-3- $\mathrm{H}_{\mathrm{A}}$ \\
\hline \multicolumn{5}{|l|}{$Q$} \\
\hline Q-1 & 162.7; C q & & & \\
\hline Q-2 & $152.7 ; \mathrm{C} \mathrm{q}$ & & & \\
\hline Q-3 & $117.4 ; \mathrm{CH}$ & $7.23(\mathrm{~s})$ & Q-1; Q-2; Q-10 & \\
\hline Q-4 & 151.7; C q & & & \\
\hline Q-5 & 127.4; $\mathrm{C} \mathrm{q}$ & & & \\
\hline Q-6 & 122.0; CH & $6.81(\mathrm{dd}, 10.1,2.3)$ & Q-5; Q-8; Q-10 & Q-7; Q-8 \\
\hline Q-7 & $135.0 \mathrm{CH}$ & $5.98(\mathrm{~m})$ & Q-5; Q-9 & Q-6; Q-8 \\
\hline Q-8 & $62.9 ; \mathrm{CH}$ & $3.50-3.40(\mathrm{~m})$ & Q-7; Q-9 & Q-6; Q-7; Q-9 \\
\hline Q-9 & $73.9 ; \mathrm{CH}$ & $4.52(\mathrm{~d}, 12.4)$ & Q-8; Q-10 & Q-8 \\
\hline Q-10 & 154.0; C q & & & \\
\hline Q-11 & $65.7 ; \mathrm{CH}$ & $4.95(\mathrm{q}, 6.5)$ & Q-4; Q-5; Q-6; Q-12 & Q-12 \\
\hline Q-12 & $23.8 ; \mathrm{CH}_{3}$ & $1.33(\mathrm{~d}, 6.6)$ & Q-4; Q-11 & Q-11 \\
\hline
\end{tabular}

${ }^{\text {a }} \mathrm{HMBC}$ correlations are from the proton to the indicated carbon.

${ }^{\mathrm{b}} \mathrm{COSY}$ correlations are from the proton to the proton attached to the indicated position. 
Figure S26. MS analysis of thiostrepton Ala2Met isolated from S. laurentii NDS1/int-A2M.

(A) HPLC-MS analysis. (1) Chromatogram extracted for $\mathrm{m} / \mathrm{z} 862.8$, the calculated $[\mathrm{M}+2 \mathrm{H}]^{2+}$ ion of thiostrepton Ala2Met. (2) Total ion chromatogram.

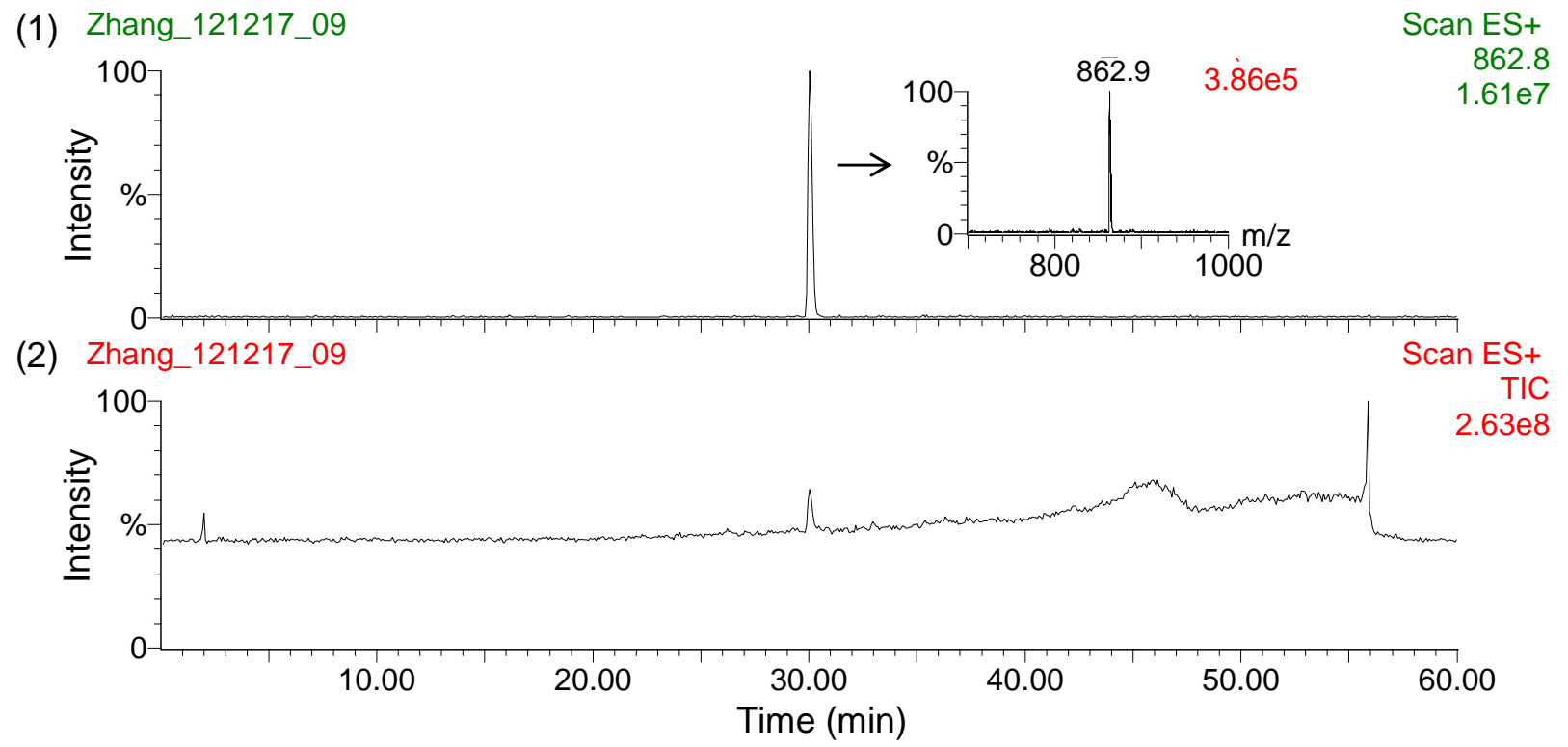

(B) MALDI MS spectrum of thiostrepton Ala2Met.

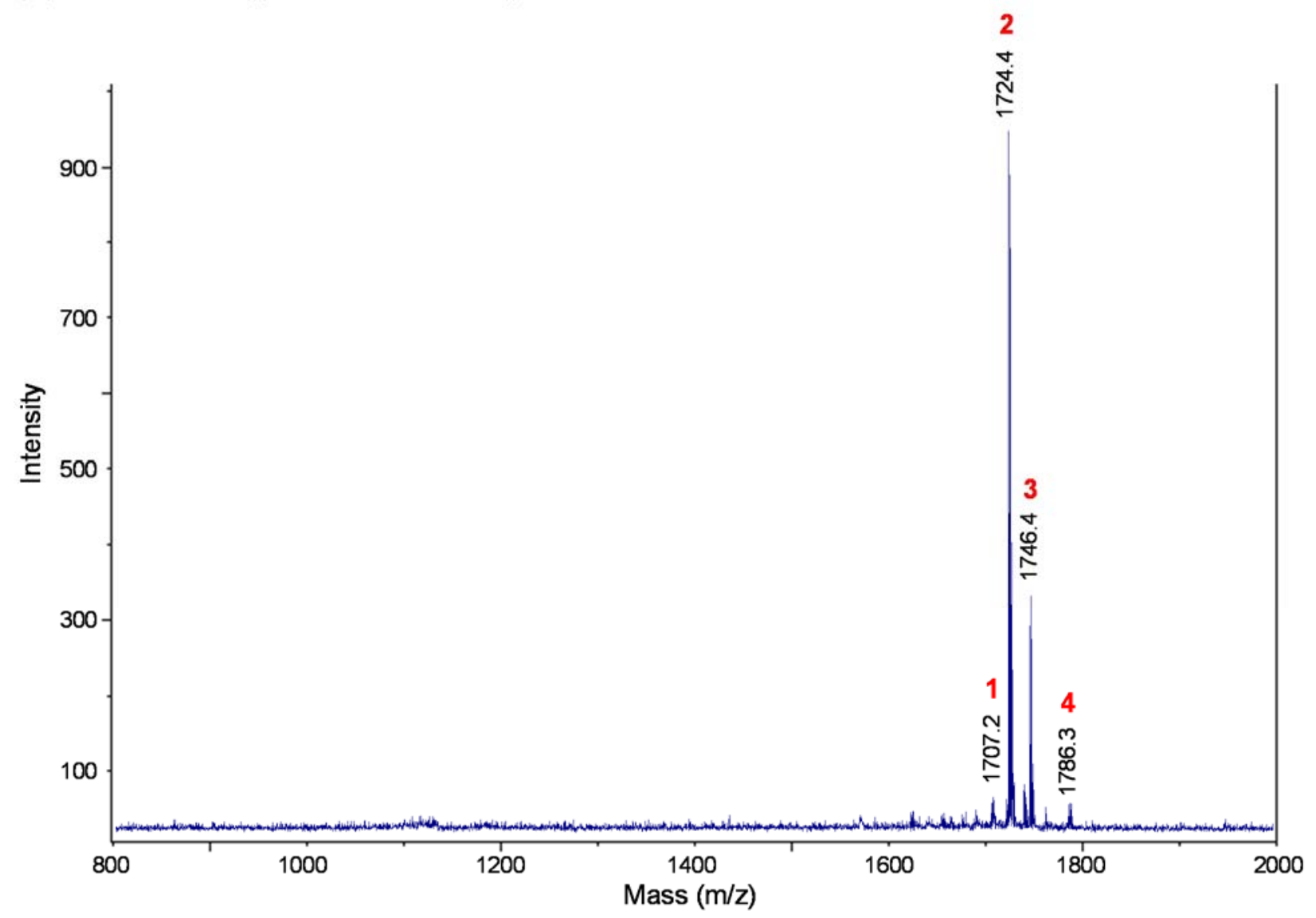


(C) MALDI MS/MS of parent ion $\mathrm{m} / \mathrm{z}$ 1724.4.

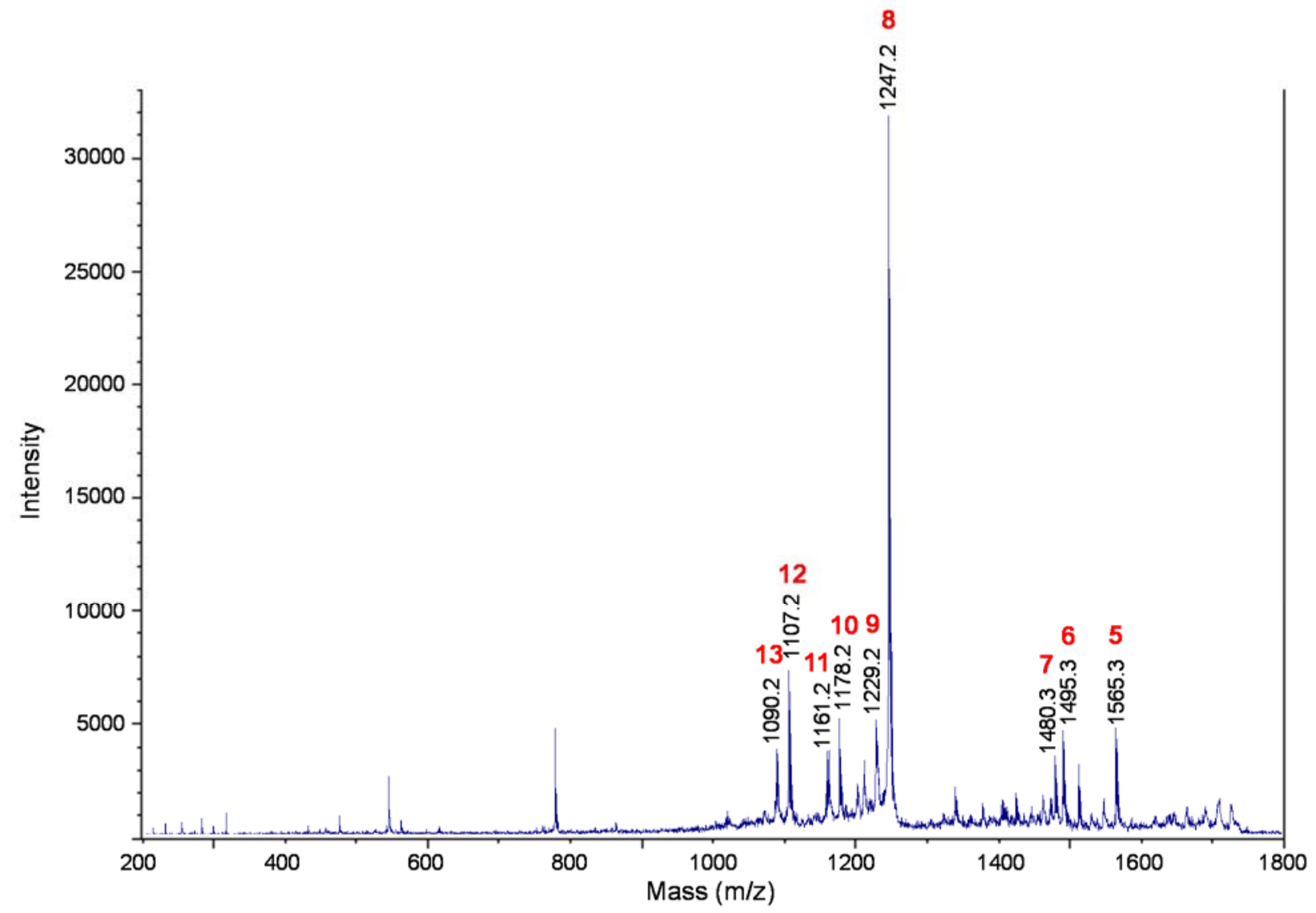

(D) Table and structure showing key ions and fragments in the MALDI MS and MS/MS of thiostrepton Ala2Met.

\begin{tabular}{|c|c|c|}
\hline Fragment & Expected & Observed \\
\hline 1. $\mathrm{M}-\mathrm{OH}+\mathrm{H}^{+}$ & 1707.5 & 1707.2 \\
\hline 2. $\mathrm{M}+\mathrm{H}^{+}$(Parent ion) & 1724.5 & 1724.4 \\
\hline 3. $\mathrm{M}+\mathrm{Na}^{+}$ & 1746.5 & 1746.4 \\
\hline 4. $\mathrm{M}+\mathrm{Cu}^{+}$ & 1786.4 & 1786.3 \\
\hline 5. M-Met2-CO+H ${ }^{+}$ & 1565.5 & 1565.3 \\
\hline 6. M-(Ile1-NH)-Met2+H+ & 1495.4 & 1495.3 \\
\hline 7. M-Ile1-Met2+H+ & 1480.4 & 1480.3 \\
\hline 8. M-QA-lle1-Met2+H ${ }^{+}$ & 1247.3 & 1247.2 \\
\hline 9. M-QA-lle1-Met2- $\mathrm{H}_{2} \mathrm{O}+\mathrm{H}^{+}$ & 1229.3 & 1229.2 \\
\hline 10. M-QA-lle1-Met2-Dha3+H+ ${ }^{+}$ & 1178.3 & 1178.2 \\
\hline 11. M-QA-lle1-Met2-Dha3-OH+H ${ }^{+}$ & 1161.3 & 1161.2 \\
\hline 12. M-QA-Ile1-Met2-Dha3-Ala4+H ${ }^{+}$ & 1107.3 & 1107.2 \\
\hline 13. M-QA-Ile1-Met2-Dha3-Ala4-OH+H+ & 1090.2 & 1090.2 \\
\hline
\end{tabular}

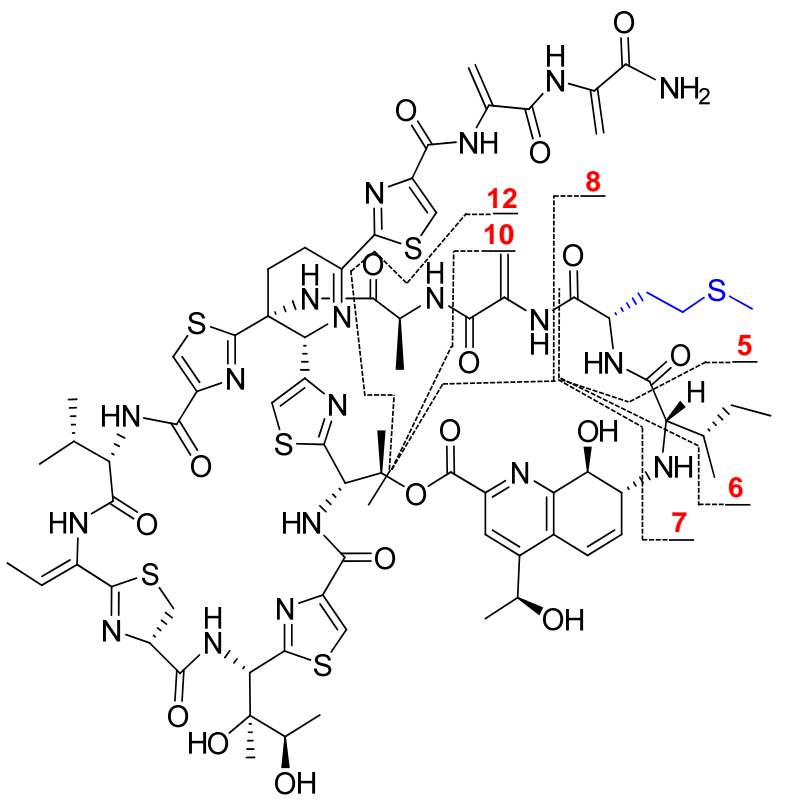


Figure S27. MS analysis of thiostrepton Ala2Phe isolated from S. laurentii NDS1/int-A2F.

(A) HPLC-MS analysis. (1) Chromatogram extracted for $\mathrm{m} / \mathrm{z} 870.8$, the calculated $[\mathrm{M}+2 \mathrm{H}]^{2+}$ ion of thiostrepton Ala2Phe. (2) Total ion chromatogram.

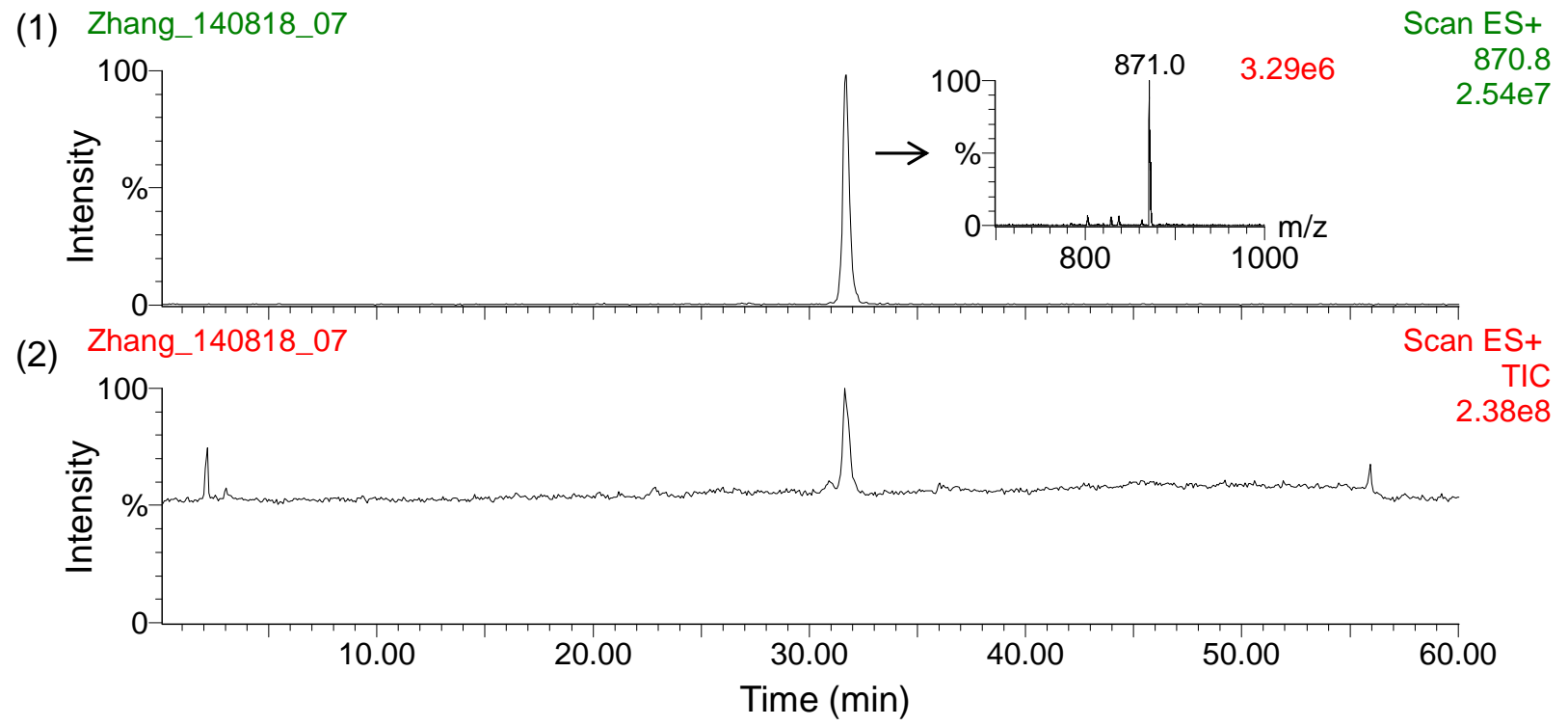

(B) MALDI MS spectrum of thiostrepton Ala2Phe.

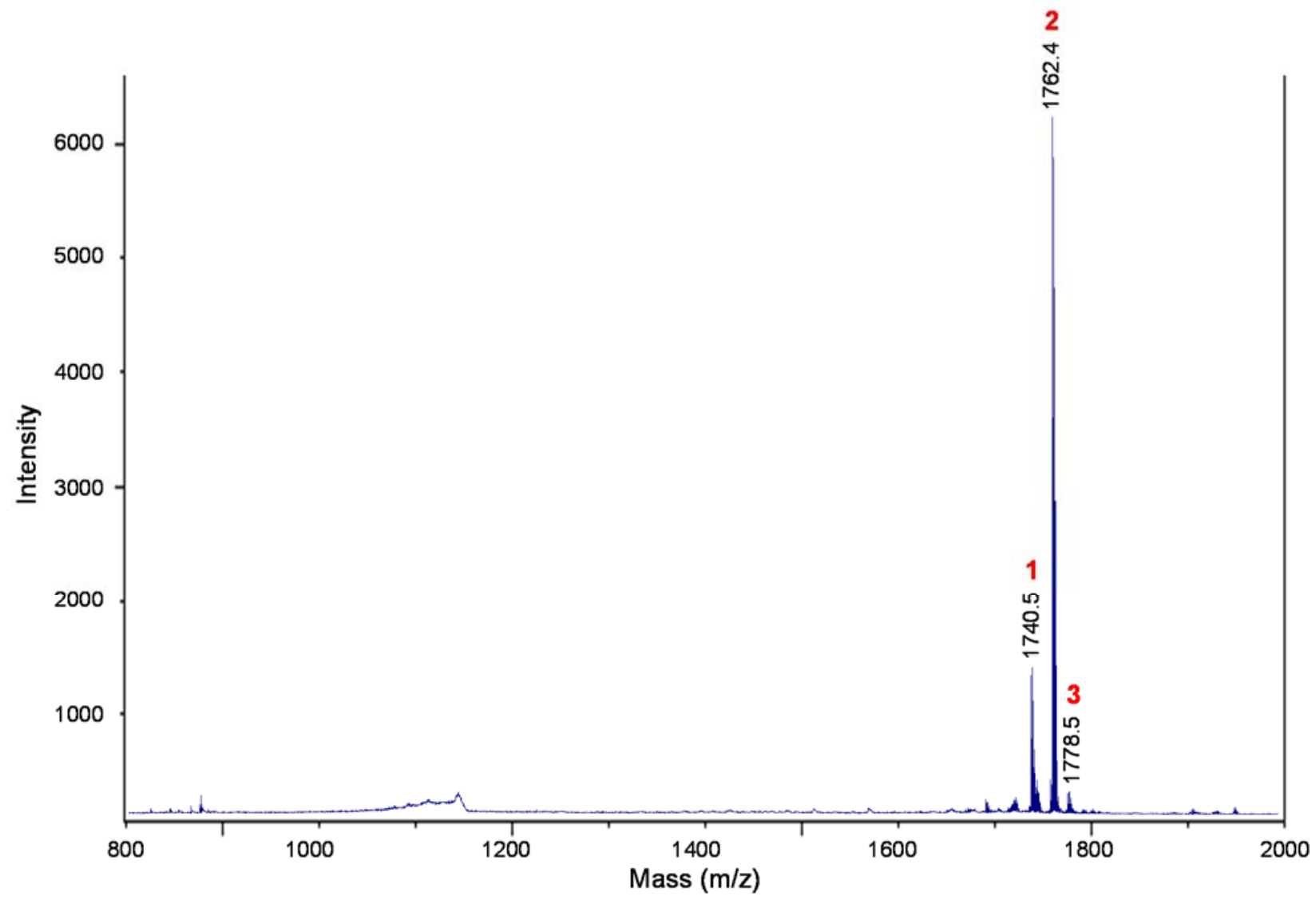


(C) MALDI MS/MS of parent ion $\mathrm{m} / \mathrm{z} 1740.5$.

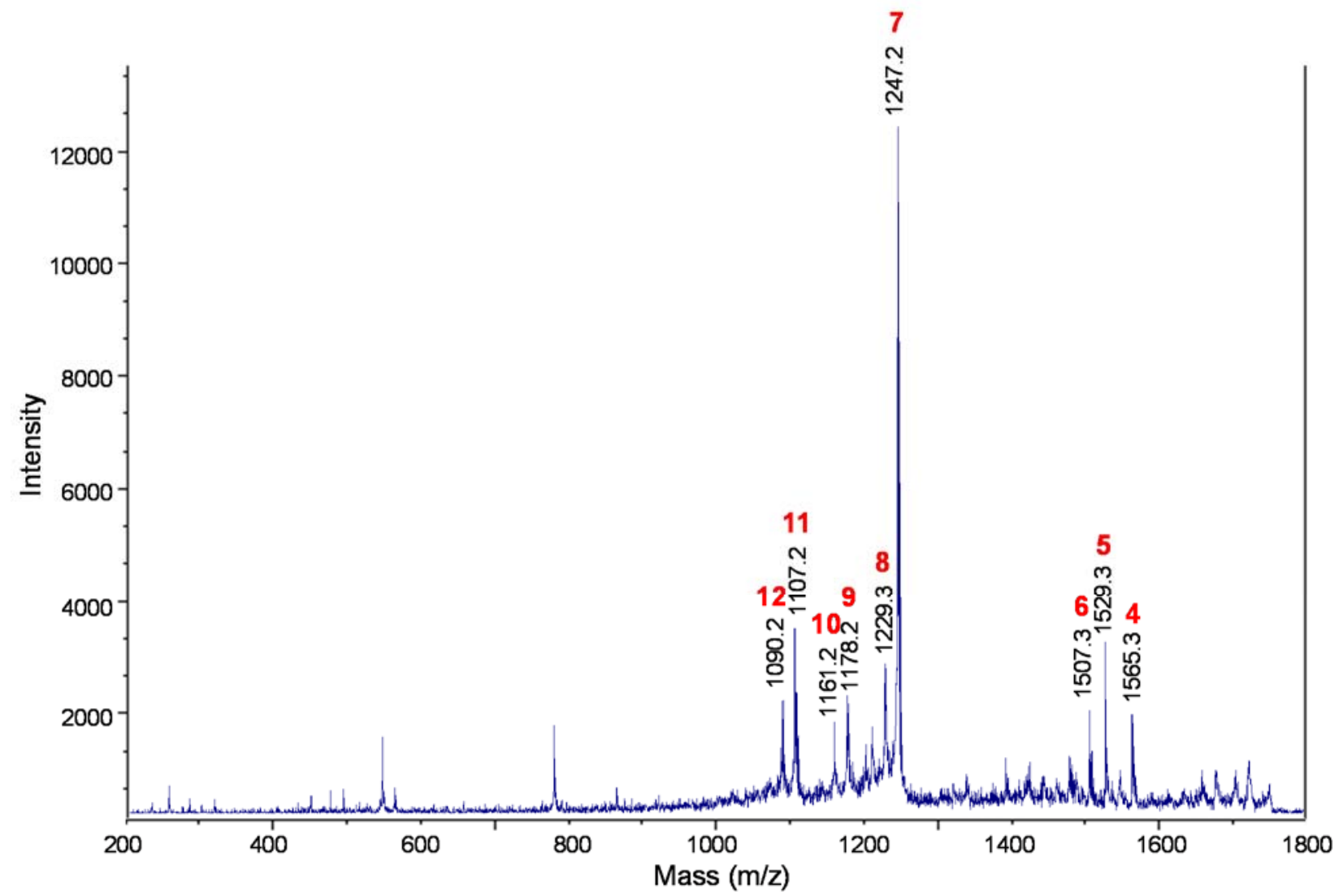

(D) Table and structure showing key ions and fragments in the MALDI MS and MS/MS of thiostrepton Ala2Phe.

\begin{tabular}{|c|c|c|}
\hline Fragment & Expected & Observed \\
\hline 1. $\mathrm{M}+\mathrm{H}^{+}$(Parent ion) & 1740.5 & 1740.5 \\
\hline 2. $\mathrm{M}+\mathrm{Na}^{+}$ & 1762.5 & 1762.4 \\
\hline 3. $\mathrm{M}+\mathrm{K}^{+}$ & 1778.5 & 1778.5 \\
\hline 4. $\mathrm{M}-\mathrm{Phe} 2-\mathrm{CO}+\mathrm{H}^{+}$ & 1565.5 & 1565.3 \\
\hline 5. M-Dhb8-Tzn9-CO-NH+H & 1529.5 & 1529.3 \\
\hline 6. $M-Q A+H^{+}$ & 1507.5 & 1507.3 \\
\hline 7. M-QA-lle1-Phe2+H+ & 1247.3 & 1247.2 \\
\hline 8. M-QA-lle1-Phe2- $\mathrm{H}_{2} \mathrm{O}+\mathrm{H}^{+}$ & 1229.3 & 1229.3 \\
\hline 9. M-QA-lle1-Phe2-Dha3+H ${ }^{+}$ & 1178.3 & 1178.2 \\
\hline 10. M-QA-lle1-Phe2-Dha3-OH+H+ & 1161.3 & 1161.2 \\
\hline 11. M-QA-Ile1-Phe2-Dha3-Ala4+H+ & 1107.3 & 1107.2 \\
\hline 12. M-QA-Ile1-Phe2-Dha3-Ala4-OH+H+ & 1090.2 & 1090.2 \\
\hline
\end{tabular}

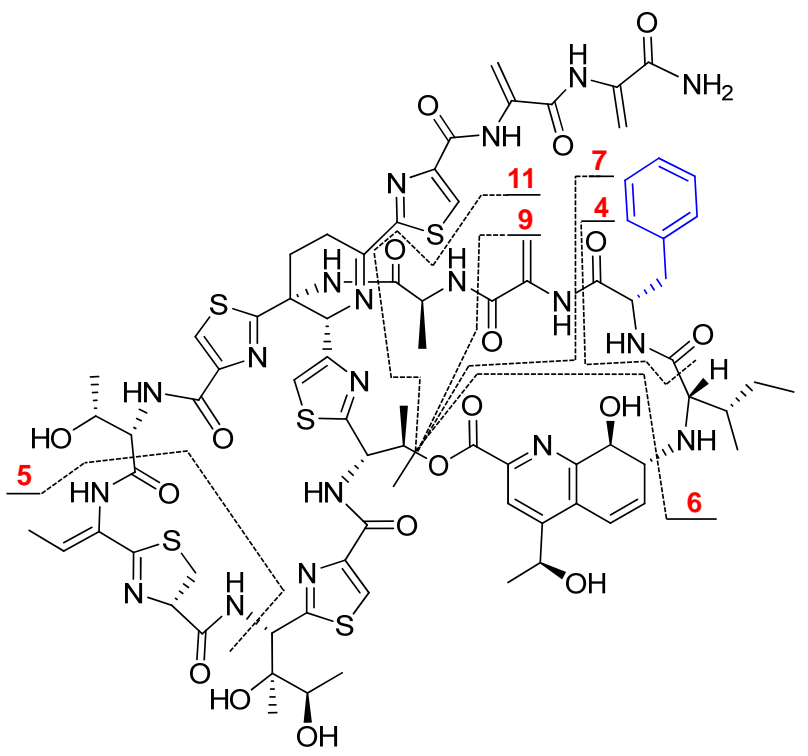


Figure S28. MS analysis of thiostrepton Ala2Tyr isolated from S. laurentii NDS1/int-A2Y.

(A) HPLC-MS analysis. (1) Chromatogram extracted for $\mathrm{m} / \mathrm{z} 878.8$, the calculated $[\mathrm{M}+2 \mathrm{H}]^{2+}$ ion of thiostrepton Ala2Tyr. (2) Total ion chromatogram.

(1) Zhang_140818_10

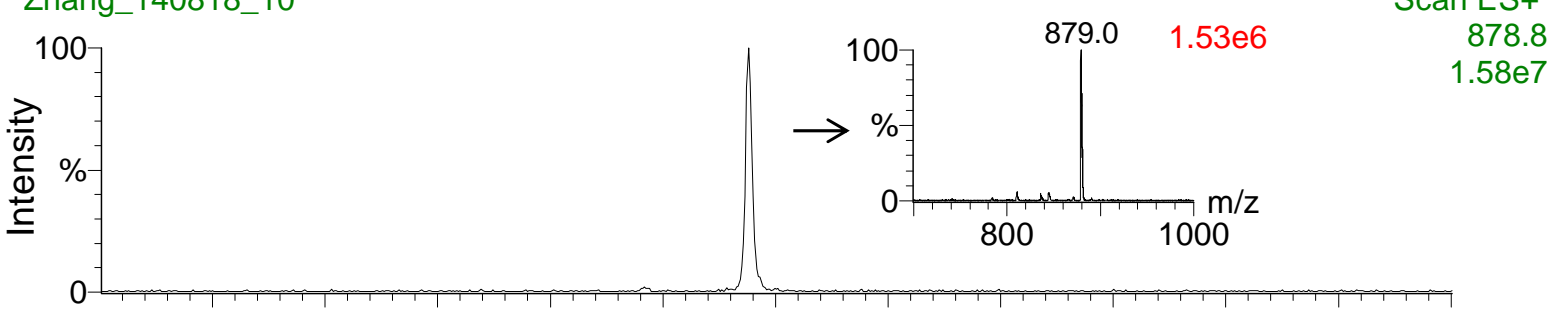

(2)

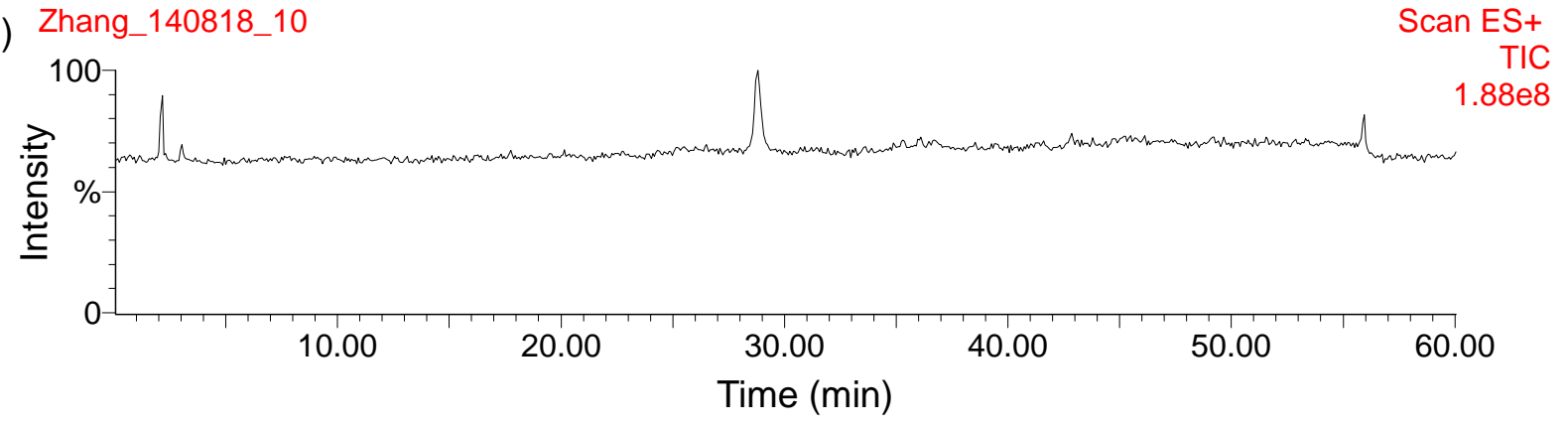

(B) MALDI MS spectrum of thiostrepton Ala2Tyr.

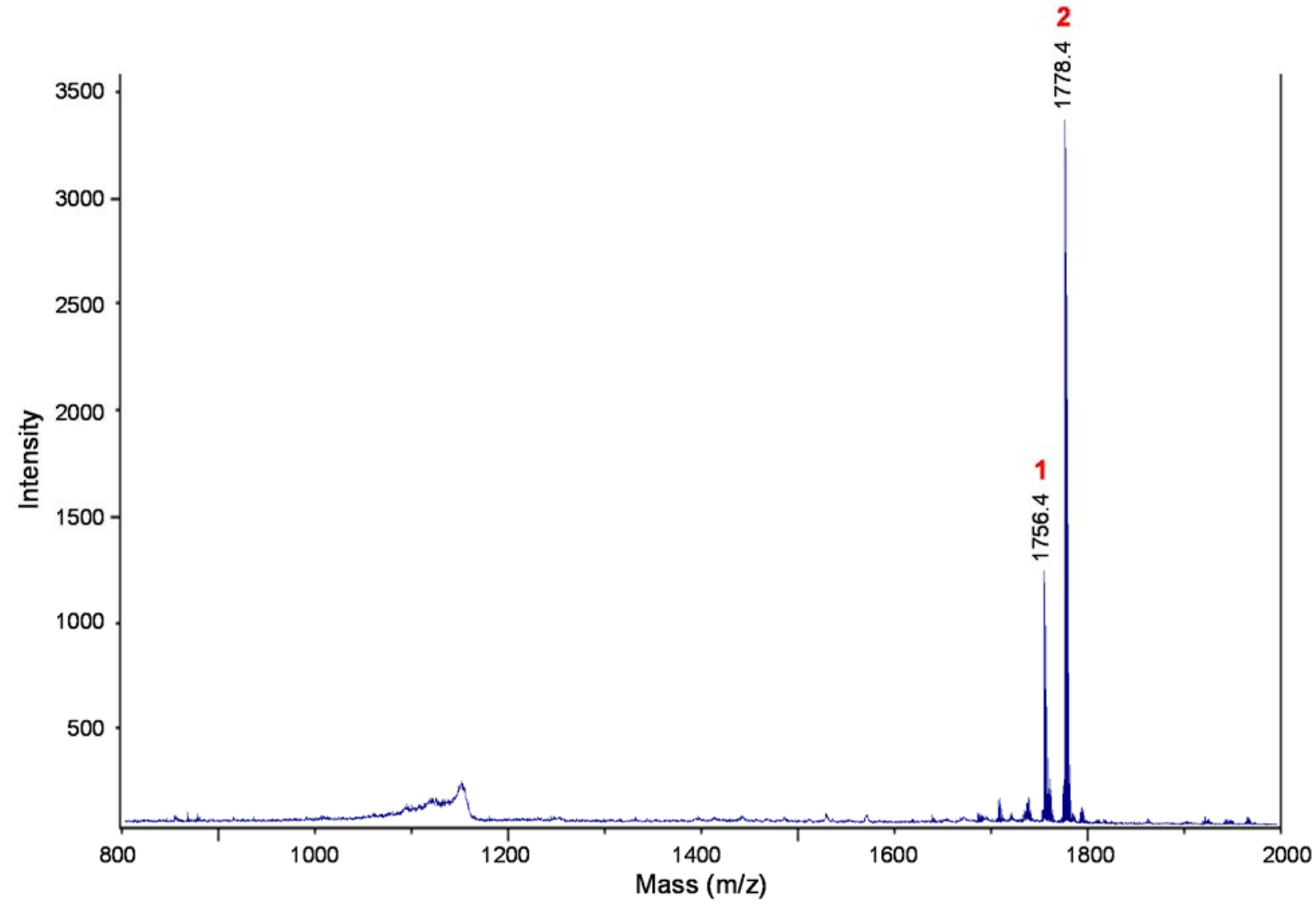


(C) MALDI MS/MS of parent ion $m / z$ 1756.4.

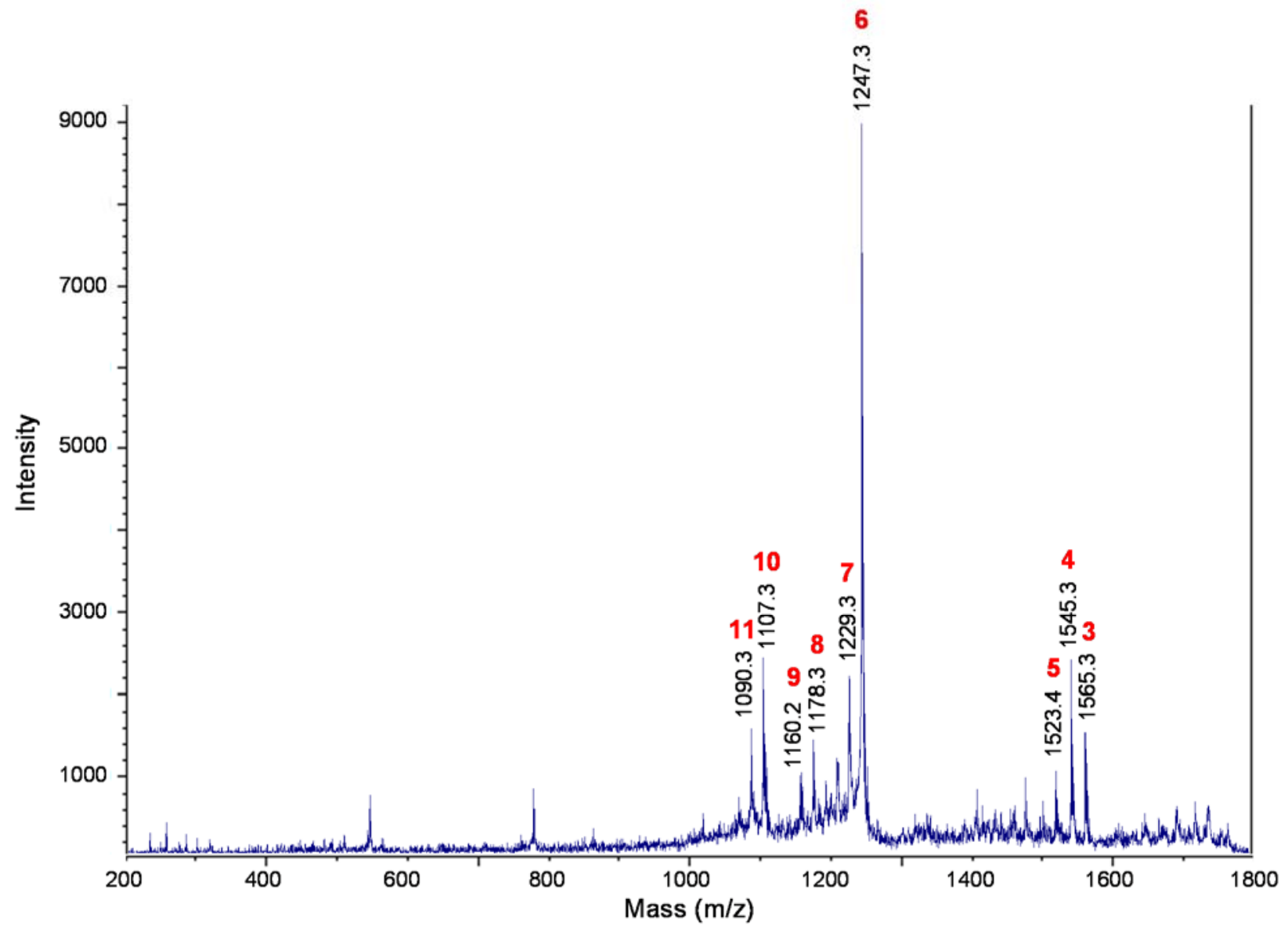

(D) Table and structure showing key ions and fragments in the MALDI MS and MS/MS of thiostrepton Ala2Tyr.

\begin{tabular}{|c|c|c|}
\hline Fragment & Expected & Observed \\
\hline 1. $\mathrm{M}+\mathrm{H}^{+}$(Parent ion) & 1756.5 & 1756.4 \\
\hline 2. $\mathrm{M}+\mathrm{Na}^{+}$ & 1778.5 & 1778.4 \\
\hline 3. $\mathrm{M}-\mathrm{Tyr} 2-\mathrm{CO}+\mathrm{H}^{+}$ & 1565.5 & 1565.3 \\
\hline 4. M-Dhb8-Tzn9-CO-NH+H+ & 1545.5 & 1545.3 \\
\hline 5. $\mathrm{M}-\mathrm{QA}+\mathrm{H}^{+}$ & 1523.5 & 1523.4 \\
\hline 6. M-QA-Ile1-Tyr2+H+ & 1247.3 & 1247.3 \\
\hline 7. M-QA-Ile1-Tyr2- $\mathrm{H}_{2} \mathrm{O}+\mathrm{H}^{+}$ & 1229.3 & 1229.3 \\
\hline 8. M-QA-Ile1-Tyr2-Dha3+H+ & 1178.3 & 1178.3 \\
\hline 9. M-QA-lle1-Tyr2-Dha3- $\mathrm{H}_{2} \mathrm{O}+\mathrm{H}^{+}$ & 1160.3 & 1160.2 \\
\hline 10. M-QA-lle1-Tyr2-Dha3-Ala4+H' & 1107.3 & 1107.3 \\
\hline 11. M-QA-Ile1-Tyr2-Dha3-Ala4-OH+H+ & 1090.2 & 1090.3 \\
\hline
\end{tabular}

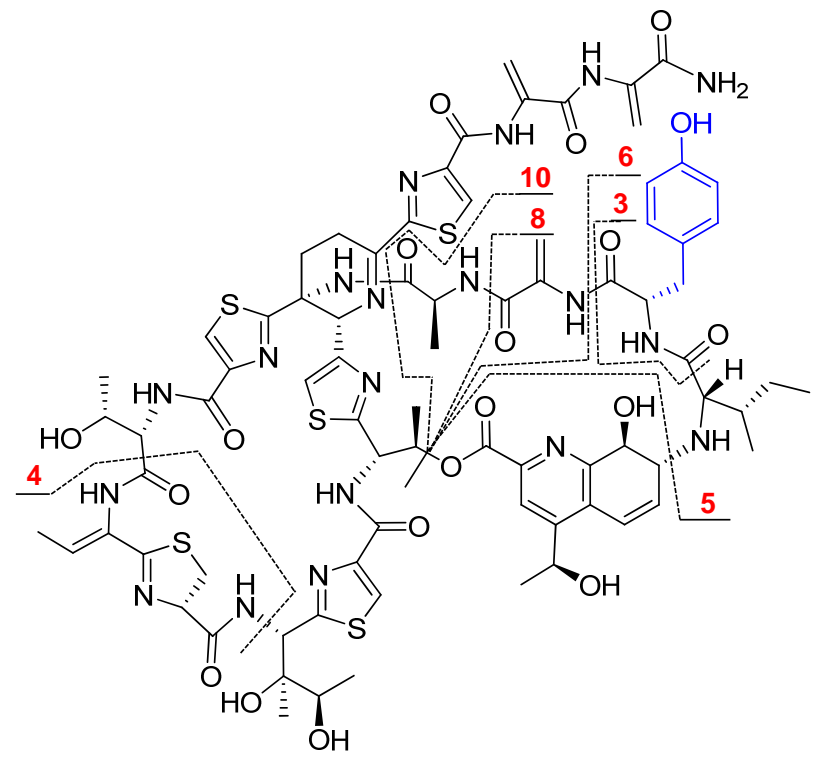


Figure S29. MS analysis of thiostrepton Ala2Val- $\Delta$ Ile1 isolated from S. laurentii NDS1/intA2V.

(A) HPLC-MS analysis. (1) Chromatogram extracted for $\mathrm{m} / \mathrm{z} 790.2$, the calculated $[\mathrm{M}+2 \mathrm{H}]^{2+}$ ion of thiostrepton Ala2Val- $\Delta$ Ile1. (2) Total ion chromatogram.

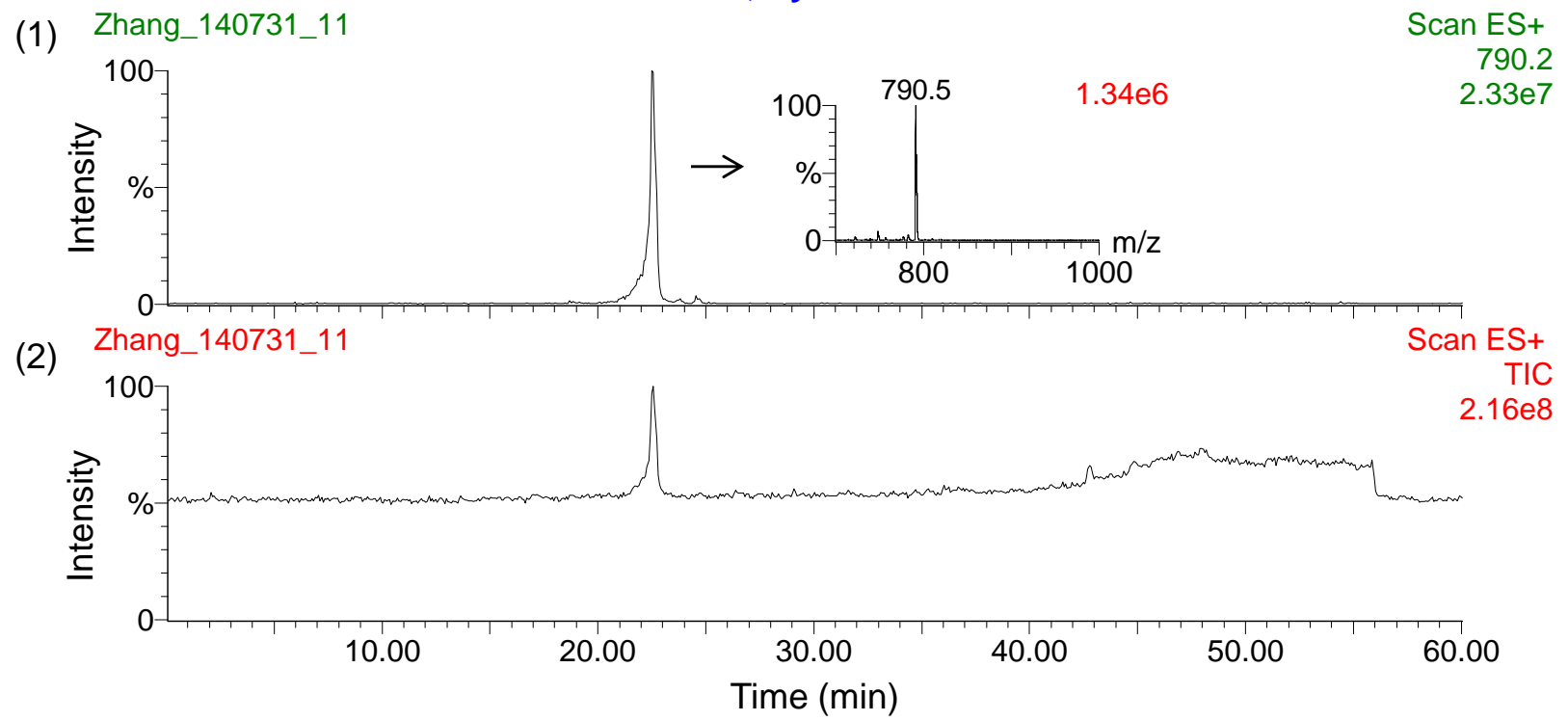

(B) MALDI MS spectrum of thiostrepton Ala2Val- $\Delta$ Ile1.

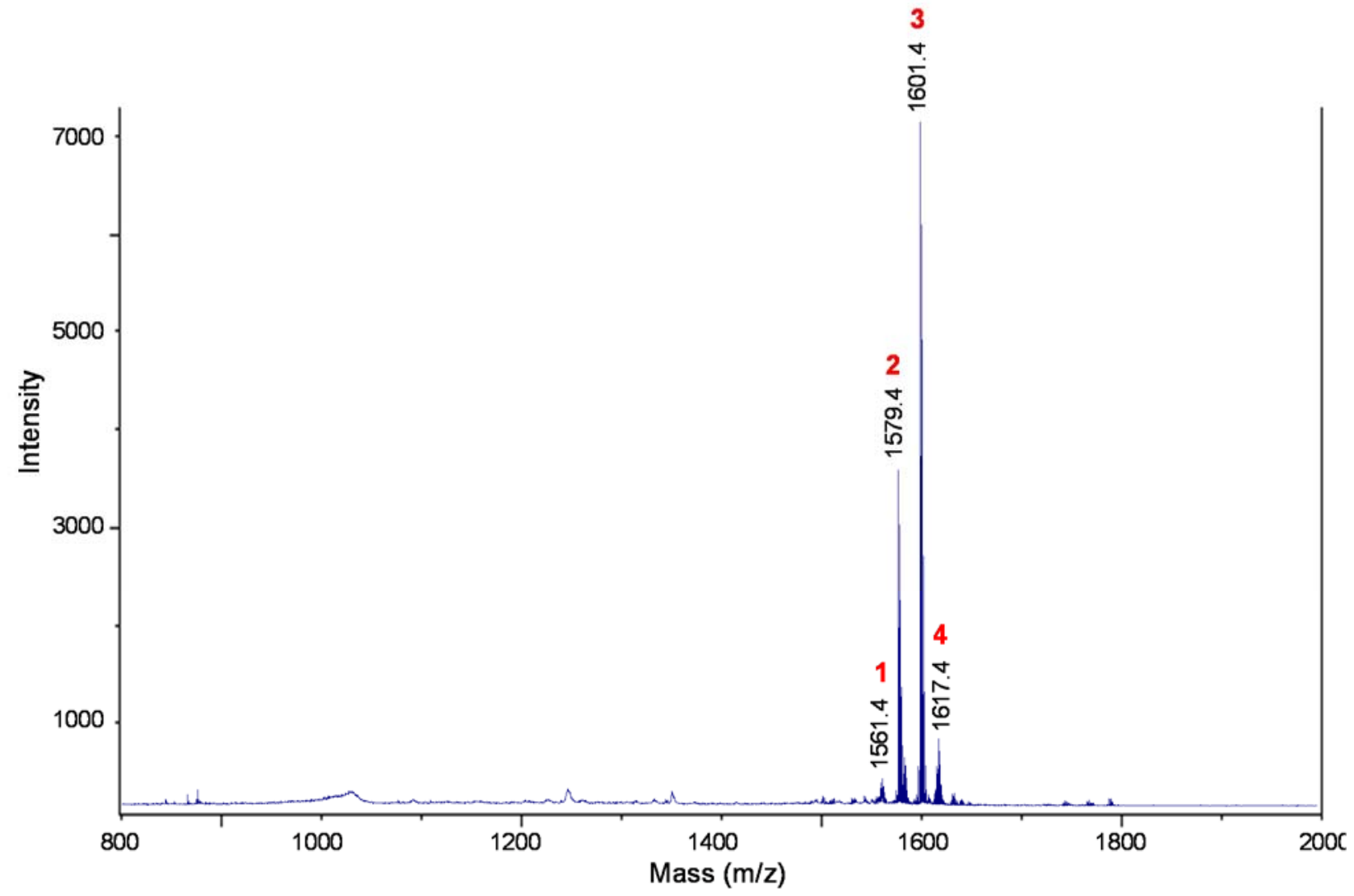


(C) MALDI MS/MS of parent ion $m / z$ 1579.4.

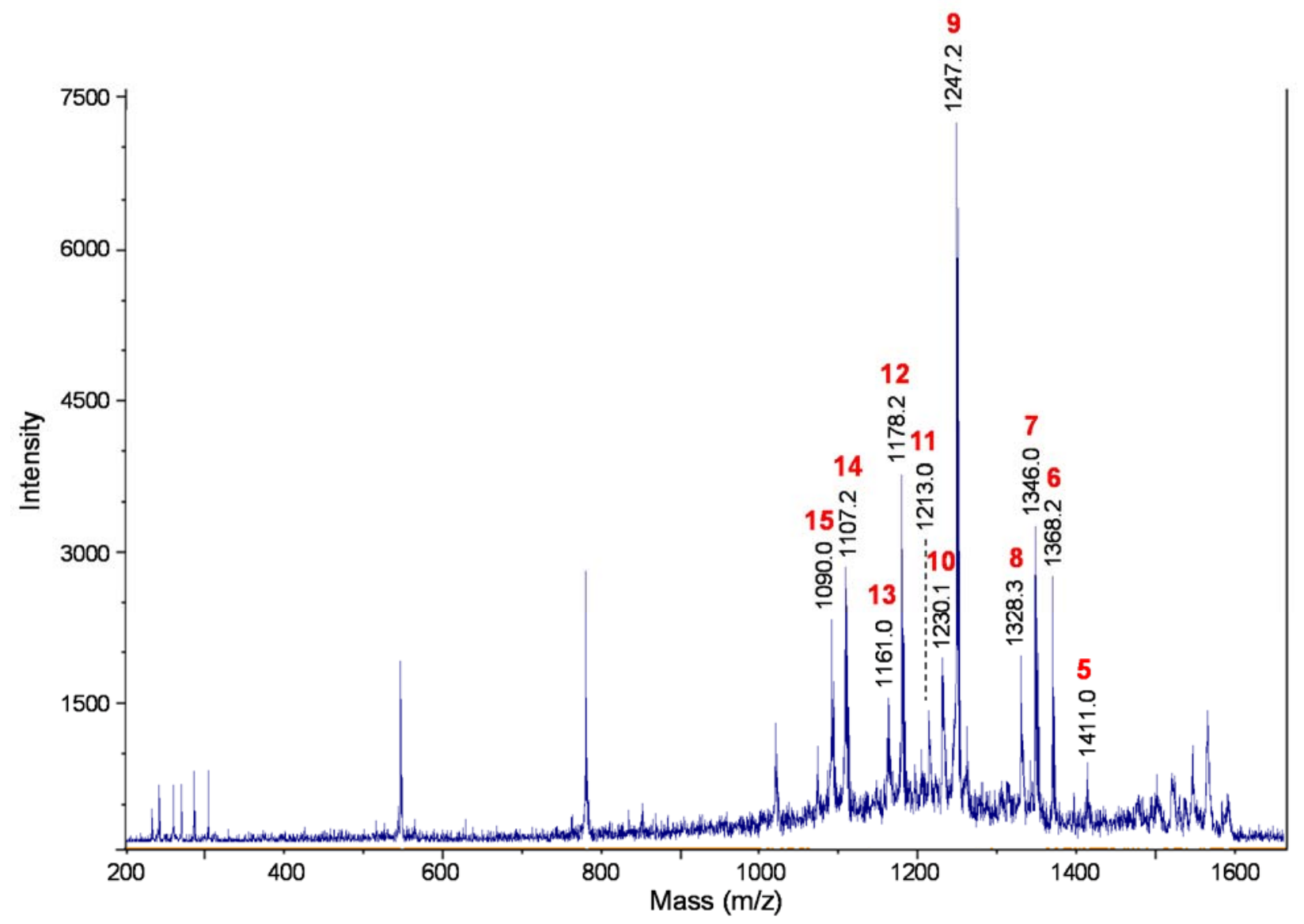

(D) Table and structure showing key ions and fragments in the MALDI MS and MS/MS of thiostrepton Ala2Val- $\Delta$ Ile1.

\begin{tabular}{|c|c|c|}
\hline Fragment & Expected & Observed \\
\hline 1. $\mathrm{M}-\mathrm{H}_{2} \mathrm{O}+\mathrm{H}^{+}$ & 1561.4 & 1561.4 \\
\hline 2. $\mathrm{M}+\mathrm{H}^{+}$(Parent ion) & 1579.4 & 1579.4 \\
\hline 3. $\mathrm{M}+\mathrm{Na}^{+}$ & 1601.4 & 1601.4 \\
\hline 4. $\mathrm{M}+\mathrm{K}^{+}$ & 1617.4 & 1617.4 \\
\hline 5. M-Dhb8-Tzn9+H ${ }^{+}$ & 1411.4 & 1411.0 \\
\hline 6. M-Val2-Dha3-(Ala4-CO)+H+ & 1368.3 & 1368.2 \\
\hline 7. $M-Q A+H^{+}$ & 1346.4 & 1346.3 \\
\hline 8. $\mathrm{M}-\mathrm{QA}-\mathrm{H}_{2} \mathrm{O}+\mathrm{H}^{+}$ & 1328.4 & 1328.3 \\
\hline 9. $\mathrm{M}-\mathrm{QA}-\mathrm{Val} 2+\mathrm{H}^{+}$ & 1247.3 & 1247.2 \\
\hline 10. $\mathrm{M}-\mathrm{QA}-\mathrm{Val} 2-\mathrm{OH}+\mathrm{H}^{+}$ & 1230.3 & 1230.1 \\
\hline 11. M-QA-Val2-OH-OH+H ${ }^{+}$ & 1213.3 & 1213.0 \\
\hline 12. M-QA-Val2-Dha3+H+ & 1178.3 & 1178.2 \\
\hline 13. M-QA-Val2-Dha3-OH+H+ & 1161.3 & 1161.0 \\
\hline 14. M-QA-Val2-Dha3-Ala4+H ${ }^{+}$ & 1107.3 & 1107.2 \\
\hline 15. M-QA-Val2-Dha3-Ala4-OH+H+ & 1090.2 & 1090.0 \\
\hline
\end{tabular}

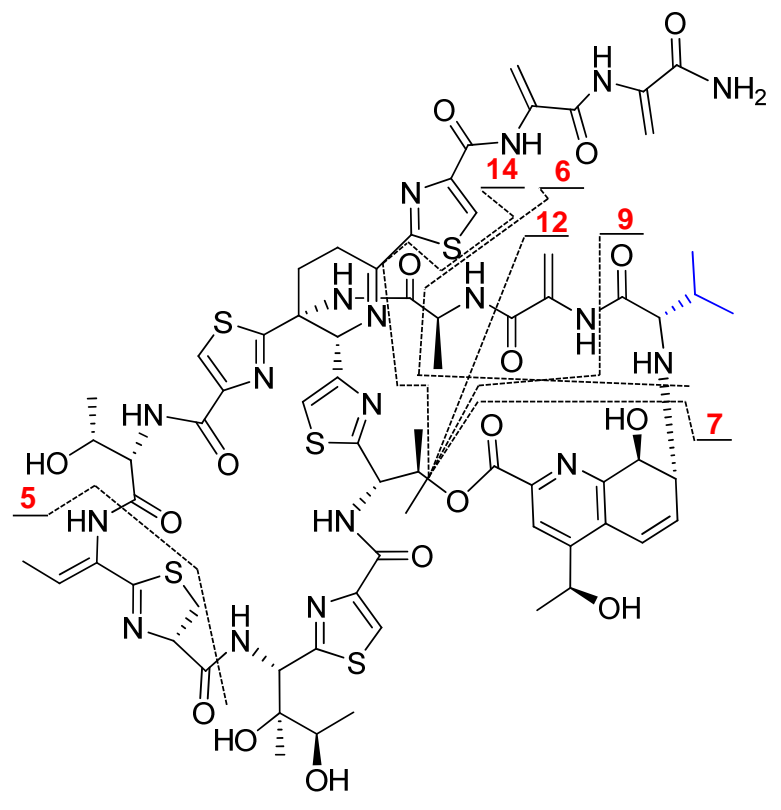


Figure S30. Structure and numbering system used for thiostrepton Ala2Val- $\Delta$ Ile1.

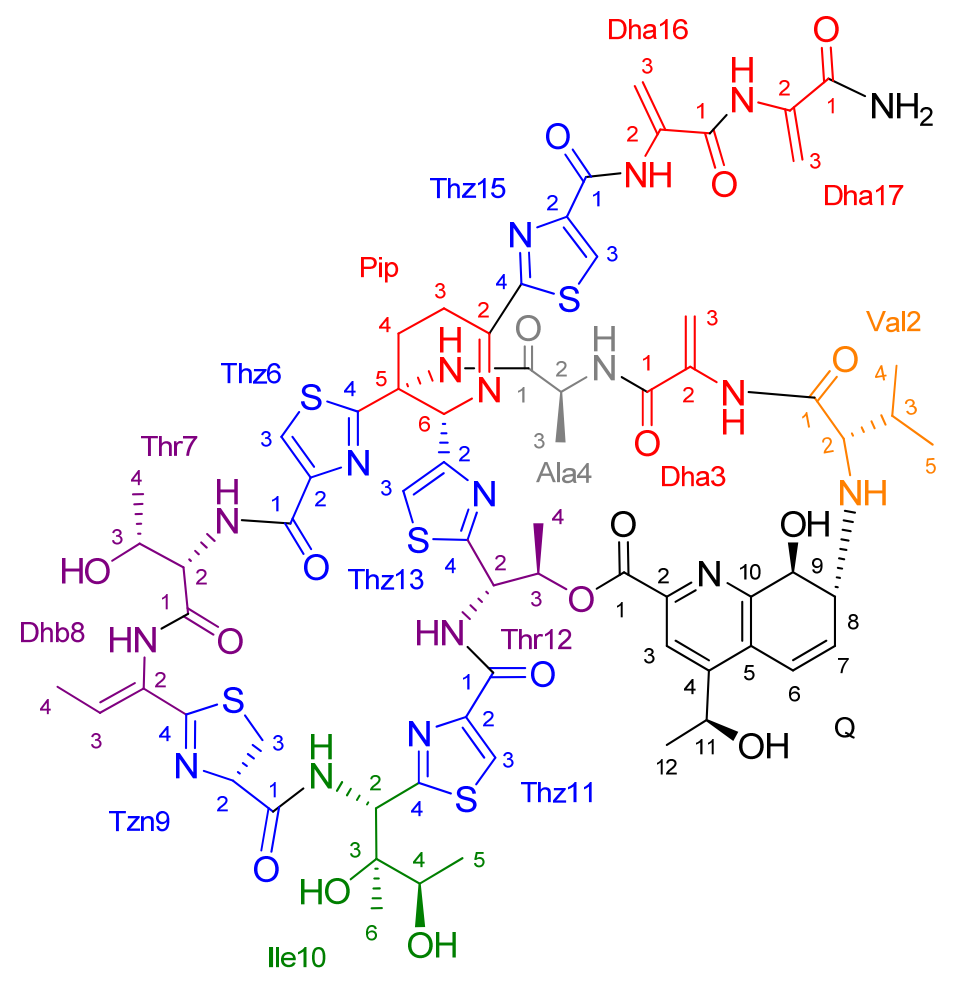




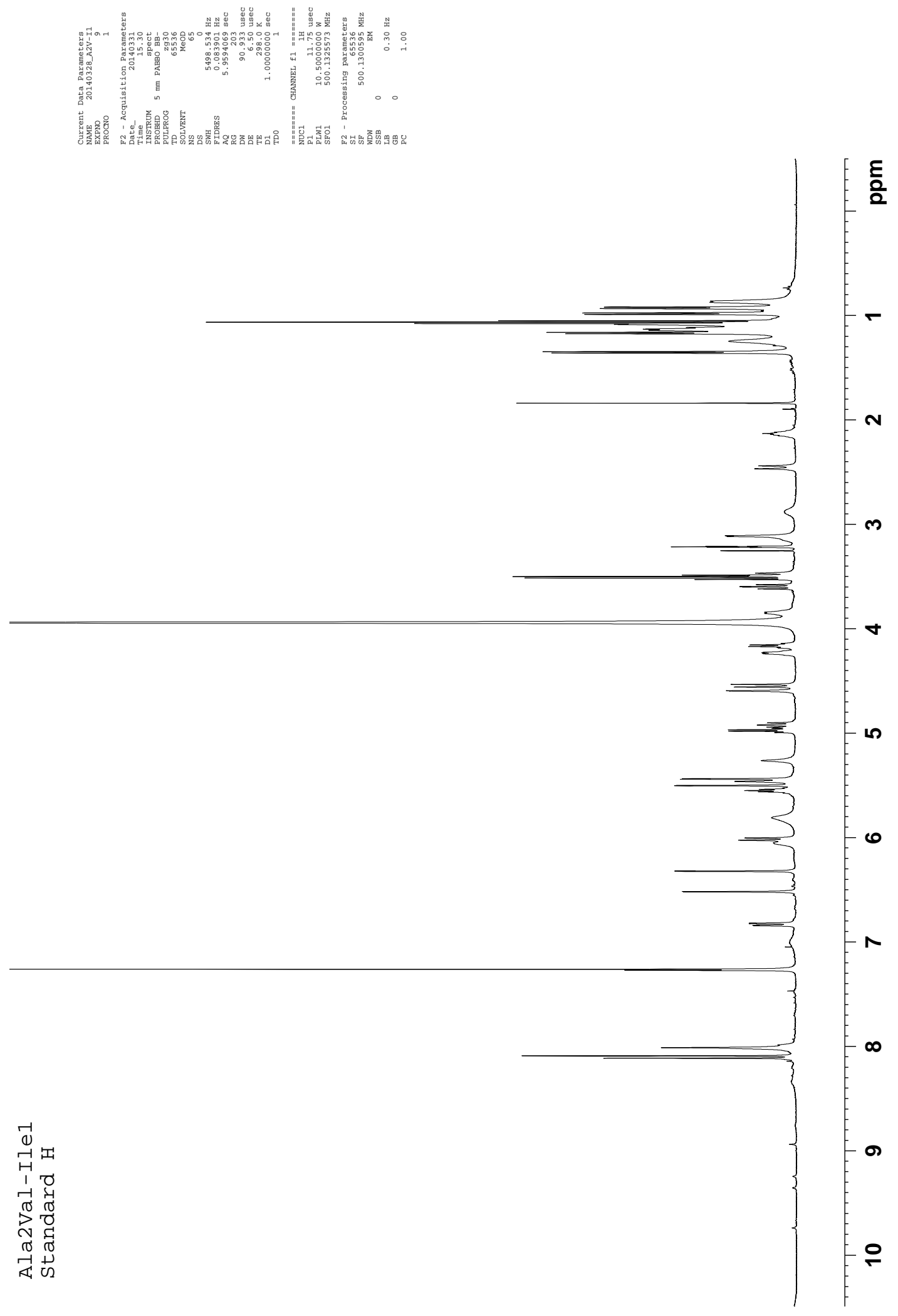

Figure S31. ${ }^{1} \mathrm{H}$ NMR spectrum of thiostrepton Ala2Val- $\Delta \mathrm{Ile} 1\left(500 \mathrm{MHz}, \mathrm{CDCl}_{3}-\mathrm{CD}_{3} \mathrm{OD}\right.$ 4:1, $25^{\circ} \mathrm{C}$ ). 

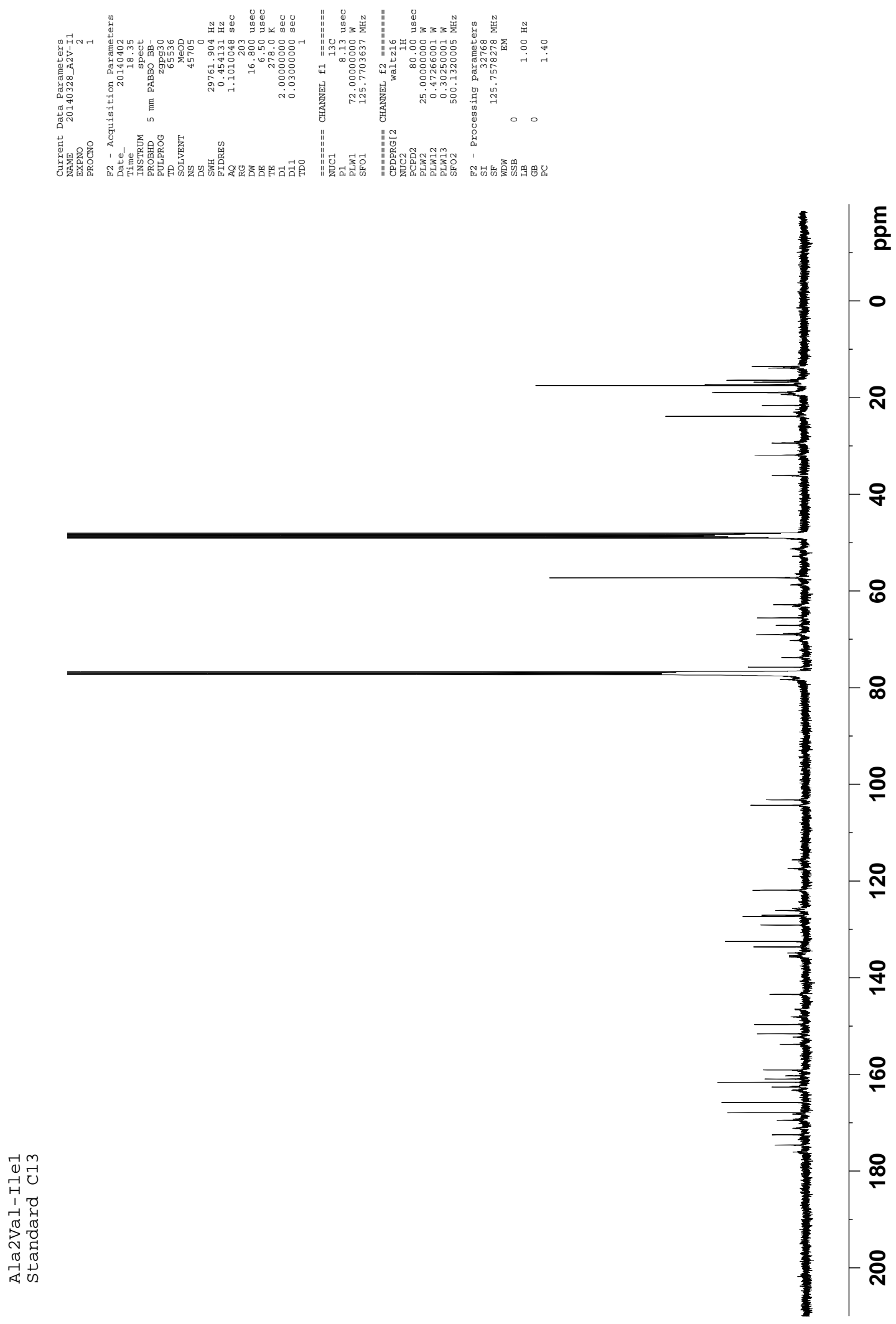

Figure S32. ${ }^{13} \mathrm{C}$ NMR spectrum of thiostrepton Ala2Val- $\Delta \mathrm{Ile} 1\left(125 \mathrm{MHz}, \mathrm{CDCl}_{3}-\mathrm{CD}_{3} \mathrm{OD}\right.$ 4:1, $25^{\circ} \mathrm{C}$ ). 


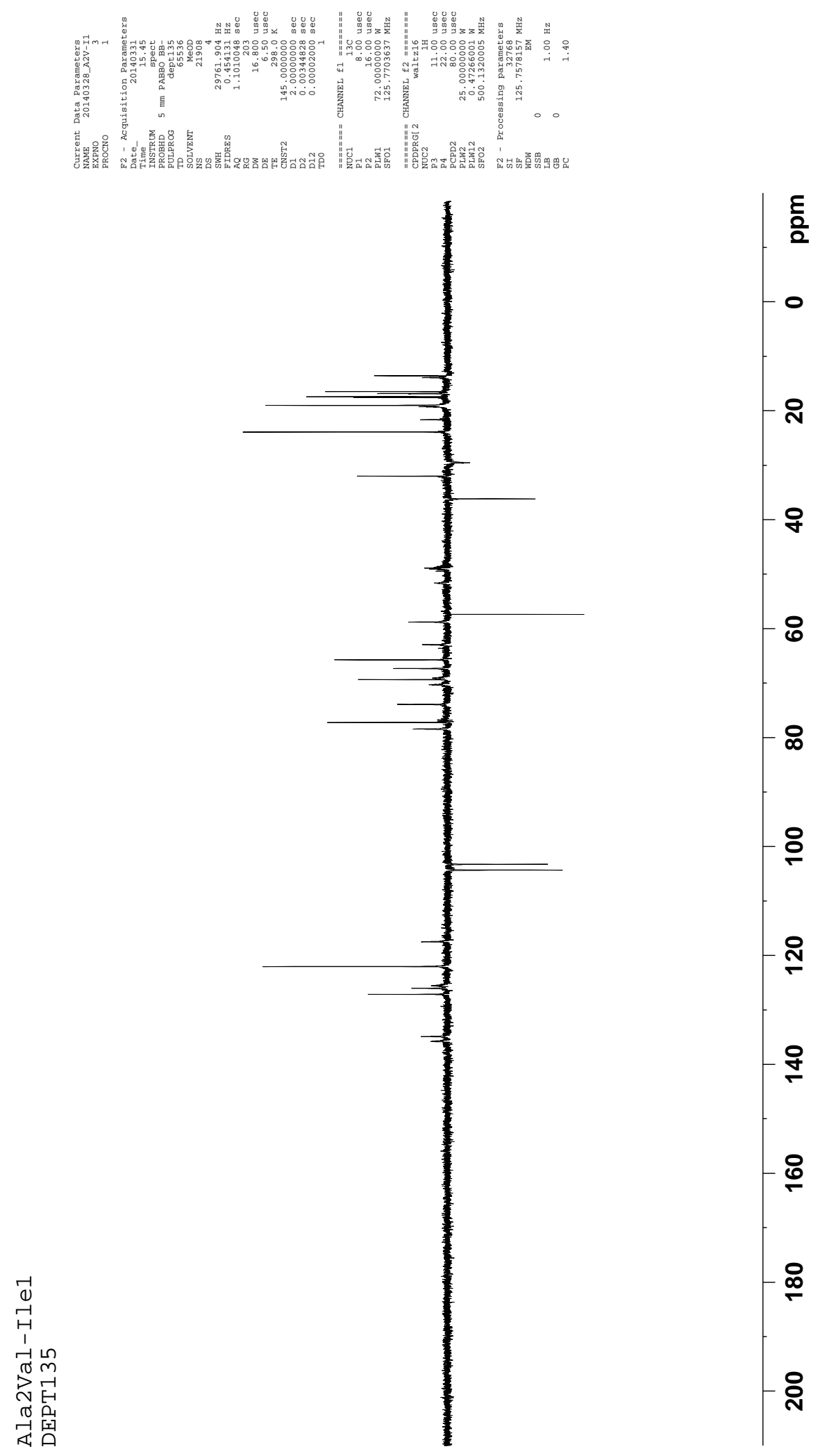

Figure S33. DEPT-135 NMR spectrum of thiostrepton Ala2Val- $\Delta$ Ile1 $\left(125 \mathrm{MHz}, \mathrm{CDCl}_{3}-\mathrm{CD}_{3} \mathrm{OD}\right.$ 4:1, $25^{\circ} \mathrm{C}$ ). 


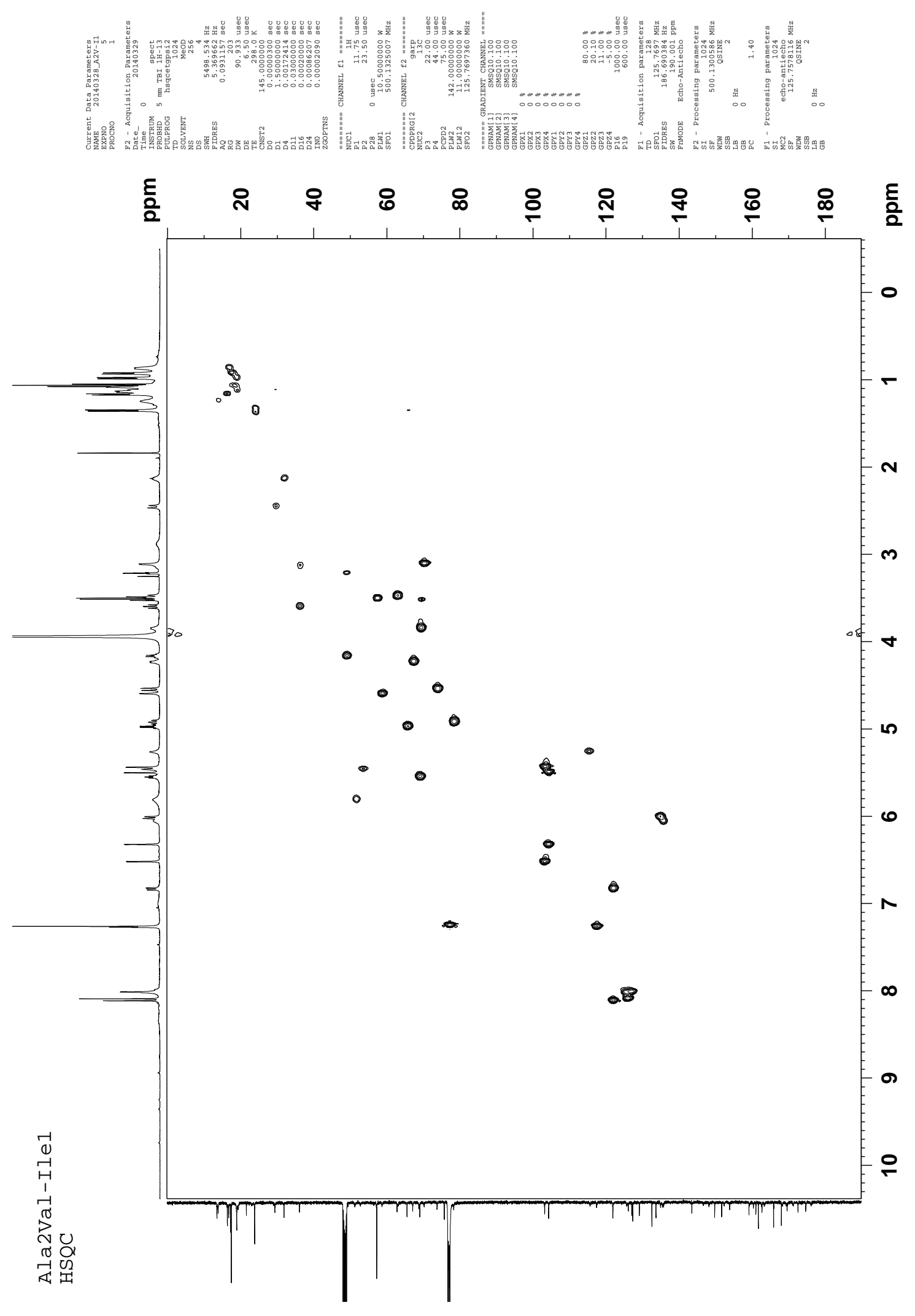

Figure S34. gHSQC NMR spectrum of thiostrepton Ala2Val- $\Delta$ Ile1 $\left(500 \mathrm{MHz}, \mathrm{CDCl}_{3}-\mathrm{CD}_{3} \mathrm{OD}\right.$ 4:1, $25^{\circ} \mathrm{C}$ ). 


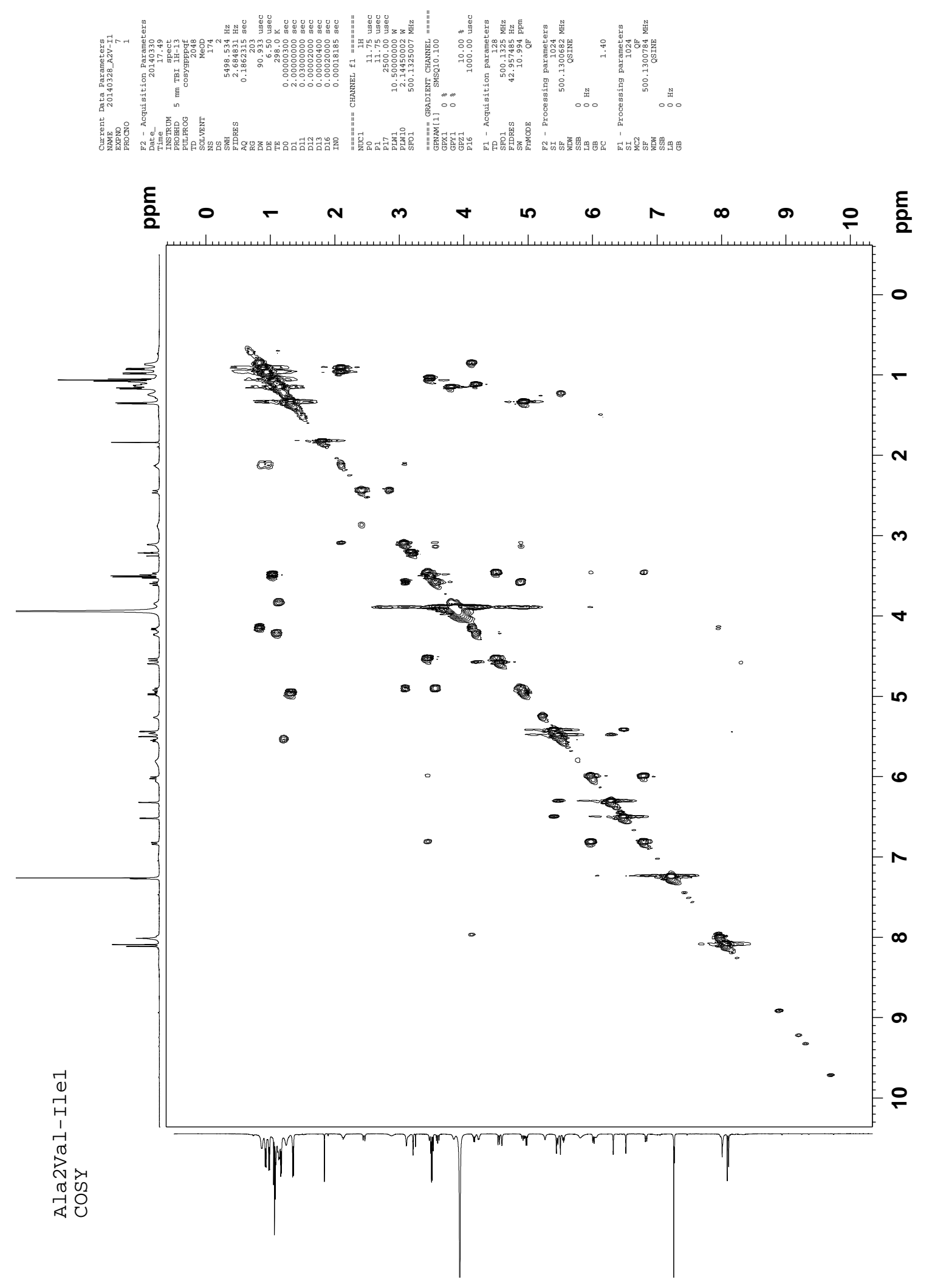

Figure S35. gCOSY NMR spectrum of thiostrepton Ala2Val- $\Delta$ Ile1 $\left(125 \mathrm{MHz}, \mathrm{CDCl}_{3}-\mathrm{CD}_{3} \mathrm{OD}\right.$ $\left.4: 1,25^{\circ} \mathrm{C}\right)$. 


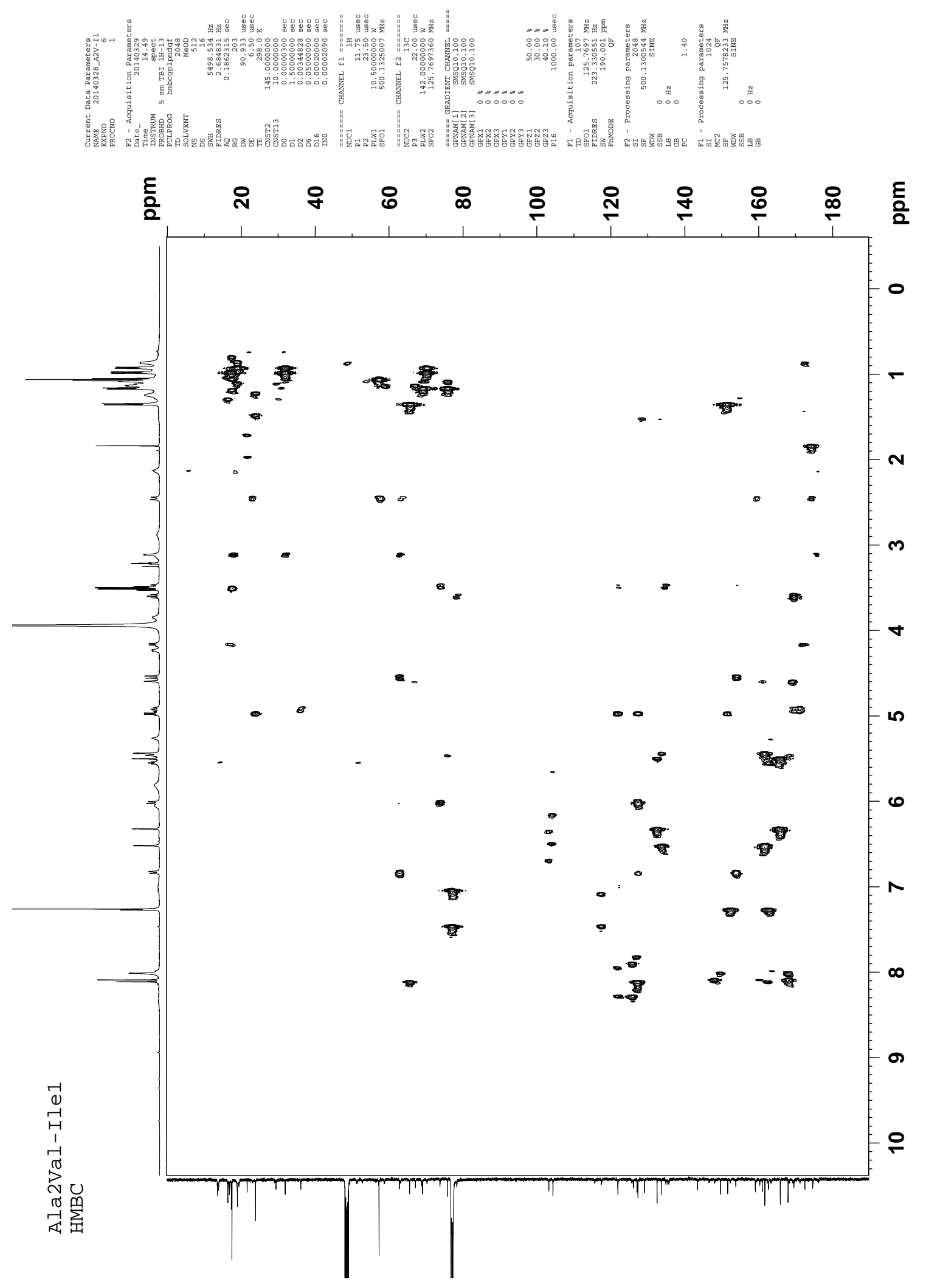

Figure S36. gHMBC NMR spectrum of thiostrepton Ala2Val- $\Delta$ Ile1 (125 MHz, $\mathrm{CDCl}_{3}-\mathrm{CD}_{3} \mathrm{OD}$ $\left.4: 1,25^{\circ} \mathrm{C}\right)$. 
Table S4. ${ }^{1} \mathrm{H}$ and ${ }^{13} \mathrm{C}$ NMR assignments of thiostrepton Ala2Val- $\Delta$ Ile1

\begin{tabular}{|c|c|c|c|c|}
\hline Position & $\begin{array}{l}\delta_{\mathrm{C}}[\mathrm{ppm}] ; \\
\text { mult }\end{array}$ & $\begin{array}{l}\delta_{\mathrm{H}}[\mathrm{ppm}] ; \\
\text { (mult, } \mathbf{J} \text { in } \mathrm{Hz} \text { ) }\end{array}$ & HМBC $^{\text {a }}$ & $\operatorname{cosY}^{b}$ \\
\hline \multicolumn{5}{|l|}{ Val2 } \\
\hline Val2-1 & $176.1 ; \mathrm{C} \mathrm{q}$ & & & \\
\hline Val2-2 & $70.2 ; \mathrm{CH}$ & $3.11(\mathrm{~d}, 3.6)$ & Val2-1; Val2-3; Val2-4 & Val2-3 \\
\hline Val2-3 & $31.9 ; \mathrm{CH}$ & $2.17-2.09(\mathrm{~m})$ & Val2-1 & Val2-2; Val2-4 ${ }_{\mathrm{A}} ;$ Val2-4 \\
\hline Val2- $4_{\mathrm{A}}$ & $19.3 ; \mathrm{CH}_{3}$ & $0.98(\mathrm{~d}, 6.9)$ & Val2-2; Val2-3; Val2-4 & Val2-3 \\
\hline Val2-4 & $17.5 ; \mathrm{CH}_{3}$ & $0.92(\mathrm{~d}, 6.9)$ & Val2-2; Val2-3; Val2-4 & Val2-3 \\
\hline \multicolumn{5}{|l|}{ Dha3 } \\
\hline Dha3-1 & 163.3; C q & & & \\
\hline Dha3-2 & $135.4 ; \mathrm{C} \mathrm{q}$ & & & \\
\hline \multirow[t]{2}{*}{ Dha3-3 } & $115.6 ; \mathrm{CH}_{2}$ & $\mathrm{H}_{\mathrm{A}}: 5.81($ br s) & & \\
\hline & & $\mathrm{H}_{\mathrm{B}}: 5.26(\mathrm{br} \mathrm{s})$ & Dha3-1 & \\
\hline \multicolumn{5}{|l|}{ Ala4 } \\
\hline Ala4-1 & $172.5 ; \mathrm{C} \mathrm{q}$ & & & \\
\hline Ala4-2 & $49.4 ; \mathrm{CH}$ & $4.16(\mathrm{q}, 7.3)$ & Ala4-1; Ala4-3 & Ala4-3 \\
\hline Ala4-3 & $16.8 ; \mathrm{CH}_{3}$ & $0.87(\mathrm{~d}, 5.8)$ & Ala4-1; Ala4-2 & Ala4-2 \\
\hline \multicolumn{5}{|l|}{ Pip } \\
\hline Pip-2 & 159.1; C q & & & \\
\hline \multirow[t]{2}{*}{ Pip-3 } & $23.0 ; \mathrm{CH}_{2}$ & $\mathrm{H}_{\mathrm{A}}: 3.53-3.48(\mathrm{~m})$ & & \\
\hline & & $\mathrm{H}_{\mathrm{B}}: 2.92-2.83(\mathrm{~m})$ & & Pip-4- $\mathrm{H}_{\mathrm{B}}$ \\
\hline \multirow[t]{2}{*}{ Pip-4 } & $29.4 ; \mathrm{CH}_{2}$ & $\mathrm{H}_{\mathrm{A}}: 3.88-3.81(\mathrm{~m})$ & & \\
\hline & 等, & $\mathrm{H}_{\mathrm{B}}: 2.47-2.42(\mathrm{~m})$ & Pip-2; Pip-3; Pip-5; Pip-6 & Pip-3- $\mathrm{H}_{\mathrm{B}}$ \\
\hline Pip-5 & $57.3 ; \mathrm{C} \mathrm{q}$ & & & \\
\hline Pip-6 & $63.1 ; \mathrm{CH}$ & $5.26(\mathrm{br} \mathrm{s})$ & & \\
\hline \multicolumn{5}{|l|}{ Thz6 } \\
\hline Thz6-1 & 161.0; C q & & & \\
\hline Thz6-2 & $149.7 ; \mathrm{C} \mathrm{q}$ & & & \\
\hline Thz6-3 & $127.1 ; \mathrm{CH}$ & $8.01(\mathrm{~s})$ & Thz6-2; Thz6-4 & \\
\hline Thz6-4 & $167.9 ; \mathrm{C} \mathrm{q}$ & & & \\
\hline \multicolumn{5}{|l|}{ Thr7 } \\
\hline Thr7-1 & $169.5 ; \mathrm{C} \mathrm{q}$ & & & \\
\hline Thr7-2 & $58.7 ; \mathrm{CH}$ & $4.59(\mathrm{~d}, 2.0)$ & Thz6-1; Thr7-1; Thr7-3 & Thr7-3 \\
\hline Thr7-3 & $67.1 ; \mathrm{CH}$ & $4.26-4.20(\mathrm{~m})$ & & Thr7-2; Thr7-4 \\
\hline Thr7-4 & $19.3 ; \mathrm{CH}_{3}$ & $1.13(\mathrm{~d}, 5.5)$ & Thr7-2; Thr7-3 & Thr7-3 \\
\hline \multicolumn{5}{|l|}{ Dhb8 } \\
\hline Dhb8-2 & 127.3; C q & & & \\
\hline Dhb8-3 & $135.7 ; \mathrm{CH}$ & $6.08-6.03(\mathrm{~m})$ & Dhb8-2 & Dhb8-4 \\
\hline Dhb8-4 & $19.0 ; \mathrm{CH}_{3}$ & $1.13-1.09(\mathrm{~m})$ & & \\
\hline \multicolumn{5}{|l|}{ Tzn9 } \\
\hline Tzn9-1 & 171.2; $\mathrm{C} \mathrm{q}$ & & & \\
\hline Tzn9-2 & $78.3 ; \mathrm{CH}$ & $4.92(\mathrm{dd}, 11.5,9.6)$ & Tzn9-1; Tzn9-3; Tzn9-4 & Tzn9-3- $\mathrm{H}_{\mathrm{A}} ; \mathrm{Tzn} 9-3-\mathrm{H}_{\mathrm{B}}$ \\
\hline \multirow[t]{2}{*}{ Tzn9-3 } & $36.1 ; \mathrm{CH}_{2}$ & $\mathrm{H}_{\mathrm{A}}: 3.60(\mathrm{dd}, 11.2,9.1)$ & Tzn9-1; Tzn9-2; Tzn9-4 & Tzn9-2; Tzn9-3- $\mathrm{H}_{\mathrm{B}}$ \\
\hline & & $\mathrm{H}_{\mathrm{B}}: 3.17-3.12(\mathrm{~m})$ & & Tzn9-2; Tzn9-3- $\mathrm{H}_{\mathrm{A}}$ \\
\hline Tzn9-4 & $169.5 ; \mathrm{C} \mathrm{q}$ & & & \\
\hline \multicolumn{5}{|l|}{ Ile10 } \\
\hline Ile10-2 & $52.8 ; \mathrm{CH}$ & $5.46(\mathrm{~s})$ & Ile10-3; Thz11-4 & \\
\hline Ile10-3 & $75.8 ; \mathrm{C} \mathrm{q}$ & & & \\
\hline Ile10-4 & $69.0 ; \mathrm{CH}$ & $3.88-3.81(\mathrm{~m})$ & & Ile10-5 \\
\hline Ile10-5 & $16.4 ; \mathrm{CH}_{3}$ & $1.17(\mathrm{~d}, 6.5)$ & Ile10-3; Ile10-4 & Ile10-4 \\
\hline Ile10-6 & $19.0 ; \mathrm{CH}_{3}$ & $1.08(\mathrm{~s})$ & Ile10-3; Ile10-4 & \\
\hline \multicolumn{5}{|l|}{ Thz11 } \\
\hline Thz11-1 & 162.6; C q & & & \\
\hline Thz11-2 & $149.7 ; \mathrm{C} \mathrm{q}$ & & & \\
\hline Thz11-3 & $121.9 ; \mathrm{CH}$ & $8.11(\mathrm{~s})$ & Thz11-1; Thz11-2; Thz11-4 & \\
\hline Thz11-4 & $168.2 ; \mathrm{C} \mathrm{q}$ & & & \\
\hline \multicolumn{5}{|l|}{ Thr12 } \\
\hline Thr12-2 & $51.3 ; \mathrm{CH}$ & $5.84-5.77(\mathrm{~m})$ & & Thr12-3 \\
\hline Thr12-3 & $69.0 ; \mathrm{CH}$ & 5.55 (pentet, 6.0 ) & Thz11-1; Thr12-2; Thr12-4; Q-1 & Thr12-2; Thr12-4 \\
\hline Thr12-4 & $13.9 ; \mathrm{CH}_{3}$ & $1.28-1.21(\mathrm{~m})$ & & Thr12-3 \\
\hline
\end{tabular}




\begin{tabular}{|c|c|c|c|c|}
\hline Position & $\begin{array}{l}\delta_{\mathrm{C}}[\mathrm{ppm}] ; \\
\text { mult }\end{array}$ & $\begin{array}{l}\delta_{\mathrm{H}}[\mathrm{ppm}] ; \\
\text { (mult, } \mathbf{J} \text { in } \mathrm{Hz})\end{array}$ & НMBC $^{\mathbf{a}}$ & $\operatorname{COSY}^{\mathrm{b}}$ \\
\hline \multicolumn{5}{|l|}{ Thz13 } \\
\hline Thz13-2 & 159.1; C q & & & \\
\hline Thz13-3 & $125.7 ; \mathrm{CH}$ & $8.01(\mathrm{~s})$ & Thz13-4 & \\
\hline Thz13-4 & 167.9; $\mathrm{C} \mathrm{q}$ & & & \\
\hline \multicolumn{5}{|l|}{ Thz15 } \\
\hline Thz15-1 & $160.3 ; \mathrm{C} \mathrm{q}$ & & & \\
\hline Thz15-2 & $148.1 ; \mathrm{C} \mathrm{q}$ & & & \\
\hline Thz15-3 & $126.1 ; \mathrm{CH}$ & $8.09(\mathrm{~s})$ & Thz15-1; Thz15-2; Thz15-4 & \\
\hline Thz15-4 & $168.2 ; \mathrm{C} \mathrm{q}$ & & & \\
\hline \multicolumn{5}{|l|}{ Dha16 } \\
\hline Dha16-1 & 161.6; C q & & & \\
\hline Dha16-2 & 133.6; C q & & & \\
\hline \multirow{2}{*}{ Dha16-3 } & 103.2; $\mathrm{CH}_{2}$ & $\mathrm{H}_{\mathrm{A}}: 6.52(\mathrm{~d}, 2.3)$ & Dha16-1; Dha16-2 & Dha16-3- $\mathrm{H}_{\mathrm{B}}$ \\
\hline & & $\mathrm{H}_{\mathrm{B}}: 5.44(\mathrm{~d}, 2.3)$ & Dha16-1; Dha16-2 & Dha16-3- $\mathrm{H}_{\mathrm{A}}$ \\
\hline \multicolumn{5}{|l|}{ Dha17 } \\
\hline Dha17-1 & $165.8 ; \mathrm{C} \mathrm{q}$ & & & \\
\hline Dha17-2 & $132.5 ; \mathrm{C} \mathrm{q}$ & & & \\
\hline \multirow[t]{2}{*}{ Dha17-3 } & $104.3 ; \mathrm{CH}_{2}$ & $\mathrm{H}_{\mathrm{A}}: 6.32(\mathrm{~d}, 1.7)$ & Dha17-1; Dha17-2 & Dha17-3- $\mathrm{H}_{\mathrm{B}}$ \\
\hline & & $\mathrm{H}_{\mathrm{B}}: 5.50(\mathrm{~d}, 1.7)$ & Dha17-1; Dha17-2 & Dha17-3- $\mathrm{H}_{\mathrm{A}}$ \\
\hline \multicolumn{5}{|l|}{$Q$} \\
\hline Q-1 & 162.6; C q & & & \\
\hline $\mathrm{Q}-2$ & 151.6; $\mathrm{C} \mathrm{q}$ & & & \\
\hline Q-3 & $117.4 ; \mathrm{CH}$ & $7.27(\mathrm{~s})$ & Q-1; Q-2; Q-4; Q-10 & \\
\hline Q-4 & $152.3 ; \mathrm{C} \mathrm{q}$ & & & \\
\hline Q-5 & 127.3; $\mathrm{C} \mathrm{q}$ & & & \\
\hline Q-6 & 121.9; $\mathrm{CH}$ & $6.83(\mathrm{dd}, 10.2,2.5)$ & Q-5; Q-8; Q-10 & Q-7; Q-8 \\
\hline Q-7 & 134.9; $\mathrm{CH}$ & $6.01(\mathrm{~m})$ & Q-5; Q-8; Q-9 & Q-6; Q-8 \\
\hline Q-8 & $62.8 ; \mathrm{CH}$ & $3.50-3.45(\mathrm{~m})$ & Q-6; Q-7; Q-9; Q-10 & Q-6; Q-7; Q-9 \\
\hline Q-9 & $73.8 ; \mathrm{CH}$ & $4.54(\mathrm{~d}, 12.3)$ & Q-8; Q-10 & Q-8 \\
\hline Q-10 & 153.8; $\mathrm{C} \mathrm{q}$ & & & \\
\hline Q-11 & $65.6 ; \mathrm{CH}$ & $4.97(\mathrm{q}, 6.5)$ & Q-2; Q-5; Q-6; Q-12 & Q-12 \\
\hline Q-12 & $23.9 ; \mathrm{CH}_{3}$ & $1.35(\mathrm{~d}, 6.6)$ & Q-4; Q-11 & Q-11 \\
\hline
\end{tabular}

${ }^{\text {a }} \mathrm{HMBC}$ correlations are from the proton to the indicated carbon.

${ }^{\mathrm{b}} \mathrm{COSY}$ correlations are from the proton to the proton attached to the indicated position. 
Figure S37. HPLC-MS analysis of a crude culture extract from S. laurentii NDS1/int-A2L.

(A) Structure of thiostrepton Ala2Leu- $\Delta$ Ile1. The expected $[\mathrm{M}+\mathrm{H}]^{+} \mathrm{m} / \mathrm{z}$ is 1593.5 and the expected $[\mathrm{M}+2 \mathrm{H}]^{2+} \mathrm{m} / \mathrm{z}$ is 797.2 .

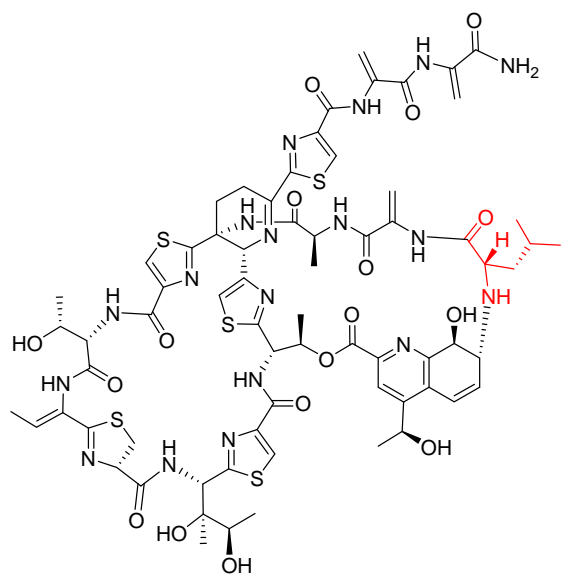

(B) Total ion chromatogram of S. laurentii NDS1/int-A2L crude extract.

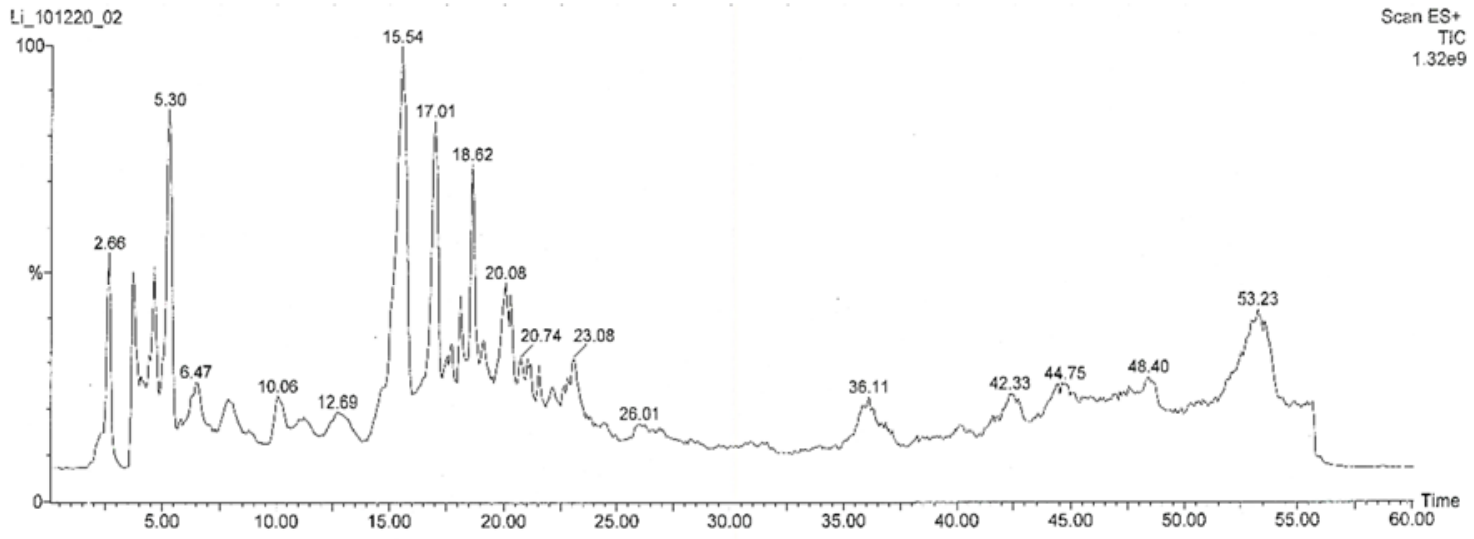

(C) Chromatogram extracted for $m / \mathrm{z} 797.2$, the calculated $[\mathrm{M}+2 \mathrm{H}]^{2+}$ ion of thiostrepton Ala2Leu$\Delta$ Ile1.

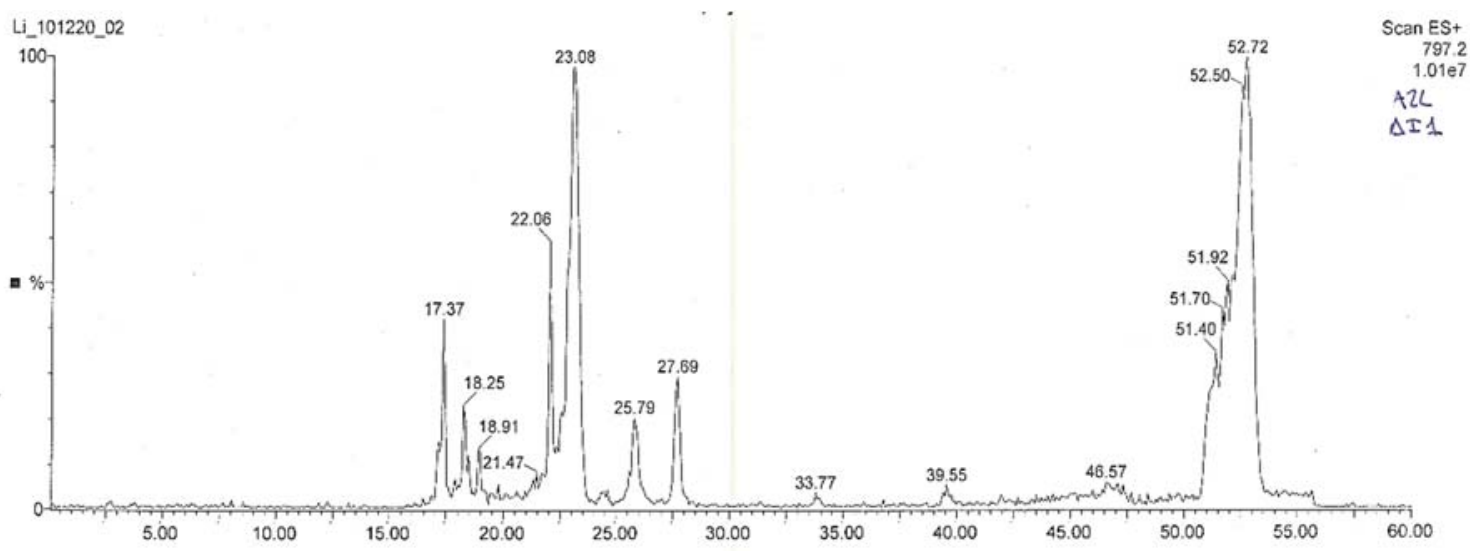


(D) Mass spectrum of the $S$. laurentii NDS1/int-A2L extract eluting at $t_{\mathrm{R}}=25.79$ minutes, which contains a +2 ion, the ionization state typically observed for thiostrepton metabolites in ESI-MS analysis, at about 797.2 and is consistent with that expected for the ring-contracted thiostrepton metabolite. Another +2 ion at about 797.2 was detected at $t_{R}$ of 22.06 minutes.

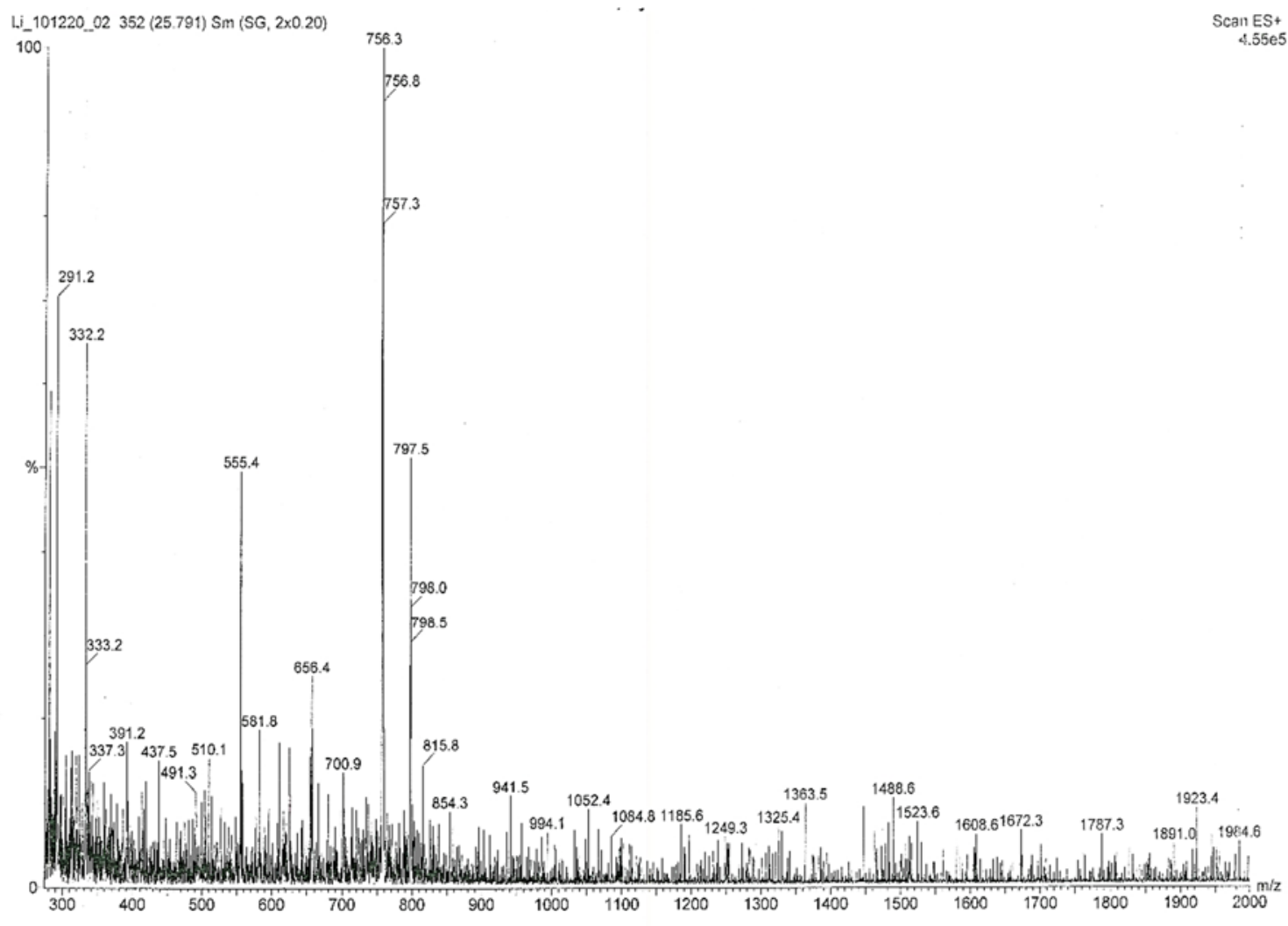


Figure S38. HPLC-MS analysis of a crude culture extract from S. laurentii NDS1/int-A2M.

(A) Structure of thiostrepton Ala2Met- $\Delta$ Ile1. The expected $[\mathrm{M}+\mathrm{H}]^{+} \mathrm{m} / \mathrm{z}$ is 1611.4 and the expected $[\mathrm{M}+2 \mathrm{H}]^{2+} \mathrm{m} / \mathrm{z}$ is 806.2 .

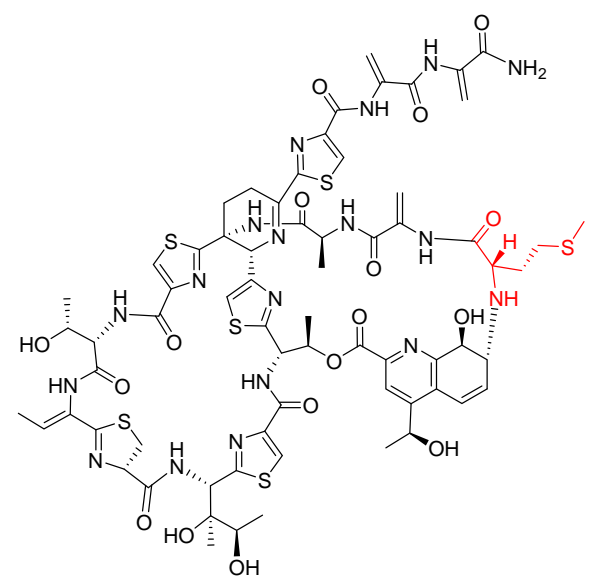

(B) Total ion chromatogram of S. laurentii NDS1/int-A2M crude extract.

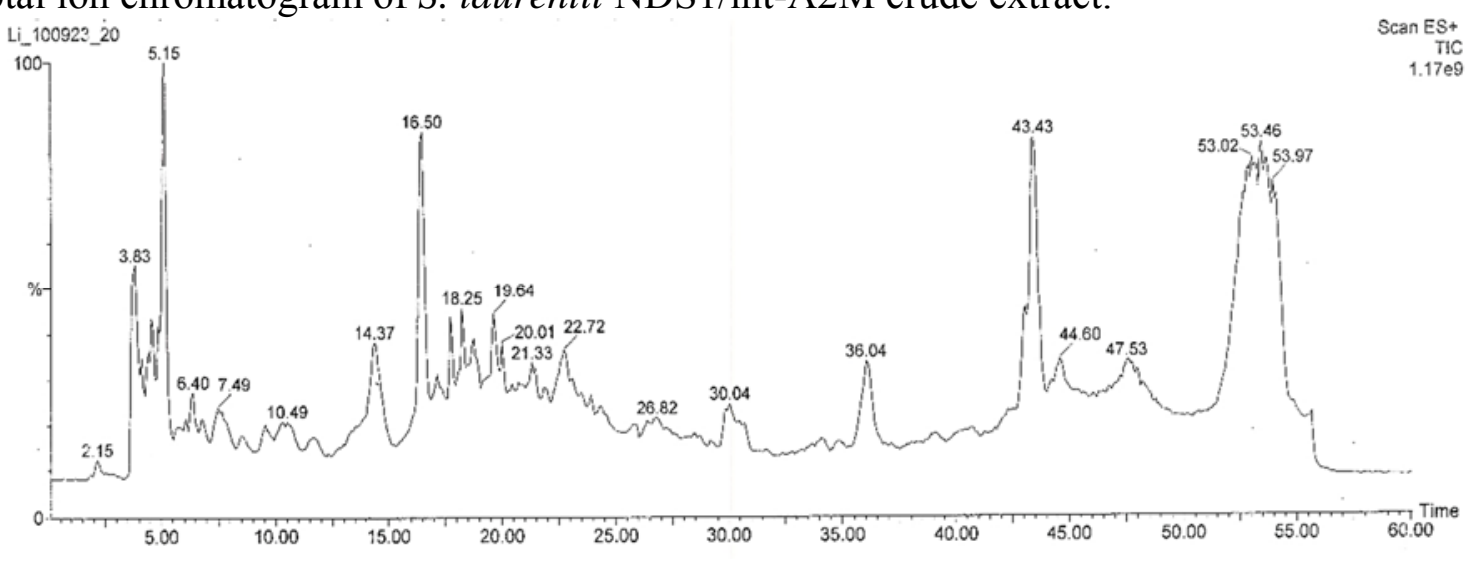

(C) Chromatogram extracted for $m / z$ 806.2, the calculated $[\mathrm{M}+2 \mathrm{H}]^{2+}$ ion of thiostrepton Ala2Met$\Delta$ Ile1.

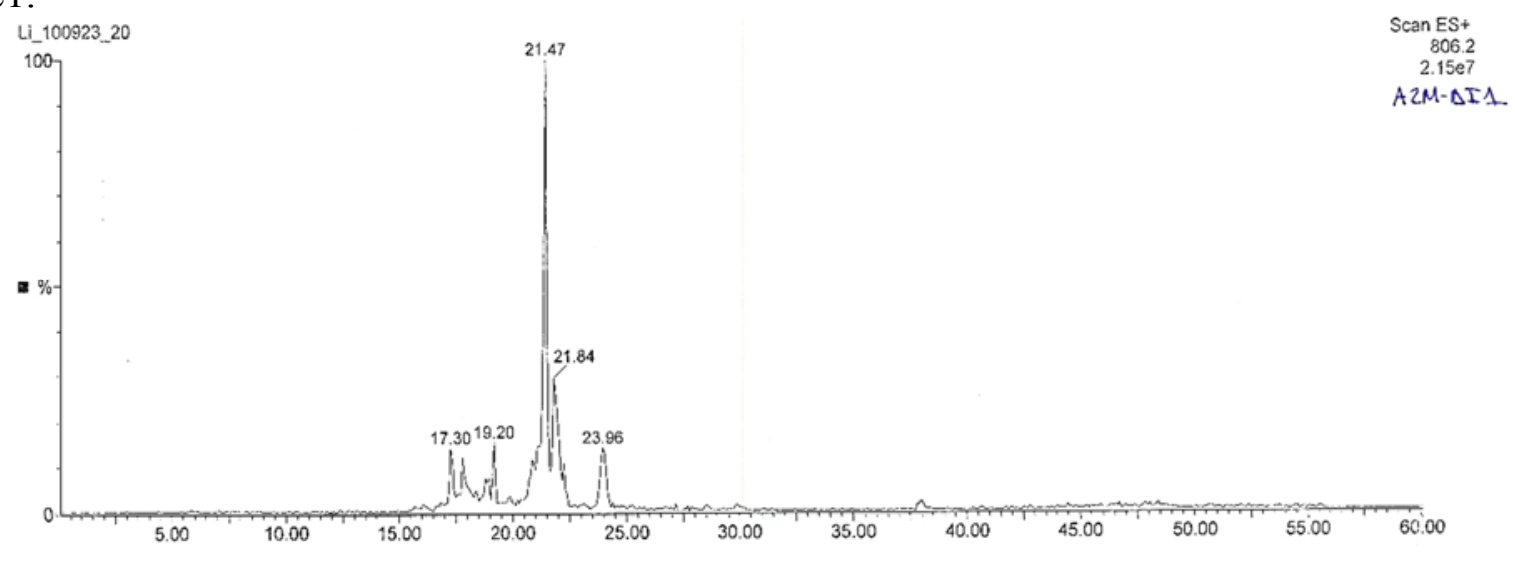


(D) Mass spectrum of the $S$. laurentii NDS1/int-A2M extract eluting at $t_{\mathrm{R}}=21.47$ minutes, which contains a +2 ion, the ionization state typically observed for thiostrepton metabolites in ESI-MS analysis, at about 806.2 and is consistent with the mass expected for the ring-contracted thiostrepton metabolite. Other +2 ions at about 806.2 were detected at $t_{\mathrm{R}}$ 's of 21.84 and 23.96 minutes.

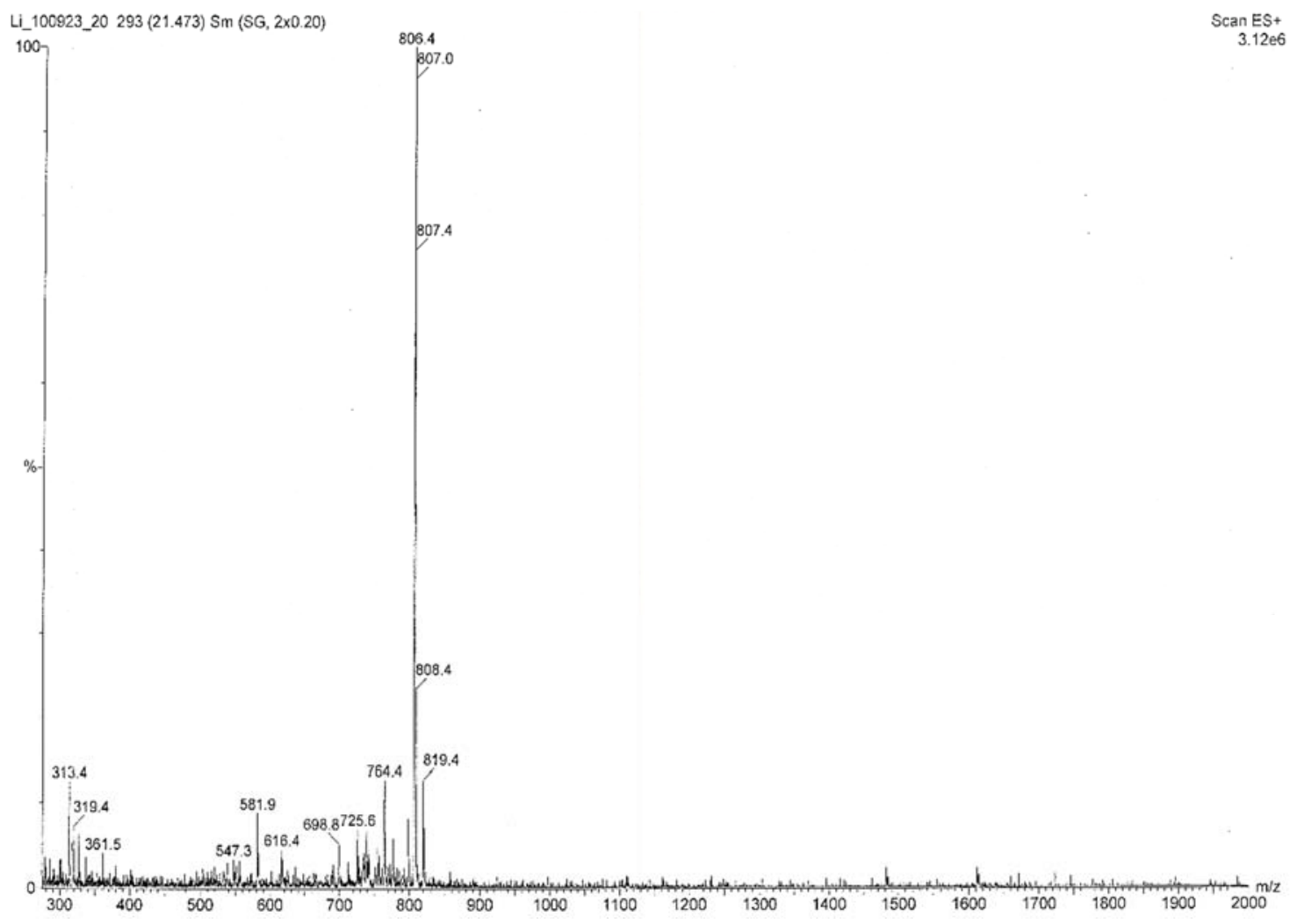


Figure S39. HPLC-MS analysis of a crude culture extract from S. laurentii NDS1/int-A2F.

(A) Structure of thiostrepton Ala2Phe- $\Delta$ Ile1. The expected $[\mathrm{M}+\mathrm{H}]^{+} \mathrm{m} / \mathrm{z}$ is 1627.4 and the expected $[\mathrm{M}+2 \mathrm{H}]^{2+} \mathrm{m} / \mathrm{z}$ is 814.2 .

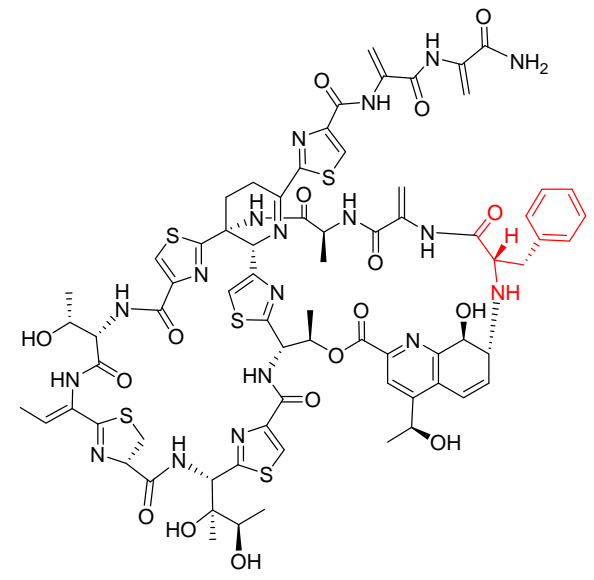

(B) Total ion chromatogram of S. laurentii NDS1/int-A2F crude extract.

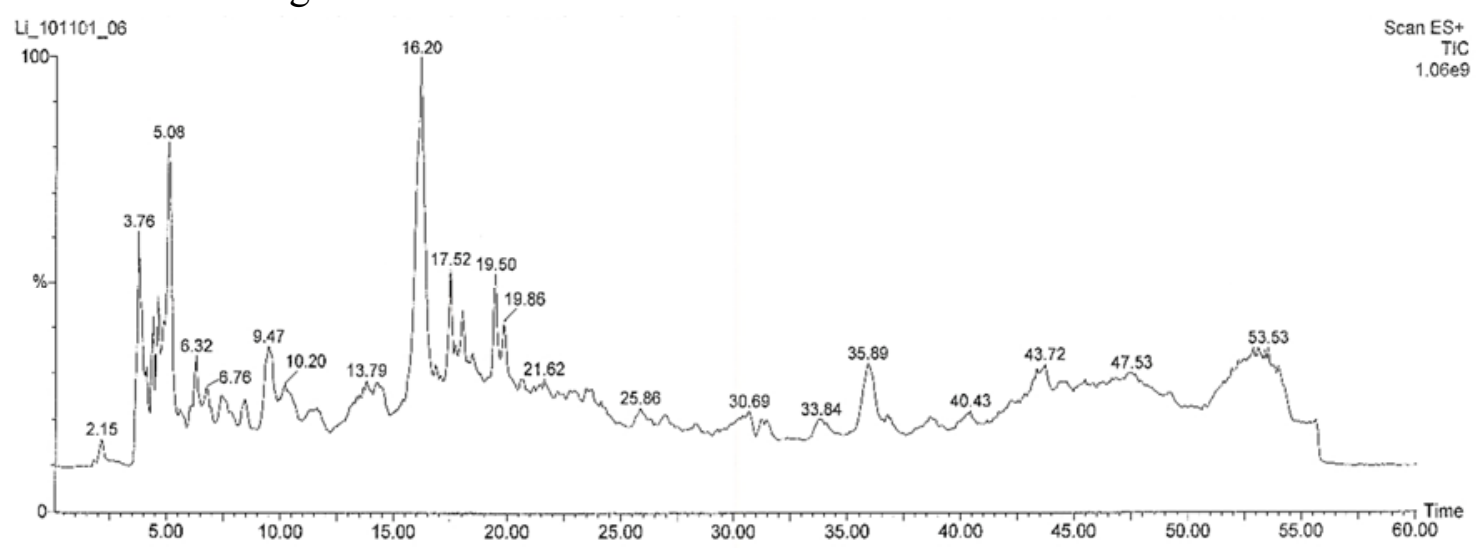

(C) Chromatogram extracted for $m / z$ 814.2, the calculated $[\mathrm{M}+2 \mathrm{H}]^{2+}$ ion of thiostrepton Ala2Phe$\Delta$ Ile1.

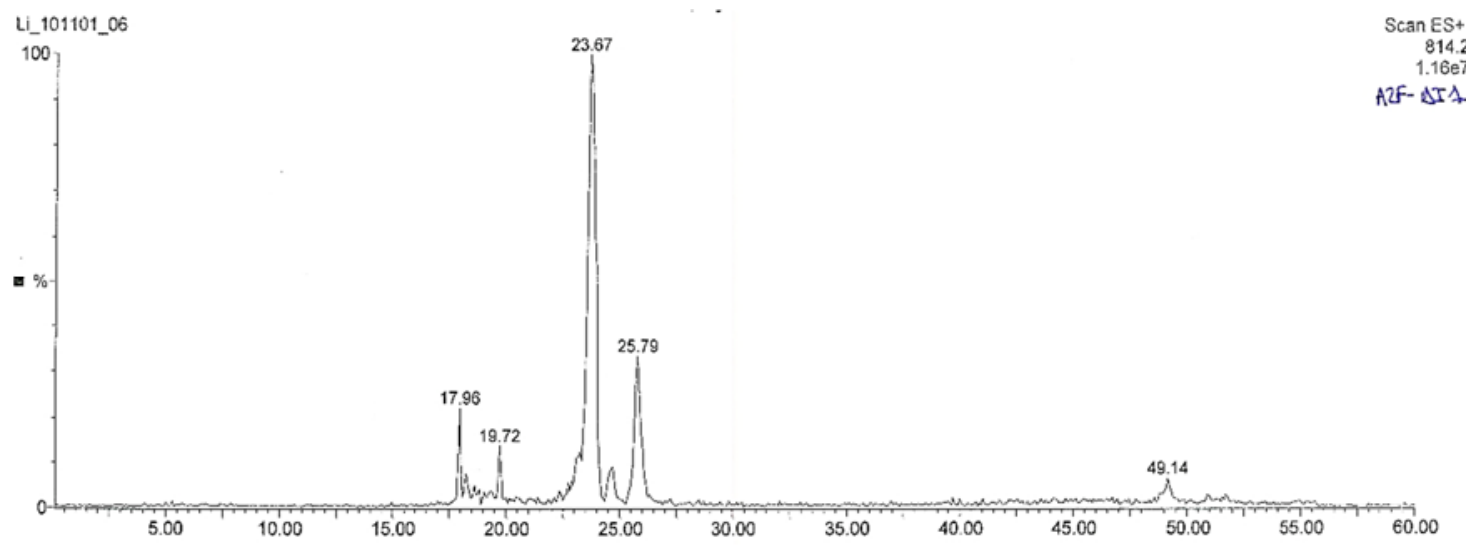


(D) Mass spectrum of the $S$. laurentii NDS1/int-A2F extract eluting at $t_{\mathrm{R}}=23.67$ minutes, which contains a +2 ion, the ionization state typically observed for thiostrepton metabolites in ESI-MS analysis, at about 814.2 and a mass consistent with that expected for the ring-contracted thiostrepton metabolite. Another +2 ion at about 814.2 was detected at a $t_{\mathrm{R}}$ of 25.79 minutes.

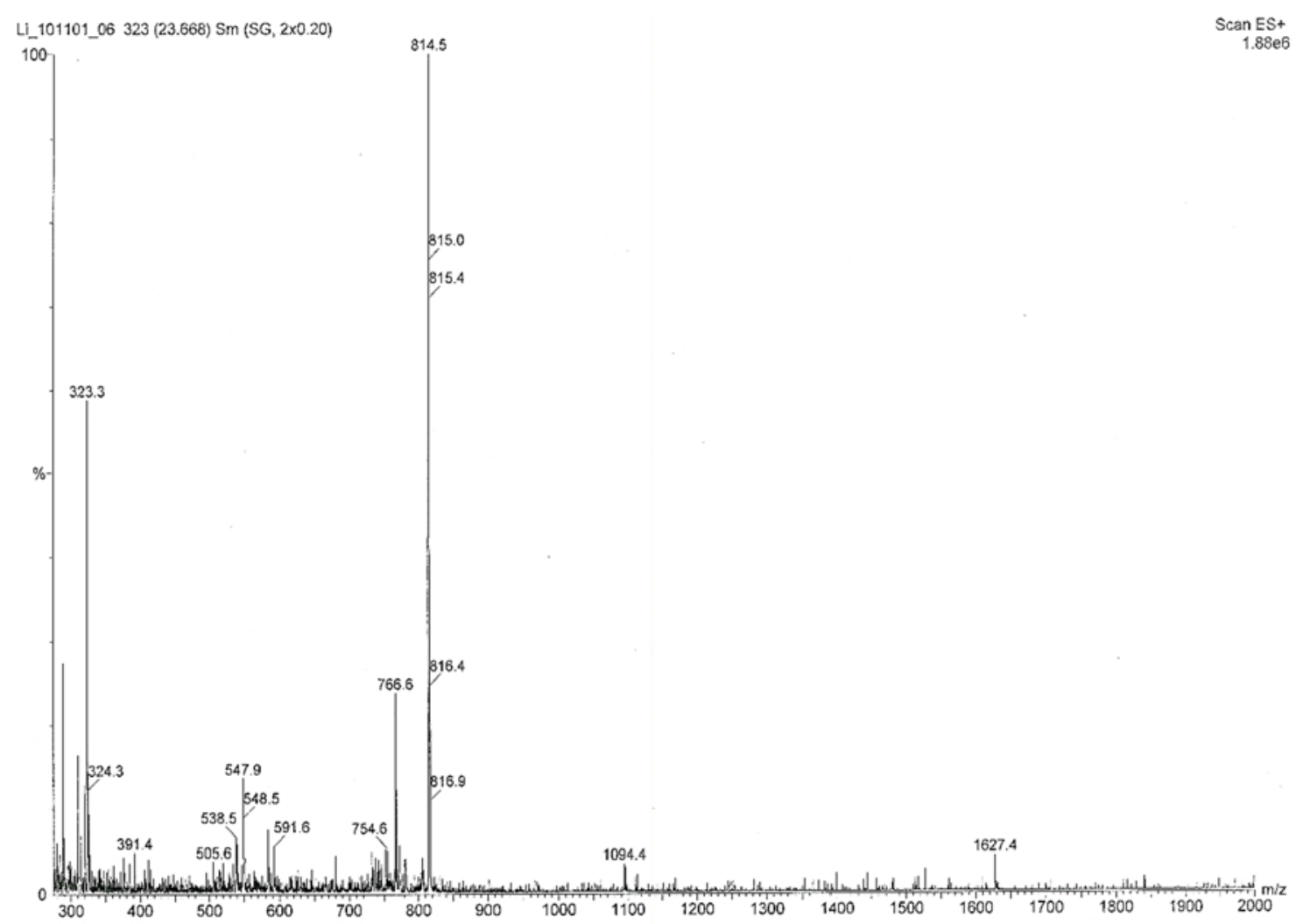


Figure S40. A representative MS analysis revealing the presence of shunt metabolites of thiostrepton biosynthesis. MS analysis of a crude culture extract from S. laurentii NDS1/int-A2H is shown.

(A) Structures of thiostrepton Ala2His and the two predicted shunt metabolites SL-1 (the wildtype analog to SL105-1 reported for the TsrA Thr7Ala variant) and SL-2 (a shunt metabolite bearing an epoxidized quinaldic acid moiety). Thiostreptons have typically been observed as the +2 ion by our HPLC-MS analysis and as the +1 ion by our MALDI-MS analysis. The expected thiostrepton Ala2His $[\mathrm{M}+2 \mathrm{H}]^{2+} \mathrm{m} / \mathrm{z}$ is 865.8 and the $[\mathrm{M}+\mathrm{H}]^{+} \mathrm{m} / \mathrm{z}$ is 1730.5 ; the expected SL-1 $[\mathrm{M}+2 \mathrm{H}]^{2+} \mathrm{m} / \mathrm{z}$ is 733.2 and $[\mathrm{M}+\mathrm{H}]^{+} \mathrm{m} / \mathrm{z}$ is 1465.4 ; and the expected SL-2 $[\mathrm{M}+2 \mathrm{H}]^{2+} \mathrm{m} / \mathrm{z}$ is 741.2 and $[\mathrm{M}+\mathrm{H}]^{+} \mathrm{m} / \mathrm{z}$ is 1481.4 .
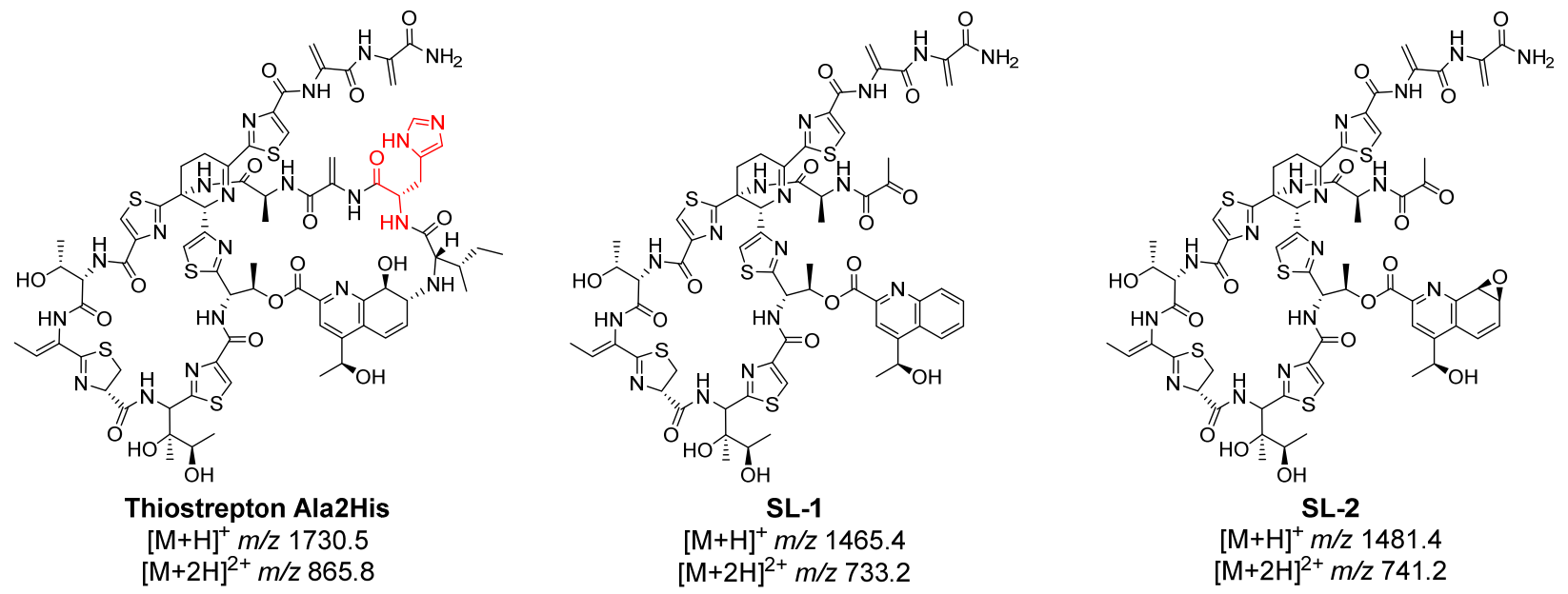

(B) Total ion chromatogram of S. laurentii NDS1/int-A2H crude extract.

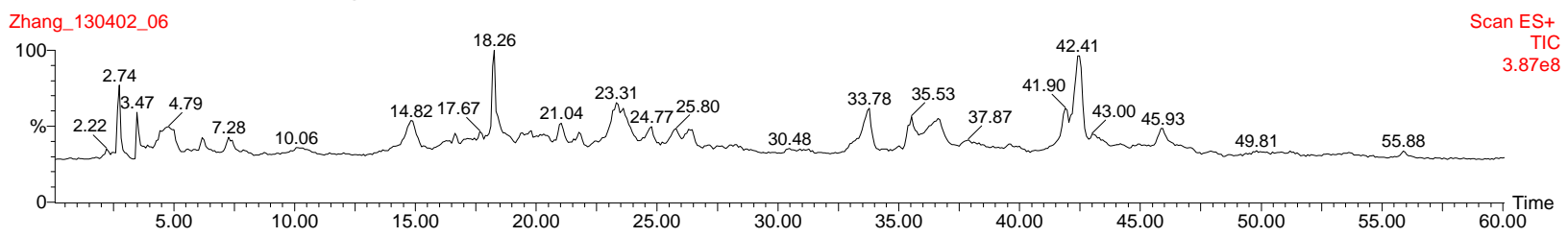

(C) Chromatogram extracted for $m / z$ 865.8, the calculated $[\mathrm{M}+2 \mathrm{H}]^{2+}$ ion of thiostrepton Ala2His.

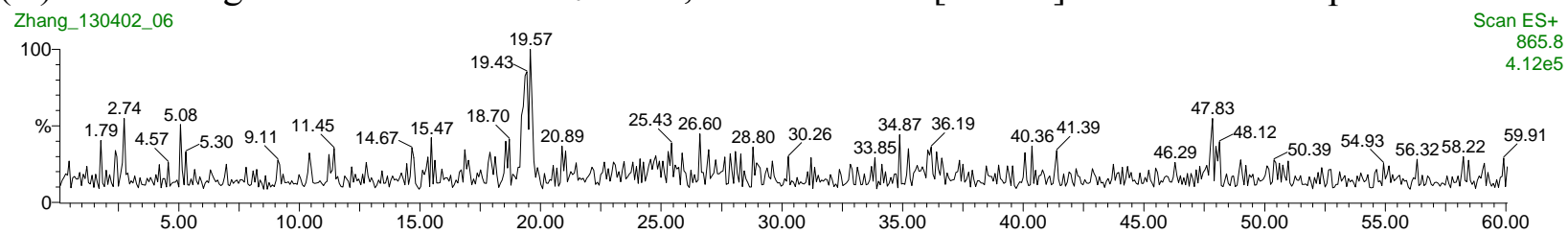

(D) Chromatogram extracted for $m / z$ 733.2, the calculated $[\mathrm{M}+2 \mathrm{H}]^{2+}$ ion of SL-1.

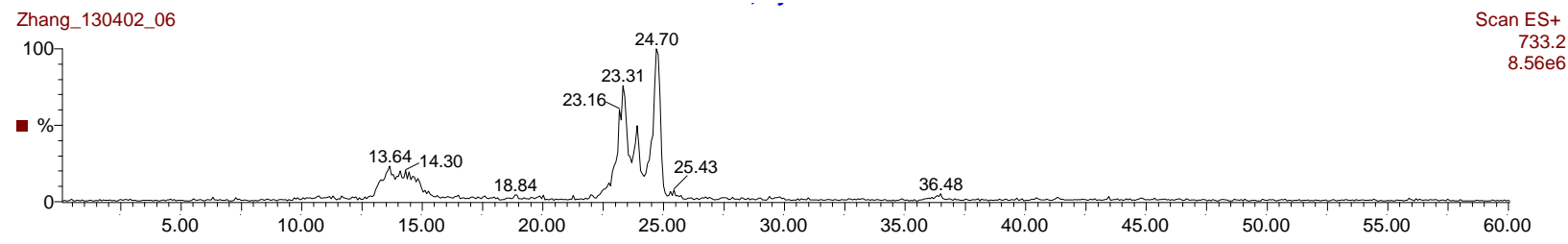


(E) Mass spectrum of the $S$. laurentii NDS1/int-A2H extract eluting at $t_{\mathrm{R}}=24.70$ minutes, which contains a +2 ion at $m / z$ 733.4, consistent with that predicted for the shunt metabolite SL-1. HRMALDI-MS analysis of partially purified SL-1 gave $[\mathrm{M}+\mathrm{H}]^{+} \mathrm{m} / \mathrm{z} 1465.3564$ (calculated 1465.3681, $-8.0 \mathrm{ppm})$.

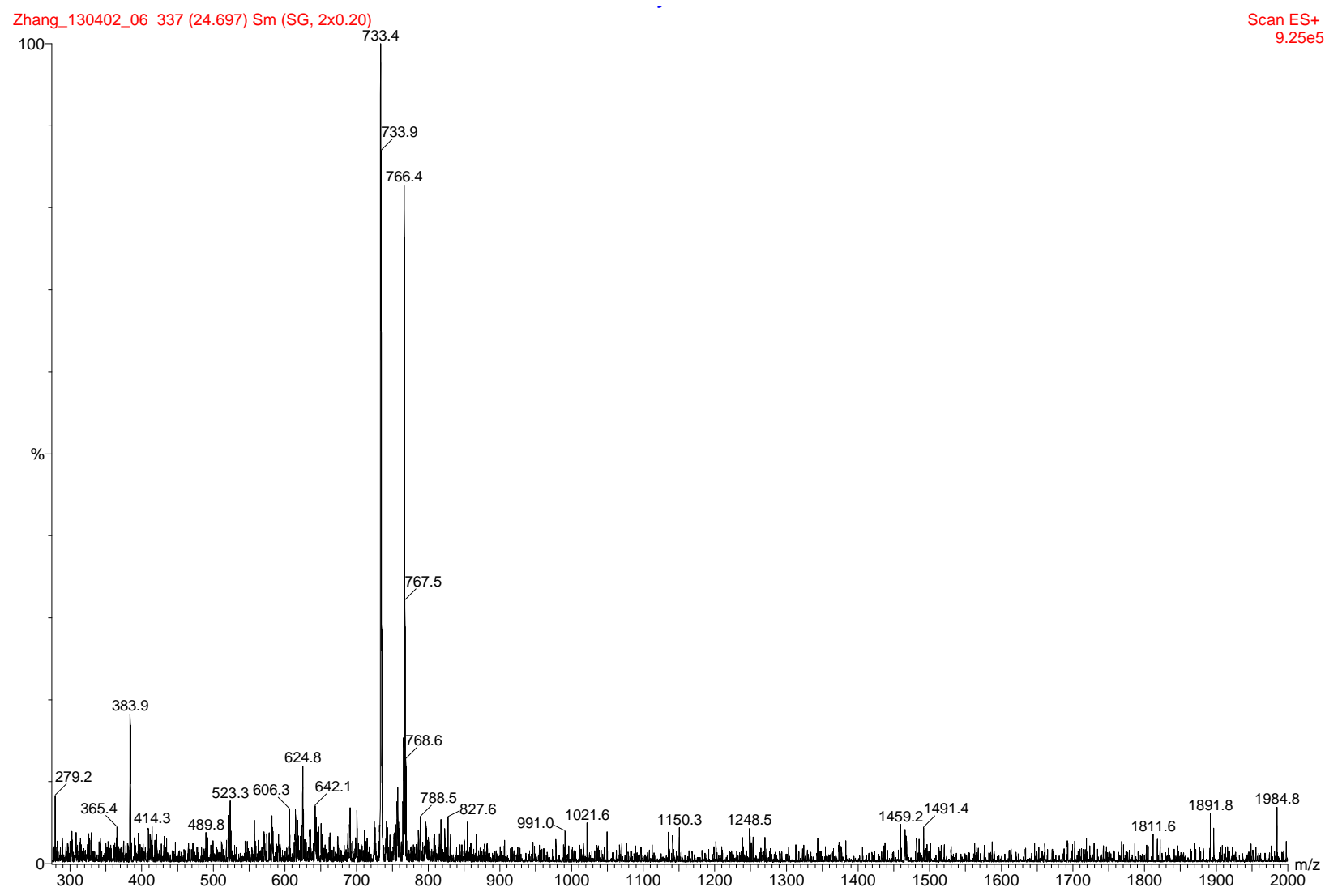

(F) Chromatogram extracted for $m / z$ 741.2, the calculated $[\mathrm{M}+2 \mathrm{H}]^{2+}$ ion of SL-2.

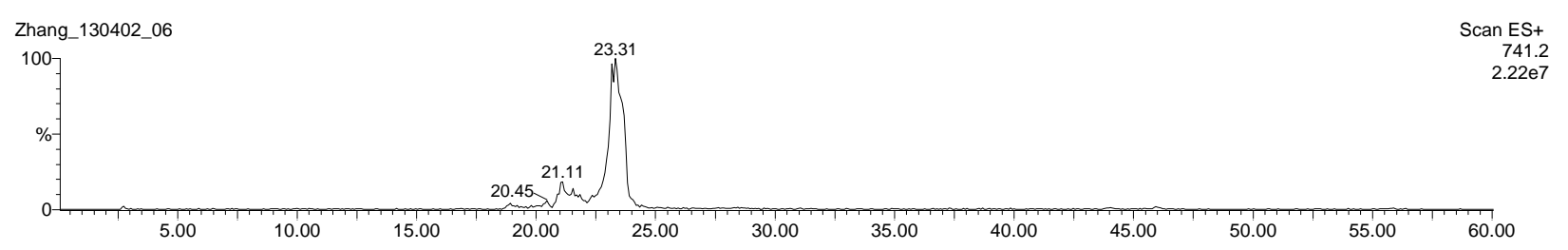


(G) Mass spectrum of the $S$. laurentii NDS1/int-A2H extract eluting at $t_{\mathrm{R}}=23.31$ minutes, which contains a +2 ion at $\mathrm{m} / \mathrm{z}$ 741.4, consistent with that predicted for the shunt metabolite SL-2. HRMALDI-MS analysis of partially purified SL-2 gave $[\mathrm{M}+\mathrm{H}]^{+} \mathrm{m} / \mathrm{z} 1481.3560$ (calculated 1481.3630, -4.7 ppm).

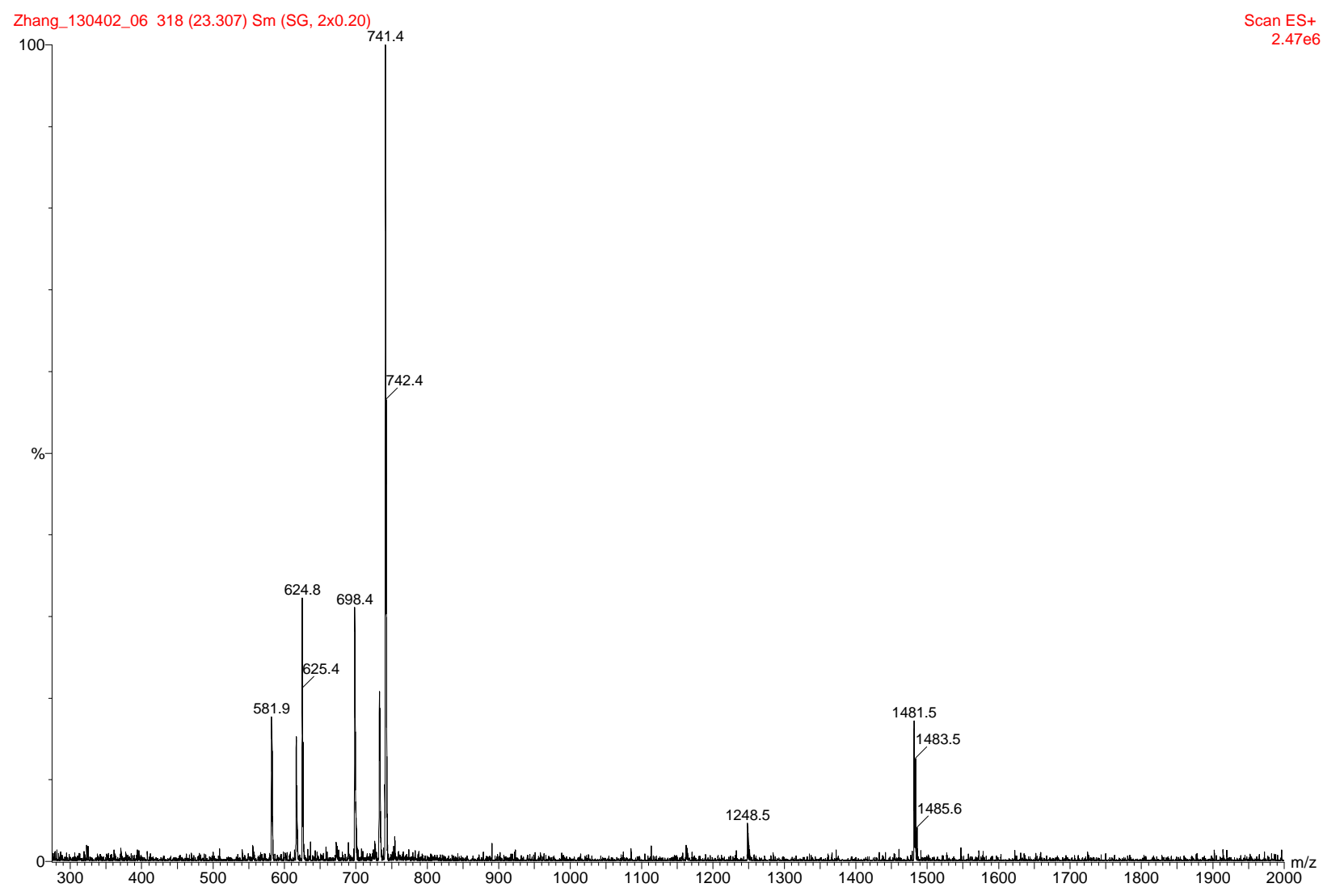


Table S5. Summary of HR-MS results of thiostrepton Ala2 analogues

\begin{tabular}{cccccc}
\hline Thiostrepton variant & Analysis & $\begin{array}{c}\text { Molecular } \\
\text { Formula }\end{array}$ & $\begin{array}{c}\text { Calculated } \\
{[\mathrm{M}+\mathrm{Na}]^{+}}\end{array}$ & $\begin{array}{c}\text { Observed } \\
{[\mathrm{M}+\mathrm{Na}]^{+}}\end{array}$ & $\begin{array}{c}\text { ppm } \\
\text { Error }\end{array}$ \\
\hline Thiostrepton Ala2Dha & HR-ESI-MS & $\mathrm{C}_{72} \mathrm{H}_{83} \mathrm{~N}_{19} \mathrm{O}_{18} \mathrm{~S}_{5}$ & 1684.4665 & 1684.4718 \\
\hline Thiostrepton variant & Analysis & $\begin{array}{c}\text { Molecular } \\
\text { formula }\end{array}$ & $\begin{array}{c}\text { Calculated } \\
{[\mathrm{M}+\mathrm{H}]^{+}}\end{array}$ & $\begin{array}{c}\text { Observed } \\
{[\mathrm{M}+\mathrm{H}]^{+}}\end{array}$ \\
\hline Thiostrepton Ala2Dhb & HR-MALDI-MS & $\mathrm{C}_{73} \mathrm{H}_{85} \mathrm{~N}_{19} \mathrm{O}_{18} \mathrm{~S}_{5}$ & 1676.5002 & 1676.5043 \\
Thiostrepton Ala2Ile- $\Delta \mathrm{Ile1}$ & HR-MALDI-MS & $\mathrm{C}_{69} \mathrm{H}_{80} \mathrm{~N}_{18} \mathrm{O}_{17} \mathrm{~S}_{5}$ & 1593.4631 & 1593.4673 \\
Thiostrepton Ala2Met & HR-MALDI-MS & $\mathrm{C}_{74} \mathrm{H}_{89} \mathrm{~N}_{19} \mathrm{O}_{18} \mathrm{~S}_{6}$ & 1724.5036 & 1724.4999 \\
Thiostrepton Ala2Phe & HR-MALDI-MS & $\mathrm{C}_{78} \mathrm{H}_{89} \mathrm{~N}_{19} \mathrm{O}_{18} \mathrm{~S}_{5}$ & 1740.5315 & 1740.5244 \\
Thiostrepton Ala2Tyr & HR-MALDI-MS & $\mathrm{C}_{78} \mathrm{H}_{89} \mathrm{~N}_{19} \mathrm{O}_{19} \mathrm{~S}_{5}$ & 1756.5264 & 1756.5267 & -2.1 \\
Thiostrepton Ala2Val- $\Delta \mathrm{Ile} 1$ & HR-MALDI-MS & $\mathrm{C}_{68} \mathrm{H}_{78} \mathrm{~N}_{18} \mathrm{O}_{17} \mathrm{~S}_{5}$ & 1579.4474 & 1579.4519 \\
\hline
\end{tabular}

Table S6. Titers of the thiostrepton Ala2 analogues

Fermentation of each S. laurentii NDS1/int-A2X variant was carried out in two sets of triplicate and crude chloroform extracts were prepared. The yields of all thiostrepton analogues were quantified by HPLC against a standard calibration curve of thiostrepton A assuming similar spectral properties for each derivative.

\begin{tabular}{cc}
\hline Compound & Titer $(\mathrm{mg} / \mathrm{L})$ \\
\hline Thiostrepton A & $115 \pm 35$ \\
Ala2Dha & $301 \pm 7$ \\
Ala2Dhb & $39 \pm 9$ \\
Ala2Gly & $19 \pm 3$ \\
Ala2Ile- $\Delta$ Ile1 & $34 \pm 4$ \\
Ala2Met & $3.1 \pm 0.2$ \\
Ala2Phe & $2.5 \pm 0.6$ \\
Ala2Tyr & $1.7 \pm 0.6$ \\
Ala2Val- $\Delta$ Ile1 & $51 \pm 16$ \\
\hline
\end{tabular}


Table S7. Aqueous solubilities of thiostrepton Ala2 analogues

The kinetic aqueous solubilities of thiostrepton $\mathrm{A}$ and its analogues were measured as described previously. ${ }^{4} 1 \mathrm{mM}$ DMSO stock solutions of thiostrepton A and its analogues were quantified by their absorbance at $280 \mathrm{~nm}$ using an extinction coefficient of $0.027 \mathrm{~cm}^{-1} \mu \mathrm{M}^{-1}$, assuming similar spectral properties for the compounds. ${ }^{6}$ The solution was diluted with buffer $(10 \mathrm{mM} \mathrm{3-}(\mathrm{N}-$ morpholino)propanesulfonic acid (MOPS), $\mathrm{pH} 7.0,5 \mathrm{mM} \mathrm{MgCl}, 200 \mathrm{mM} \mathrm{KCl}$, and 5\% DMSO) to a nominal concentration of $20 \mu \mathrm{M}$. The resulting mixture was incubated at $25^{\circ} \mathrm{C}$ for $2 \mathrm{~h}$, then the sample was centrifuged at $14,000 \mathrm{rpm}(18,000 \mathrm{x} \mathrm{g})$ at $25^{\circ} \mathrm{C}$ for $10 \mathrm{~min}$. The absorbance of the supernatant at $280 \mathrm{~nm}$ was used to define the solubility of a thiostrepton analogue. Each analysis was performed at least in triplicate.

\begin{tabular}{cc}
\hline Compound & Water solubility $(\mu \mathrm{M})$ \\
\hline Thiostrepton A & $8.10 \pm 0.24$ \\
Ala2Dha & $4.14 \pm 0.28$ \\
Ala2Dhb & $11.10 \pm 0.27$ \\
Ala2Gly & $16.79 \pm 0.60$ \\
Ala2Ile- $\Delta$ Ile1 & $10.18 \pm 0.36$ \\
Ala2Met & $13.62 \pm 0.38$ \\
Ala2Phe & $4.71 \pm 0.13$ \\
Ala2Tyr & $0.92 \pm 0.10$ \\
Ala2Val- $\Delta$ Ile1 & $3.28 \pm 0.22$ \\
\hline
\end{tabular}

Table S8. Strains and plasmids used in this study

\begin{tabular}{|c|c|c|}
\hline Strain/Plasmid & Description & Reference or source \\
\hline \multicolumn{3}{|l|}{ Streptomyces strains } \\
\hline S. laurentii ATCC 31255 & Wild-type, thiostrepton A producer & ATCC \\
\hline S. laurentii NDS1 & S. laurentii containing an in-frame deletion of tsrA & 2 \\
\hline S. laurentii NDS1/int-3A101 & S. laurentii NDS1 containing int-3A101 (A2G) & 2 \\
\hline S. laurentii NDS1/int-A2C & S. laurentii NDS1 containing int-A2C & This study \\
\hline S. laurentii NDS1/int-A2D & S. laurentii NDS1 containing int-A2D & This study \\
\hline S. laurentii NDS1/int-A2E & S. laurentii NDS1 containing int-A2E & This study \\
\hline S. laurentii NDS1/int-A2F & S. laurentii NDS1 containing int-A2F & This study \\
\hline S. laurentii NDS1/int-A2H & S. laurentii NDS1 containing int-A2H & This study \\
\hline S. laurentii NDS1/int-A2I & S. laurentii NDS1 containing int-A2I & This study \\
\hline S. laurentii NDS1/int-A2K & S. laurentii NDS1 containing int-A2K & This study \\
\hline S. laurentii NDS1/int-A2L & S. laurentii NDS1 containing int-A2L & This study \\
\hline S. laurentii NDS1/int-A2M & S. laurentii NDS1 containing int-A2M & This study \\
\hline S. laurentii NDS1/int-A2N & S. laurentii NDS1 containing int-A2N & This study \\
\hline S. laurentii NDS1/int-A2P & S. laurentii NDS1 containing int-A2P & This study \\
\hline S. laurentii NDS1/int-A2Q & S. laurentii NDS1 containing int-A2Q & This study \\
\hline S. laurentii NDS1/int-A2R & S. laurentii NDS1 containing int-A2R & This study \\
\hline S. laurentii NDS1/int-A2S & S. laurentii NDS1 containing int-A2S & This study \\
\hline S. laurentii NDS1/int-A2T & S. laurentii NDS1 containing int-A2T & This study \\
\hline S. laurentii NDS1/int-A2V & S. laurentii NDS1 containing int-A2V & This study \\
\hline S. laurentii NDS1/int-A2W & S. laurentii NDS1 containing int-A2W & This study \\
\hline S. laurentii NDS1/int-A2Y & S. laurentii NDS1 containing int-A2Y & This study \\
\hline \multicolumn{3}{|l|}{ E. coli strains } \\
\hline EPI 300 & Host for inducing high copy number of a fosmid & Epicentre $^{\circledR}$ \\
\hline BW25113/pKD46 & $\begin{array}{l}\text { Host for PCR-targeted disruption of a gene from a fosmid } \\
\text { or plasmid }\end{array}$ & $7^{1}$ \\
\hline ET12567/pUZ8002 & Host for conjugation with Streptomyces species & 1 \\
\hline \multicolumn{3}{|l|}{ Strains used for antimicrobial assays } \\
\hline Bacillus sp. ATCC 27859 & Wild-type & ATCC \\
\hline Escherichia coli ATCC 27856 & Wild-type & ATCC \\
\hline Staphylococcus aureus ATCC 10537 & Methicillin-resistant & ATCC \\
\hline Enterococcus faecium ATCC 12952 & Vancomycin-resistant & ATCC \\
\hline
\end{tabular}

Plasmids 
pSC-B-amp/kan

int-3A10

int-3A100

pCL61

int-3A101

$\mathrm{pA} 2 \mathrm{C}$

pA2D

$\mathrm{pA} 2 \mathrm{E}$

pA2F

$\mathrm{pA} 2 \mathrm{H}$

pA2I

pA2K

pA2L

pA2M

$\mathrm{pA} 2 \mathrm{~N}$

pA2P

pA2Q

pA2R

$\mathrm{pA} 2 \mathrm{~S}$

$\mathrm{pA} 2 \mathrm{~T}$

pA2V

pA2W

pA2Y

int-A2C

int-A2D

int-A2E

int-A2F

int-A2H

int-A2I

int-A2K

int-A2L

int-A2M

int-A2N

int-A2P
A routine vector from StrataClone Blunt PCR Cloning Kit, for cloning blunt-end PCR product

A fosmid containing the entire tsr gene cluster and all essential genes for the conjugal transfer and integration into a streptomycete chromosome

Derived from int-3A10, tsrA is replaced by $c h l^{R}$-sacB cassette

$\mathrm{pSC}-\mathrm{B}$-amp/kan containing the $t s r A$ variant encoding the Ala2Gly mutation

Derived from int-3A100. The $c h l^{R}$-sacB cassette is replaced by the $t s r A$ variant encoding the Ala2Gly mutation $\mathrm{pSC}-\mathrm{B}-\mathrm{amp} / \mathrm{kan}$ containing the $t s r A$ variant encoding the Ala2Cys mutation

$\mathrm{pSC}$-B-amp/kan containing the $t s r A$ variant encoding the Ala2Asp mutation

pSC-B-amp/kan containing the tsrA variant encoding the Ala2Glu mutation

pSC-B-amp/kan containing the tsrA variant encoding the Ala2Phe mutation

$\mathrm{pSC}-\mathrm{B}$-amp/kan containing the tsrA variant encoding the Ala2His mutation

$\mathrm{pSC}-\mathrm{B}-\mathrm{amp} / \mathrm{kan}$ containing the $t s r A$ variant encoding the Ala2Ile mutation

pSC-B-amp/kan containing the $t s r A$ variant encoding the Ala2Lys mutation

pSC-B-amp/kan containing the tsrA variant encoding the Ala2Leu mutation

pSC-B-amp/kan containing the tsrA variant encoding the Ala2Met mutation

$\mathrm{pSC}-\mathrm{B}-\mathrm{amp} / \mathrm{kan}$ containing the $t s r A$ variant encoding the Ala2Asn mutation

pSC-B-amp/kan containing the $t s r A$ variant encoding the Ala2Pro mutation

$\mathrm{pSC}-\mathrm{B}-\mathrm{amp} / \mathrm{kan}$ containing the $t s r A$ variant encoding the Ala2Gln mutation

$\mathrm{pSC}-\mathrm{B}-\mathrm{amp} / \mathrm{kan}$ containing the $t s r A$ variant encoding the Ala2Arg mutation

pSC-B-amp/kan containing the tsrA variant encoding the Ala2Ser mutation

pSC-B-amp/kan containing the tsrA variant encoding the Ala2Thr mutation

pSC-B-amp/kan containing the tsrA variant encoding the Ala2Val mutation

pSC-B-amp/kan containing the tsrA variant encoding the Ala2Trp mutation

pSC-B-amp/kan containing the tsrA variant encoding the Ala2Tyr mutation

Derived from int-3A100. The $c h l^{R}$-sacB cassette is replaced by the $t s r A$ variant encoding the Ala2Cys mutation

Derived from int-3A100. The $c h l^{R}$-sacB cassette is replaced by the tsrA variant encoding the Ala2Asp mutation

Derived from int-3A100. The $c h l^{R}$-sacB cassette is replaced by the $t s r A$ variant encoding the Ala2Glu mutation Derived from int-3A100. The $c h l^{R}$-sacB cassette is replaced by the $t s r A$ variant encoding the Ala2Phe mutation Derived from int-3A100. The $c h l^{R}$-sacB cassette is replaced by the tsr $A$ variant encoding the Ala2His mutation Derived from int-3A100. The $c h l^{R}$-sacB cassette is replaced by the tsrA variant encoding the Ala2Ile mutation Derived from int-3A100. The $c h l^{R}$-sacB cassette is replaced by the $t s r A$ variant encoding the Ala2Lys mutation Derived from int-3A100. The $c h l^{R}$-sacB cassette is replaced by the tsr $A$ variant encoding the Ala2Leu mutation Derived from int-3A100. The $c h l^{R}$-sacB cassette is replaced by the $t s r A$ variant encoding the Ala2Met mutation Derived from int-3A100. The $c h l^{R}$-sacB cassette is replaced by the tsrA variant encoding the Ala2Asn mutation Derived from int-3A100. The $c h l^{R}$-sacB cassette is replaced by the $t s r A$ variant encoding the Ala2Pro mutation
Agilent Technologies

2

2

This study

This study

This study

This study

This study

This study

This study

This study

This study

This study

This study

This study

This study

This study

This study

This study

This study

This study

This study

This study

This study

This study

This study

This study

This study

This study

This study

This study

This study 


$\begin{array}{ll}\text { int-A2Q } & \begin{array}{l}\text { Derived from int-3A100. The } c h l^{R}-s a c B \text { cassette is replaced } \\ \text { by the } t s r A \text { variant encoding the Ala2Gln mutation }\end{array} \\ \text { int-A2R } & \begin{array}{l}\text { Derived from int-3A100. The } c h l^{R}-s a c B \text { cassette is replaced } \\ \text { by the } t s r A \text { variant encoding the Ala2Arg mutation }\end{array} \\ \text { int-A2S } & \text { Derived from int-3A100. The } c h l^{R}-s a c B \text { cassette is replaced } \\ \text { by the } t s r A \text { variant encoding the Ala2Ser mutation } & \text { This study } \\ \text { int-A2T } & \begin{array}{l}\text { Derived from int-3A100. The } c h l^{R}-s a c B \text { cassette is replaced } \\ \text { by the } t s r A \text { variant encoding the Ala2Thr mutation }\end{array} \\ \text { int-A2V } & \begin{array}{l}\text { Derived from int-3A100. The } c h l^{R}-s a c B \text { cassette is replaced } \\ \text { by the } t s r A \text { variant encoding the Ala2Val mutation }\end{array} \\ \text { int-A2W } & \begin{array}{l}\text { Derived from int-3A100. The } c h l^{R}-s a c B \text { cassette is replaced } \\ \text { by the } t s r A \text { variant encoding the Ala2Trp mutation }\end{array} \\ \text { int-A2Y } & \begin{array}{l}\text { Derived from int-3A100. The } c h l^{R}-s a c B \text { cassette is replaced } \\ \text { by the } t s r A \text { variant encoding the Ala2Tyr mutation }\end{array}\end{array}$

Table S9. Primers used in this study

\begin{tabular}{lll}
\hline Primer & Sequence & Description \\
\hline A2X & 5'-ACTACATGGACGAGACGCTGCTCGACGGTGAGGA & $\begin{array}{l}\text { Chemically synthesized ultramer to generate } \\
\text { CCTGACCGTCACGATGATCNNSTCCGCCTCCTGCAC } \\
\text { TsrA Ala2 mutants. Underlined degenerate } \\
\text { codon encodes the amino acid at the 2 }\end{array}$ \\
& $\begin{array}{l}\text { nd } \\
\text { TAACACCCGTCTGCACCTGCAGCTGCAGCTCCTGAGG } \\
\text { CCGGACCTG-3' }\end{array}$ & \\
& position of the TsrA core peptide. \\
\hline Amp-TsrA-SP-F & 5'-GAGATCAGCGACTACATGGACGAGA-3' & $\begin{array}{l}\text { Primers used in the amplification of tsrA } \\
\text { Ala2 variants. }\end{array}$ \\
\hline SDP-TsrA-SP-R & 5'-CTGCAACGGTCAGGTCCGGCGGGCG-3' & $\begin{array}{l}\text { Primers used in PCR and DNA sequencing to } \\
\text { confirm Ala2 variant fosmids. }{ }^{2}\end{array}$ \\
SD3-R & 5'-ATCGTGTTGGGCTTGACG-3' & \\
\hline
\end{tabular}

\section{References}

1. Gust, B., Challis, G. L., Fowler, K., Kieser, T., and Chater, K. F. (2003) PCR-targeted Streptomyces gene replacement identifies a protein domain needed for biosynthesis of the sesquiterpene soil odor geosmin, Proc. Natl. Acad. Sci. U.S.A. 100, 1541-1546.

2. Li, C., Zhang, F., and Kelly, W. L. (2011) Heterologous production of thiostrepton A and biosynthetic engineering of thiostrepton analogs, Mol. BioSyst. 7, 82-90.

3. Priestley, N. D., Smith, T. M., Shipley, P. R., and Floss, H. G. (1996) Studies on the biosynthesis of thiostrepton: 4-(1-hydroxyethyl)quinoline-2-carboxylate as a free intermediate on the pathway to the quinaldic acid moiety, Bioorg. Med. Chem. 4, 1135-1147.

4. Bausch, S. L., Poliakova, E., and Draper, D. E. (2005) Interactions of the N-terminal domain of ribosomal protein L11 with thiostrepton and rRNA, J. Biol. Chem. 280, 29956-29963.

5. Zhang, F., and Kelly, W. L. (2015) Saturation mutagenesis of TsrA Ala4 unveils a highly mutable residue of thiostrepton A, ACS Chem. Biol. 10, 998-1009.

6. Ryan, P. C., Lu, M., and Draper, D. E. (1991) Recognition of the highly conserved GTPase center of $23 \mathrm{~S}$ ribosomal RNA by ribosomal protein L11 and the antibiotic thiostrepton, J. Mol. Biol. 221, 1257-1268.

7. Datsenko, K. A., and Wanner, B. L. (2000) One-step inactivation of chromosomal genes in Escherichia coli K-12 using PCR products, Proc. Natl. Acad. Sci. U.S.A. 97, 6640-6645. 